\title{
Half-a-century of gamma-ray astrophysics at the Max-Planck Institute for Extraterrestrial Physics
}

\author{
Volker Schönfelder and Jochen Greiner $^{\mathrm{a}}$ (D) \\ Max-Planck Institut für extraterrestrische Physik, Giessenbachstr. 1, 85740 Garching, Germany
}

Received 28 July 2021 / Accepted 7 October 2021 / Published online 30 October 2021

(C) The Author(s) 2021

\begin{abstract}
Gamma-ray astronomy has been one of the prime scientific research fields of the Max-Planck Institute for Extraterrestrial Physics (MPE) from its beginning. Over the years, the entire gamma-ray energy range accessible from space was explored. The purpose of this review article is to summarise the achievements of the gamma-ray group at MPE during the last 50+ years. This covers a substantial part of the general history of space-based gamma-ray astronomy, for which both, general review articles (e.g. Pinkau in Exp Astron 5: 157, 2009; Schönfelder in AN 323: 524, 2002; Trimble in AIP Conf Proc 304: 40, 1994) and a detailed tabular list of events and missions (Leonard and Gehrels in https://heasarc. gsfc.nasa.gov/docs/history, version 1.0.8, 2009), have been compiled. Here, we describe the gamma-ray activities at MPE from the beginning till the present, reviewing the tight interplay between new technological developments towards new instruments and scientific progress in understanding gamma-ray sources in the sky. This covers (i) the early development of instruments and their tests on half a dozen balloon flights, (ii) the involvement in the most important space missions at the time, i.e. ESA's COS-B satellite, NASA's Compton Gamma-ray Observatory and Fermi Space Telescope, as well as ESA's INTEGRAL observatory, (iii) the participation in several other missions such as TD-1, Solar Maximum Mission, or Ulysses, and (iv) the complementary ground-based optical instruments OPTIMA and GROND to enhance selected science topics (pulsars, gamma-ray bursts). With the gradual running-out of institutional support since 2010, gamma-ray astrophysics as a main research field has now come to an end at MPE.
\end{abstract}

\section{Introduction}

\subsection{The early history of gamma-ray astronomy prior to the first detections}

In the first half of the last century, cosmic-ray scientists had started to think about the existence of gamma radiation from the cosmos. The discovery of a so-called high-altitude radiation was made in 1912 - more than 100 years ago - by Hess [72] in Vienna and Kolhörster [92] in Berlin via ionisation rate measurements in air at different altitudes. At that time, the radiation was not called "cosmic radiation", but "high-altitude radiation". The term "cosmic rays" was introduced 15 years later [115]. At that time the radiation was supposed to consist of gamma-rays, which enter the atmosphere from outside and produce recoil electrons in the atmosphere causing the measured ionisation. But as we now know, this was not the birth hour of gamma-ray astronomy. In 1929, Bothe and Kolhörster [20] discovered the corpuscular nature of the "high-altitude radiation" by means of 2 Geiger-Müller counters in coincidence, and it was still a long way to go until the first cosmic gamma-rays could be detected.

The first attempts to place upper limits on the fraction of gamma-rays in the primary cosmic radiation had been performed with balloon and rocket experiments in the 1940s and 1950s. In particular, Bruno Rossi performed major work on cosmic-rays and initiated the rocket experiments that discovered the first extra-solar source of X-rays, Sco X-1 [49]. An excellent summary of the early cosmic ray research from its beginning to about 1960 is contained in the text book "Cosmic Rays" [130]. The real history of gamma-ray astronomy started with predictions in the 1950s: Hayakawa predicted the diffuse galactic gamma-ray emission following the decay of $\pi$-mesons from cosmicray interactions with interstellar matter, and in the same year Hutchinson predicted the interstellar gamma-ray emission from cosmic-ray electron bremsstrahlung $[69,75]$.

The explicit predictions on cosmic gamma-ray sources by Morrison [116] in 1958 were overly optimistic, but were crucial in encouraging $\gamma$-ray observations. Table 1 lists the predicted fluxes from solar flares, from the Crab nebula (in the form of nucleosynthesis lines) and from radio galaxies (assuming matter-antimatter annihilation as energy source). Fluxes from these predictions are compared with presently known fluxes from these sources. The

\footnotetext{
a e-mail: jcg@mpe.mpg.de (corresponding author)
} 
Table 1 Predictions of Morrison [116] for $\gamma$-ray fluxes of certain source types, compared to present knowledge

\begin{tabular}{llll}
\hline Source & Energy range & $\begin{array}{l}\text { Predicted flux } \\
\left.\text { (photons cm } \mathrm{s}^{-1}\right)\end{array}$ & $\begin{array}{l}\text { Measured flux } \\
\left(\mathrm{photons} \mathrm{cm}^{-2} \mathrm{~s}^{-1}\right)\end{array}$ \\
\hline Solar flares & $10-100 \mathrm{MeV}$ & 0.1 to few & $\approx 10^{-2}$ \\
& $2.223 \mathrm{MeV}$ & $1-100$ & few $10^{-1}$ \\
Crab nebula & Radioactive lines & $\approx 10^{-2}$ & $<$ few $10^{-5}$ \\
Radio Galaxies (M87, Cyg A) & $10-100 \mathrm{MeV}$ & $0.1-1$ & Cen A: $\sim 10^{-5}$ \\
& $511 \mathrm{keV}$ & $0.1-1$ & $<2.6 \times 10^{-5}$ \\
\hline
\end{tabular}

predictions on solar flares were the best: they were over-estimated by only 1-2 orders of magnitude. Those from the Crab nebular and from radio galaxies were 3-4 orders of magnitude too optimistic. As a consequence, the first experiments, e.g. the OSO-I experiment in 1962/63 [119] or the S133 experiment on the ESRO TD-1 satellite [121], had not enough sensitivity and suffered from high background. Only in 1968 the first detections were reported: the galactic plane with OSO-3 [33] and the Crab with a balloon experiment [70].

As we now know, gamma-ray fluxes from celestial objects are extremely small, and together with the technological challenges and the high (instrumental and celestial) background this made gamma-ray astronomy such a difficult research field, eventually leading to the famous quote "When one photon was a discovery, two was a spectrum, and three was the Rossi prize" [156]. Sophisticated massive and large-area instruments were an absolute must for gamma-ray observations. The development of X-ray astronomy with its order of magnitude higher source fluxes and focusing potential went much faster.

\subsection{Gamma-ray detection methods and missions}

Gamma-rays of energies $<0.1 \mathrm{TeV}$ cannot penetrate deeply into the Earth's atmosphere. Therefore, for the detection of gamma-rays $<100 \mathrm{GeV}$, one has to bring the instruments above the atmosphere, either with the help of balloons, or even better into space with satellites. Gamma-rays above $\sim 100 \mathrm{GeV}$ can be detected on the ground in large Cherenkov telescopes in which electromagnetic cascade particles (electrons/positrons) originating from those gamma-rays emit flashes of Cherenkov light from the direction of the primary gamma-ray. Since this energy range was not investigated at MPE, the following concentrates solely on air-/space-based gamma-ray studies.

In order to perform gamma-ray observations, telescopes with good angular resolution are needed. A look at the physical processes which dominate the gamma-ray range shows that below $\sim 1 \mathrm{MeV}$ the photoelectric effect dominates, and above $\sim 20 \mathrm{MeV}$ the pair production (see Fig. 1), while in between it is the Compton effect.

The first-built gamma-ray telescopes were based on the pair production process. The spark chamber technique was used to visualise the electron-positron pairs produced by high-energy $\gamma$-rays above $30-50 \mathrm{MeV}$. In these wired spark chamber experiments the direction of the gamma-ray could be determined to within a few degrees - and even better at higher energies - and this within a rather large field-of-view ( $\approx 30-40^{\circ}$ radius).

Developments to build telescopes for lower energies below the pair production domain began in the early 1970s. Figure 2 summarises the telescope principles. Directionality of a few degrees was either achieved by shielding or

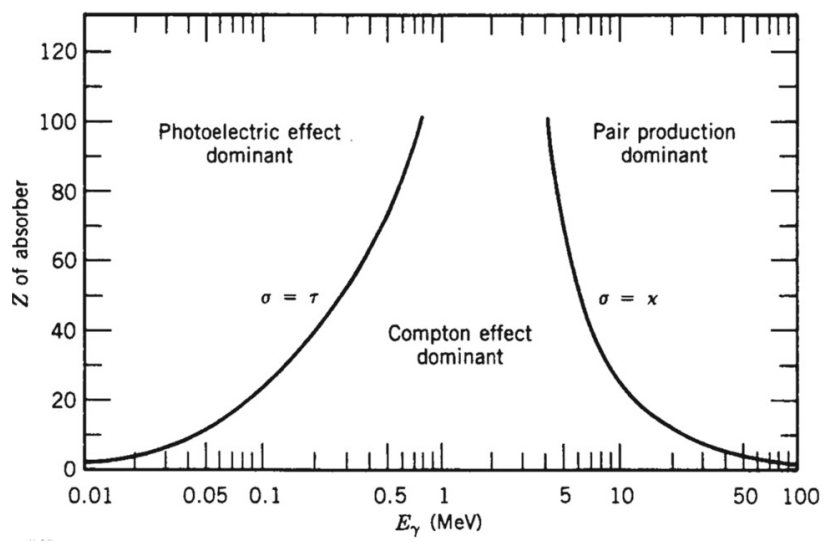

Fig. 1 Dominance of photoelectric-, Compton- and pair-production effects as a function of energy and atomic number of the interacting material 
Fig. 2 Telescope methods used in the low and medium $\gamma$-ray energy range

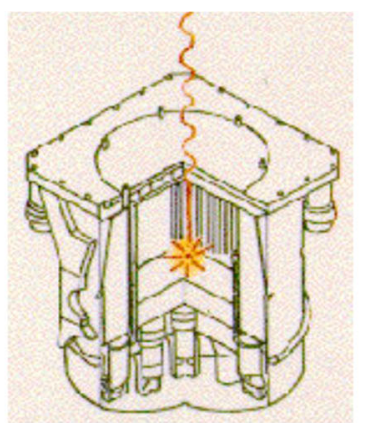

(a) Shielding/Collimation (OSSE)

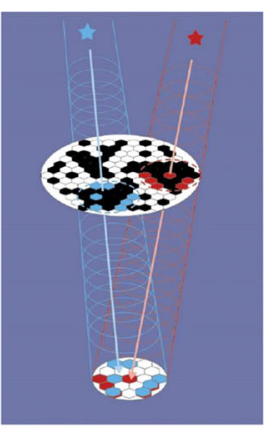

(b) Shadowing/Coded Mask (INTEGRAL)

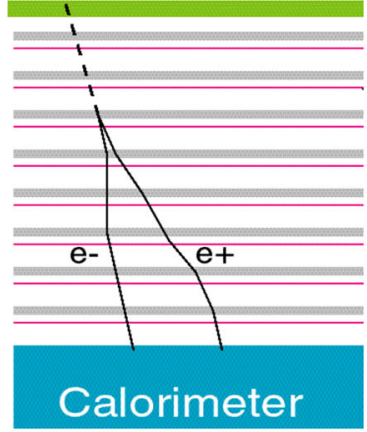

(c) Pair Creation (EGRET)

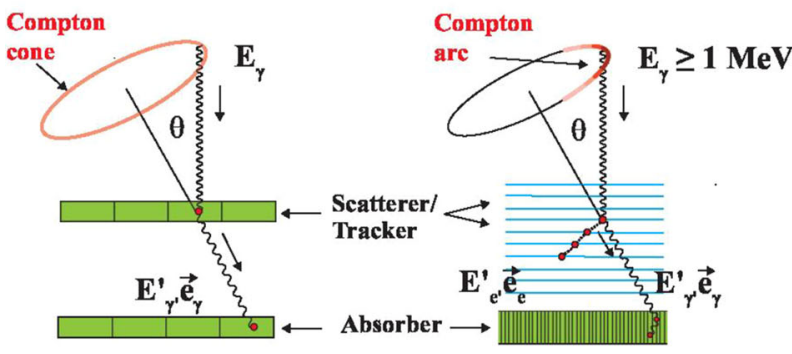

(d) Compton Imaging (COMPTEL/MEGA)

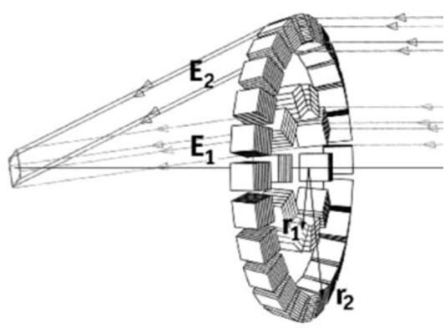

(e) Lens Concentration (CLAIRE)

shadowing with coded masks or rotation collimators. Scintillators or solid-state devices were used as detectors, working mainly in the hard X-ray range around $100 \mathrm{keV}$, up to at most $1 \mathrm{MeV}$.

In the energy range from 1 to $30 \mathrm{MeV}$ the direction of gamma-rays can be obtained with a Compton telescope. Up to the early 1970s, this energy range was considered as an impossible range for gamma-ray observations. A Compton telescope consists of 2 detector layers: the incoming gamma-ray photon undergoes Compton scattering in the upper detector, and the scattered photon is absorbed in the lower detector. From the measured energy losses in both detectors the arrival direction of the infalling gamma-ray photon can be reconstructed to a circle on the sky. Circles from many gamma-rays with different energy from the same source intersect in a single point (so-called event-circle method). Such a telescope can have a wide field-of-view of about 1 steradian and an angular resolution of a few degrees.

The history of observational gamma-ray astronomy with space missions from the early 1960s till now is illustrated in Fig. 3, concentrating on detectors operating above $100 \mathrm{keV}$. The figure does not contain all experiments (neither balloons nor satellites; a comprehensive list is maintained at https://heasarc.gsfc.nasa.gov/docs/heasarc/missions/ energy.html), but it highlights the main steps and main discoveries made over time by various experiments. The time-line can practically be subdivided into 3 sections: the first ranges from the early 1960s to about 1990 . In this period, pioneering observations of relatively small experiments with special scientific objectives were made, achieving already fascinating results. Examples are Explorer-11, the Vela satellites, OSO-3, the Apollo missions, SAS-2, OSO-7, COS-B, HEAO-C, SMM and Gamma-1.

The decade from 1991 to about 2000 marks the second section, when SIGMA/Granat, NASA's Compton GammaRay Observatory (CGRO) and BeppoSAX were in orbit. Citing Neil Gehrels, project scientist of CGRO, from his invitation letter to join the 25th anniversary celebration of the CGRO launch in 2016 (priv. comm. to VS): "The legacy of CGRO is enormous: it was the first mission performing comprehensive observations of the entire gammaray sky with broad capabilities including (1) low and high energies - the entire range of gamma-ray astronomy possible from space; (2) full sky surveys imaging the entire gamma-ray sky; (3) study of time variability - an important property of nearly all gamma-ray sources; and (4) spectroscopic studies. "This mission revolutionised our understanding of high-energy sources and processes. Its discoveries were the foundation for subsequent and current missions which mark the third section of the time-line, with the missions INTEGRAL, RHESSI, Swift, AGILE and the Fermi Observatory, several still in operation. 
Fig. 3 Summary of gamma-ray missions with instruments sensitive to photons $>100 \mathrm{keV}$, from the beginning till now. Large observatory-style missions have green text labels. [Updated from [79]]

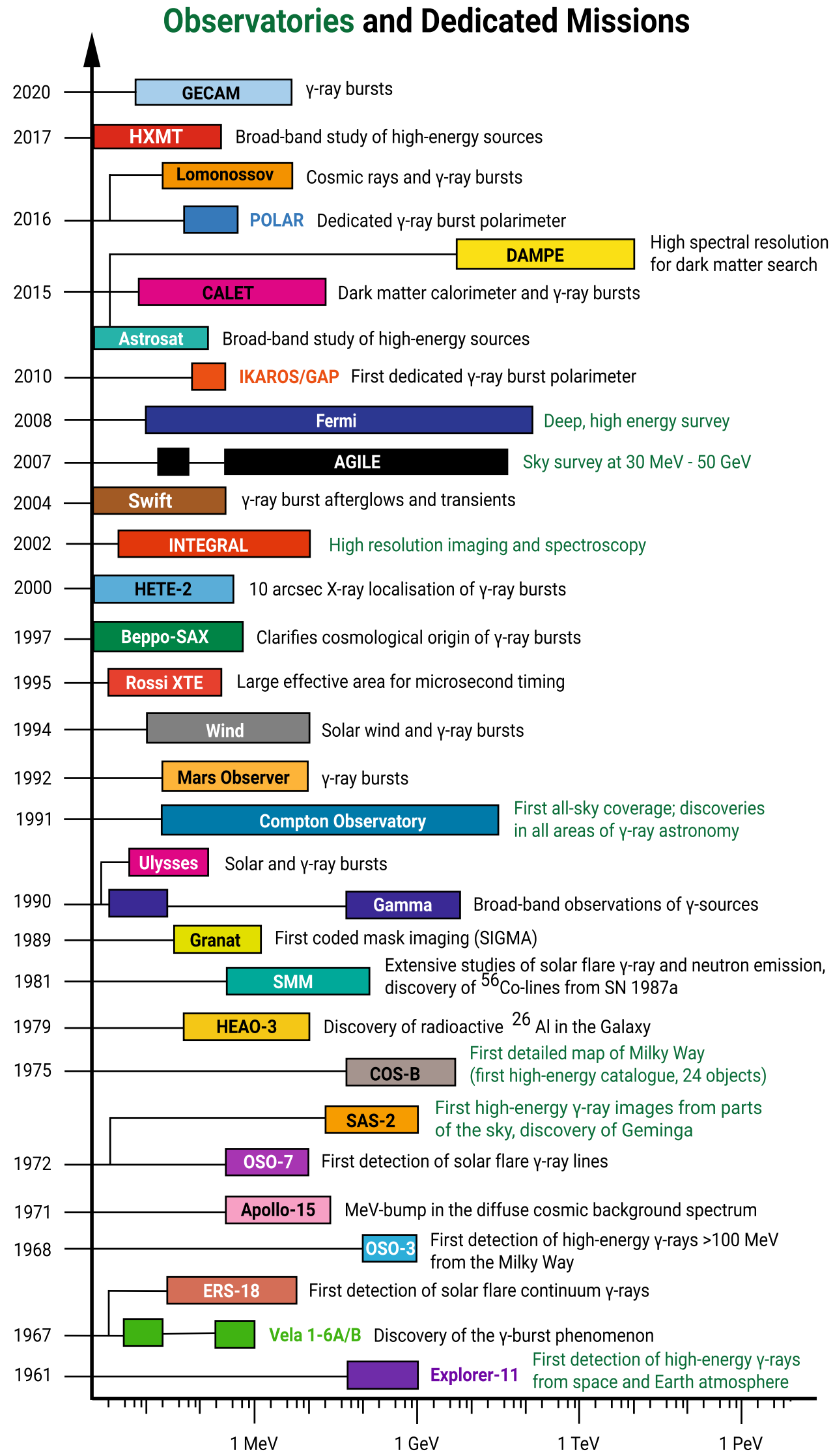




\section{Gamma-ray astronomy becomes one of the first research topics at MPE}

In 1961, only 4 years after the first satellite, Sputnik, was launched, the Max-Planck Society decided to get active in space research, and thus established a group for extraterrestrial physics at the Max-Planck Institut für Physik und Astrophysik, headed by Reimar Lüst. During a 6-month visit in the USA in 1961/1962, where he also met Bruno Rossi and Riccardo Giacconi at MIT, he studied the space research situation there. Two years later, in 1963, Lüst's group evolved into the Max-Planck Institut für extraterrestrische Physik (MPE), and Reimar Lüst was the first director. He initiated an artificial-comet experiment in the higher atmosphere to probe the solar wind. In addition, he was looking for a second research field in space science. Based on the USA experience, he also decided to build up a gamma-ray astronomy group at MPE, because this seemed to be a promising space research field with less competition than in X-ray astronomy, where substantial work had already been done (Lüst, priv. comm. to VS). The first activity was the development of a wired spark chamber. Klaus Pinkau was chosen to head that group.

Klaus Pinkau at that time was with Erich Bagge at the "Institut für Reine und Angewandte Kernphysik" in Kiel. He had been on leave from Bagges group from 1955 to 1960, working in Cecil Powell's group at the University of Bristol, where he was involved in pioneering balloon observations of cosmic rays and gamma-rays, and developed a method to determine the energy of gamma-rays by analyzing the cores of their cascade showers. After moving to Garching in 1965 he became MPE director for gamma-ray astronomy. Early members of this group were Michael Sommer, Hans Mayer (later Mayer-Hasselwander), and Hermann Rothermel. Klaus Pinkau took 5 of his students with him from Kiel to Garching: Claus Reppin, Enno Heidbreder, Volker Schönfelder, Udo Pollvogt, and Wolfgang Schmidt. For the first few years, none of these 5 joined the gamma-ray group, but instead worked on solar and high-energy neutrons as well as on cosmic-ray particles. Of those five, finally only Volker Schönfelder became a gamma-ray astronomer. The other four either joined other research groups or left the institute. The early developments resulted in balloon flights in the early 1970s.

From 1972 to 1977 Klaus Pinkau was Managing Director of MPE. In 1981 he left MPE and became director of the Max-Planck Institute for Plasmaphysics in Garching. Volker Schönfelder became his successor as head of the gamma-ray astronomy group under the directorship of Joachim Trümper (1975-2001) who was head of the X-ray group.

In the first years of gamma-ray astronomy at MPE, much of the pioneering work- especially in the field of instrument development - was achieved with balloon-borne instruments. Nearly all important discoveries, however, were made later from satellites. Balloon-borne instruments allow only relatively short observation times, and the results obtained in these flights had in most cases only marginal statistical significance, which in some cases led to wrong interpretations.

However, due to the experience MPE gained through its intensive balloon activities, it soon became very successful in the selection for satellite space missions. First highlights were the participation in the European TD-1 and COS-B missions, later the contribution to NASA's Compton Gamma-Ray Observatory with the COMPTEL and EGRET instruments, and more recently the involvement in the INTEGRAL and Fermi observatories. MPE at that time was one of the world-leading institutes in gamma-ray astronomy.

Much of the research on these missions was performed by PhD students, who later spread all over the world and stand testament to the high quality of training gained at MPE.

\section{Research in the field of gamma-ray astrophysics at MPE}

\subsection{Projects prior to 1990}

\subsubsection{The early years}

In the 1960s, gamma-ray astronomy at MPE was only performed at high energies (above 30-50 MeV). Substantial effort was directed into the development of a digital wire spark chamber. Two ambitious goals for their application were followed in parallel: The first one was to test the chambers in balloon flights in order to investigate their performance under real space conditions, and to study the diffuse cosmic and atmospheric gamma-ray components. The second one was to search for a flight opportunity on a satellite, because only then one would have sufficiently long observing time for detecting cosmic gamma-ray sources.

As to the balloon activities, altogether 3 flights were performed from Palestine (Texas) with the payload shown in Fig. 4. The spark chamber is surrounded by an anti-coincidence shield of plastic scintillator to reject charged particles. Our colleagues Mayer-Hasselwander, Pfeffermann, Pinkau, Rothermel, and Sommer were involved in these flights, later also Herterich. In these balloon flights the rate of the vertical gamma-ray flux (recognised by the direction of the electron-positron pairs) was measured as a function of the remaining atmosphere above the balloon. 


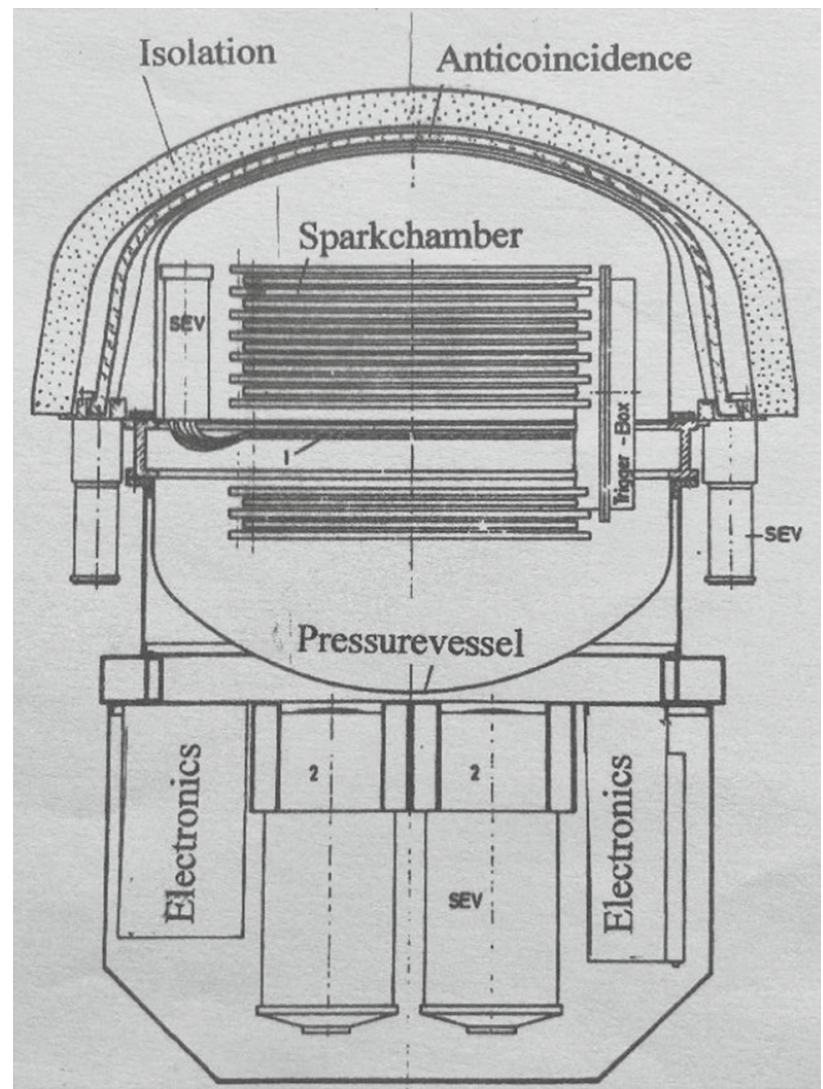

Fig. 4 MPE balloon-borne payload with the spark chamber experiment

The growth curve of the atmospheric component in the upper atmosphere can be approximated by a straight line in a double-logarithmic diagram. An excess above the extrapolation of the straight line towards the top of the atmosphere is considered to be of cosmic origin. Figure 5 shows the measured growth curve of the balloon flight on 2 June 1971 (from Mayer-Hasselwander et al. [107]). By the mid-1970s, more than a dozen of measurements by different groups were taken between $10 \mathrm{keV}$ and $100 \mathrm{MeV}$. In comparison with all the other groups, the measurements of the MPE group have to be considered as an upper limit to the real flux (see measurement point of Herterich et al. [71] in later Fig. 12).

Efforts to find a flight opportunity on a satellite were mainly undertaken by Klaus Pinkau and Reimar Lüst. As described in Pinkau [121], they had proposed a gamma-ray telescope for the German A3 mission in 1966. In the end, they asked NASA for a flight opportunity. But because NASA considered this desire as competition to the SAS-2 mission planned by Carl Fichtel and Don Kniffen, it was declined. But a first real opportunity occurred soon after. The MIMOSA collaboration consisting of Milano, Monaco (Munich), and Saclay proposed the S133 gamma-ray experiment for the ESRO TD-1 satellite. Instead of the wire spark chamber a vidicon spark chamber system was used following the request from Saclay. TD-1 was launched in 1972 and stayed in orbit for nearly 1 year. It contained two main experiments, a UV-telescope and S133. Unfortunately, this mission was not successful. The background was too high to detect gamma-ray sources (apart from one gamma-ray burst; Voges and Pinkau [163]), and finally the vidicon system failed. It was supposed that the high background was mainly produced by interactions of energetic cosmic-ray particles in the satellite and the neighbouring UV-telescope.

\subsubsection{The European COS-B satellite}

The next space gamma-ray mission in which MPE was involved, was COS-B. Already in 1967, the MIMOSA collaboration was enlarged and the Caravane collaboration was established consisting of Palermo, Milano, Saclay, MPE, Leiden, and ESTEC in Noordwijk. The wire spark chamber for COS-B (Fig. 6) was provided by MPE.

Already prior to the launch of COS-B, the existence of gamma-ray production in the Galaxy was proven by experiments in the USA, namely firstly by Explorer-11 in 1961 [94], and secondly by OSO-3 in 1968 [95]. Both were counting telescopes consisting mainly of scintillators and Cerenkov detectors, with only crude directional information (in case of OSO-3 about $25^{\circ} \mathrm{FWHM}$ ). The two experiments measured gamma-rays above 50 and 100 


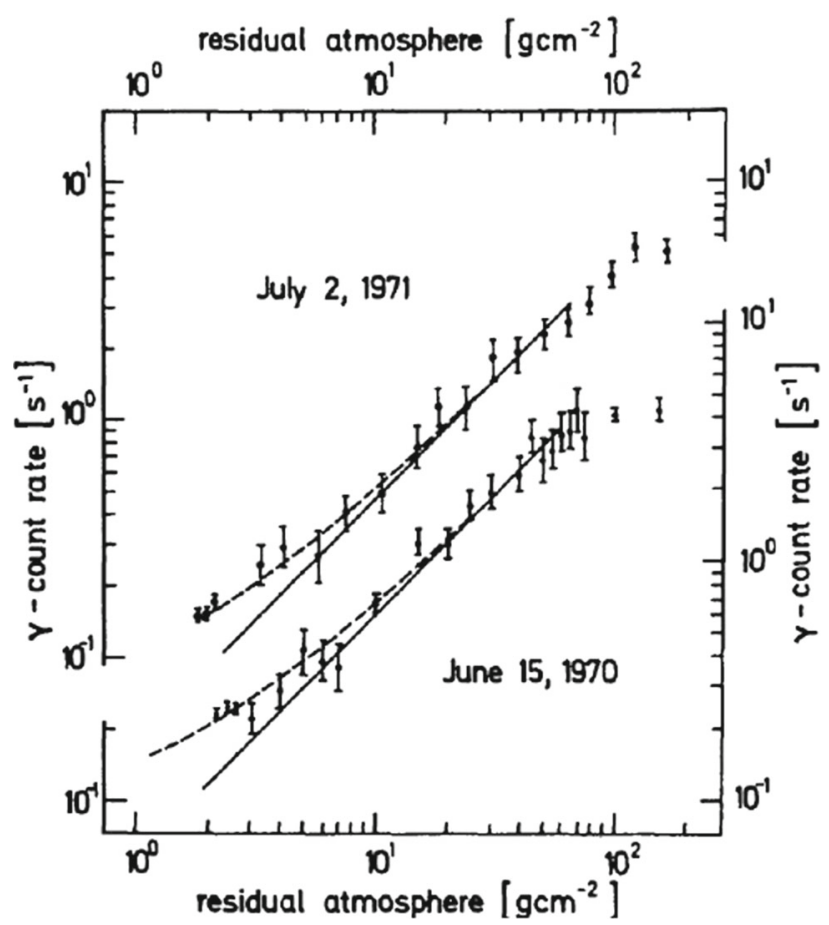

Fig. 5 Atmospheric growth curve in the $30-50 \mathrm{MeV}$ band measured by the balloon experiment of Fig. 4 during two flights [107], representing the discovery of diffuse celestial $\gamma$-ray emission: full lines mark the atmospheric component, while dashed lines show the best fit to extra-terrestrial plus atmospheric component

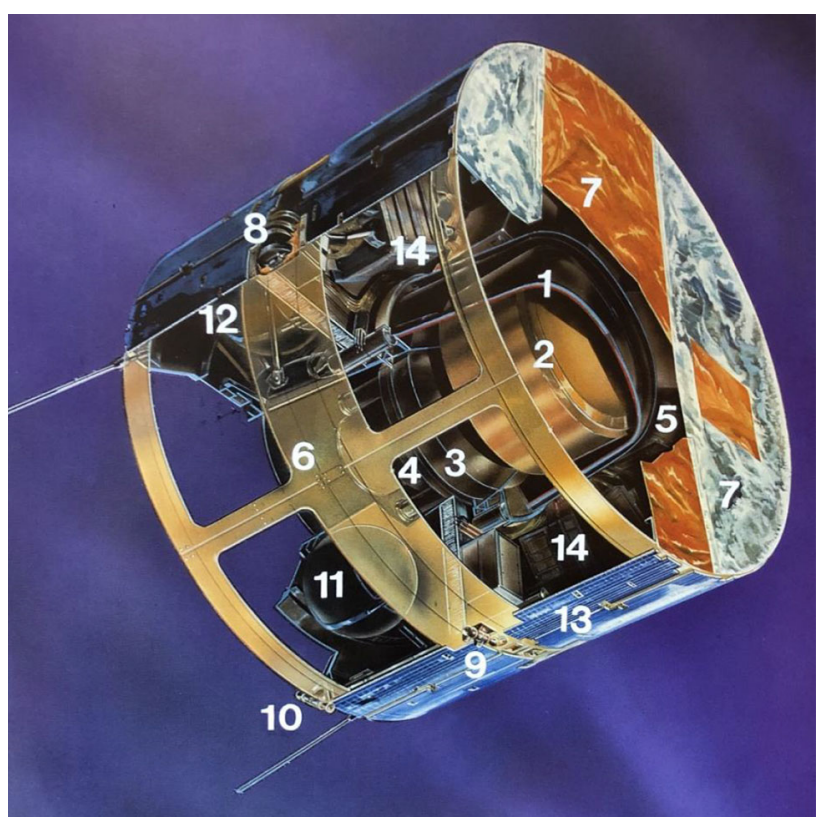

Fig. 6 Schematic of COS-B with a number of important sub-components labelled: (1) anti-coincidence counter, (2) spark chamber, (3) triggering telescope, (4) energy calorimeter, (5) experiment electronics, (6) pulsar synchroniser, (7) superinsulation, (8) Sun and Earth Albedo sensors (attitude measurements), (9) spin thruster, (10) precession thruster (attitude control), (11) nitrogen tank, (12) neon tank (gas flushing of spark chamber), (13) solar array, (14) electronics. [From Messerschmitt-Bölkow-Blohm GmbH brochure, URB 5-7-75-25 D, 1975] 


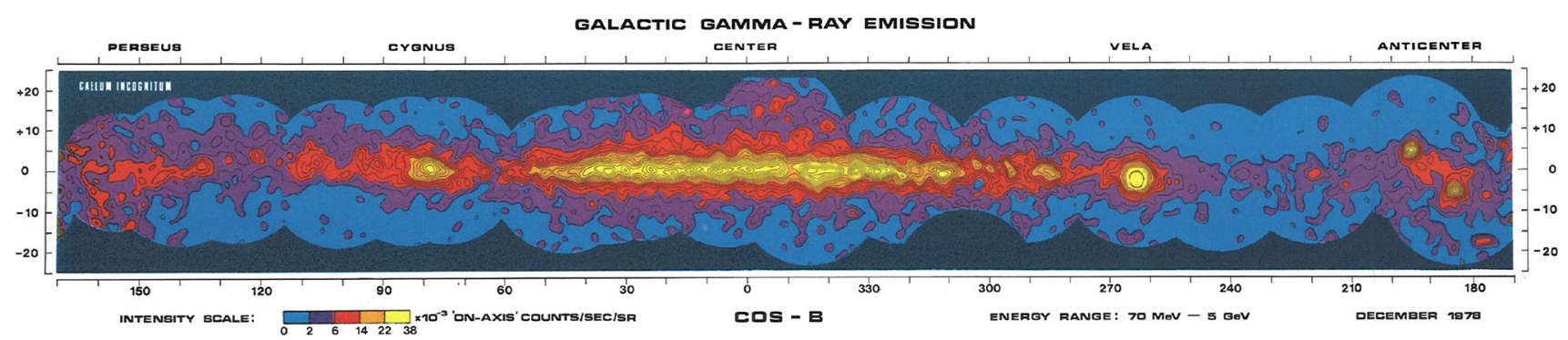

Fig. 7 Results of the complete survey of the Galactic plane by COS-B in the $70 \mathrm{MeV}$ to 5 GeV range [109]

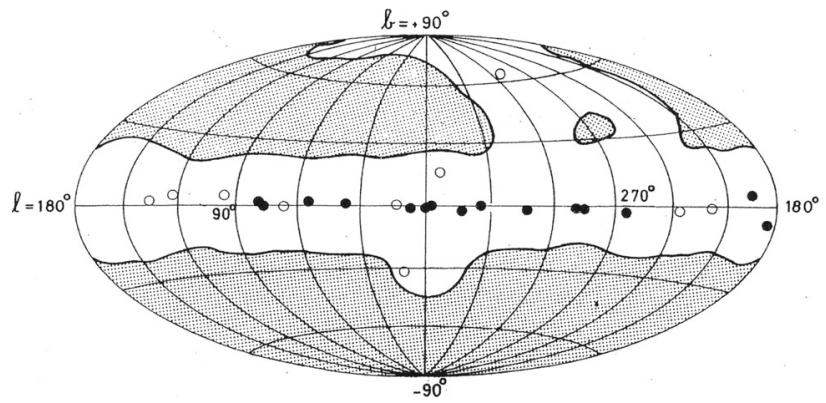

Fig. 8 Skymap of the second COS-B source catalogue [155]

$\mathrm{MeV}$, respectively. Explorer-11 detected in total 31, and OSO-3 621 cosmic gamma-rays which clearly came from the Galactic plane. The third experiment in the USA was the Small Astronomical Satellite SAS-2 [91]. It had been developed at the Goddard Space Flight Center and detected gamma-rays above $30 \mathrm{MeV}$ in a spark chamber. Launched in 1972, SAS-2 clearly saw the Milky Way and a few gamma-ray sources, especially the Crab, Vela, Cyg X-3, and a source called Geminga (which only 25 years later could be identified with ROSAT as a pulsar). Unfortunately, the mission lasted only 7 months due to a failure of the low-voltage power supply which ended the collection of data.

As ESA's first satellite, COS-B was launched in 1975 and stayed in orbit for 6.7 years. It was capable of detecting gamma-rays at energies above $\sim 50 \mathrm{MeV}$ and detected over 100.000 photons. The results were spectacular. It performed the first complete sky survey of the Galactic plane in different energy bands (see Fig. 7 for the 70 MeV-5 GeV composite from Mayer-Hasselwander et al. [109]), with surprisingly strong and fine-structured diffuse emission. While the origin of this emission from interaction of cosmic-rays with the interstellar matter had been predicted already earlier, the contradiction with the early mm-observations stirred an intense debate [109,151] which was resolved with improved $\mathrm{H}_{2}$ measurements. With the good spatial resolution, the emission of the Orion complex had been resolved earlier [30], supporting the cosmic-ray interaction. Based on spatial coincidence, another such molecular cloud, the $\rho$ Ophiuchi cloud, was identified [108]. Despite this strong diffuse emission, a number of point sources were identified, resulting in a source catalogue with 25 objects (Fig. 8 from Swanenburg et al. [155]). Except for one source, identified as the quasar 3C 273, all others were located close to the galactic plane, suggesting that 3C 273 was the only extragalactic source. Only three other sources (out of the 25) could be identified: the Crab and Vela pulsars through their timing signature (e.g. [82]), and the above-mentioned $\rho$ Ophiuchi cloud complex. It was deemed questionable that these sources were either of these two galactic source types: for molecular clouds to be visible at larger distances, the cosmic-ray flux would have been much larger than the local value, and for pulsars their $L_{\gamma} / L_{\text {radio }}$ ratio would have to be much larger than for Crab and Vela. Only later multi-wavelength (e.g. Geminga by ROSAT) and better gamma-ray observations (EGRET, Fermi/LAT) resolved this issue.

The COS-B results and its newly identified puzzles found world-wide interest in the 1970s and early 1980s among both, cosmic-ray scientists and astrophysicists. At that time, it certainly was one of the major achievements in space research in Europe. From MPE, under the lead of K. Pinkau, G. Kanbach, G. Lichti, H. Mayer-Hasselwander, M. Sommer and W. Voges were involved in the mission. In 1985, three years after the completion of the COS-B mission, its final database was handed over to the Director General of ESA in a great celebration at Palermo (Fig. $9)$.

\subsubsection{Balloons and more}

In the beginning of the 1970s, research also at lower gamma-ray energies started at MPE. Volker Schönfelder had finished his PhD in 1970 at MPE. His thesis supervisor Klaus Pinkau offered him to stay at the institute and to 


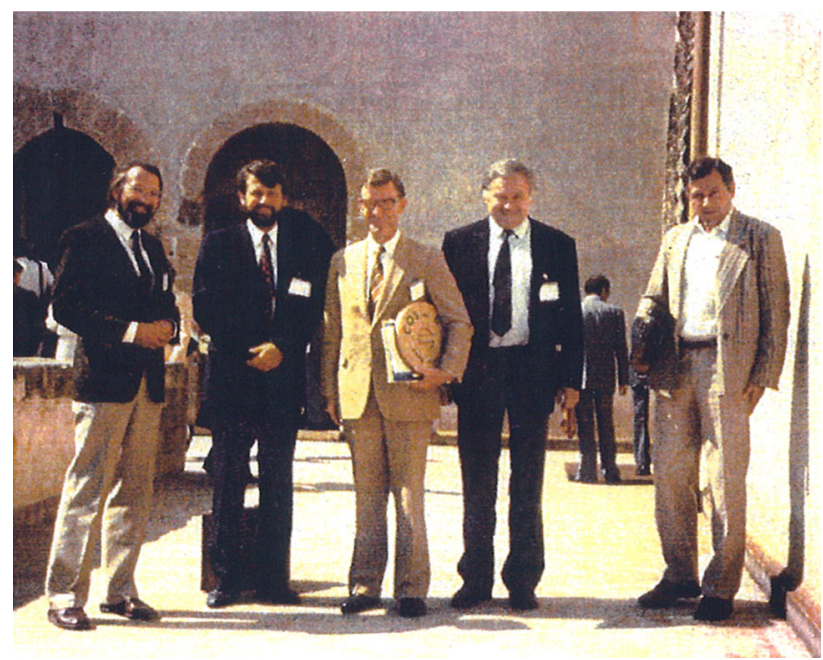

Fig. 9 This photograph with M. Sommer, W. Voges, R. Lüst, K. Pinkau, and H. Mayer-Hasselwander (from left) was taken in Palermo in 1985, when a tape with all COS-B data (the "Golden Tape") was handed over to the ESA Director General Reimar Lüst. [Photograph courtesy of G. Kanbach (MPE)]

Fig. 10 Schematics of the first MPE balloon-borne Compton telescope. In order to enhance the sensitivity, the imaging properties were not used. Instead, the analysis is restricted only to events with small scatter angles $\varphi$. Since the measured energy loss in the lower detector $\mathrm{E}_{e}^{\prime \prime}$ can be smaller than the energy of the scattered gamma-ray $\mathrm{E}_{\gamma}^{\prime}$, one can calculate a scatter angle $\bar{\varphi}$ by putting $\mathrm{E}_{\gamma}^{\prime}=$ $\mathrm{E}_{e}^{\prime \prime}$. The true scatter angle $\varphi$ is then always smaller than the calculated $\bar{\varphi}$, and by defining a maximum angle for $\bar{\varphi}$ (e.g. $\bar{\varphi}<30^{\circ}$ ), a real field-of-view of the telescope is defined

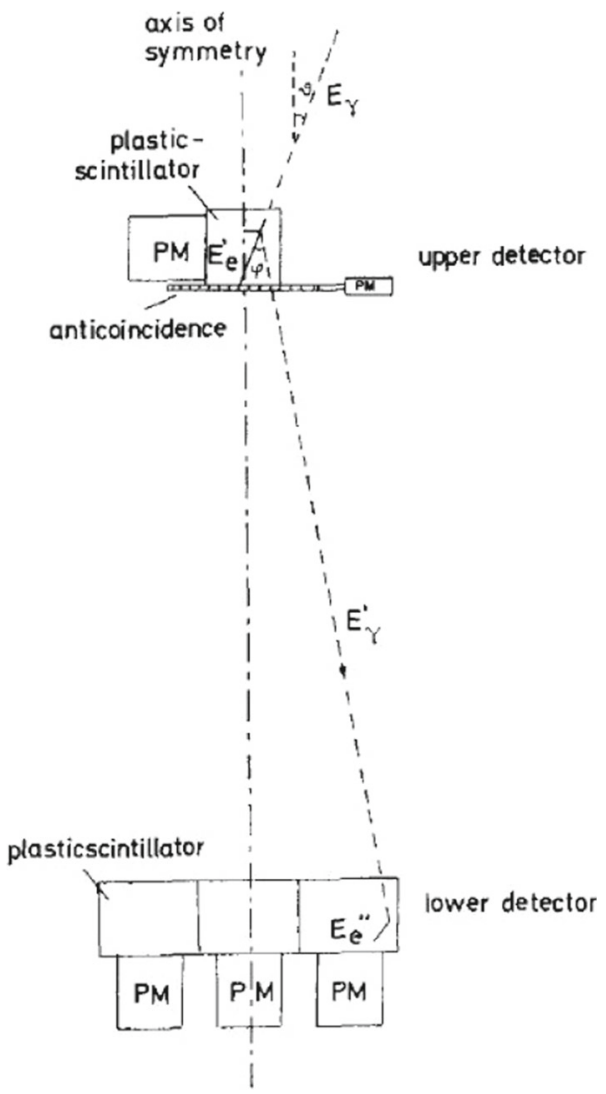

decide himself, in which area he wanted to work. A very generous offer! He thought about this offer for quite some time and then suggested to develop an astronomical telescope for the gamma-ray range between 1 and $30 \mathrm{MeV}$, which some people had called the "impossible" range for gamma-ray observations. The topic of Schönfelder's thesis had been to determine the direction and energy of high energetic cosmic-ray neutrons. The method to determine the direction of the neutrons was to let the infalling neutron scatter twice [120]. So, the idea was simple: he applied the same method to low-energy gamma-rays to determine their infalling direction, and the double-scatter Compton Telescope was born.

Two versions of such a telescope were built at MPE for balloon flights: a smaller one and a larger one which had already the size of the later COMPTEL. The schematics of the smaller one is shown in Fig. 10. It consisted of an upper detector of a $15 \times 15 \times 15 \mathrm{~cm}^{3}$ block of plastic scintillator and the lower detector (at a distance 
Fig. 11 Photograph of the telescope of Fig. 10 hanging on "Tiny Tim". Also shown is the balloon immediately after launch and at float altitude.

[Photographs courtesy of F. Schrey (MPE)]
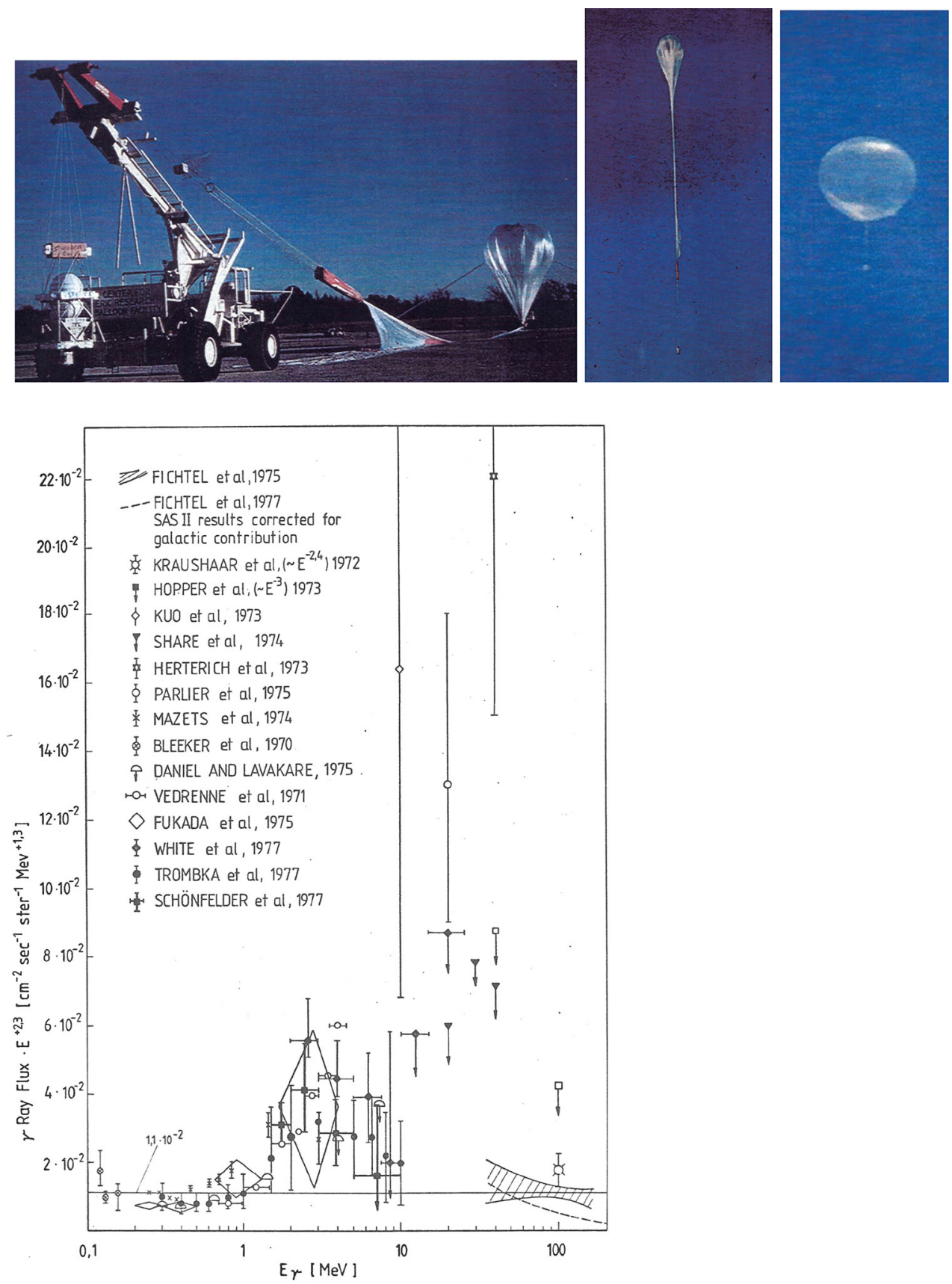

Fig. 12 Summary of measurements in the 1970s of the energy spectrum of the diffuse cosmic background radiation between $100 \mathrm{keV}$ and $200 \mathrm{MeV}$, including the measurements with the MPE balloon telescopes [71]

of $1.2 \mathrm{~m}$ ) of 9 such blocks. By means of a time-of-flight measurement, upwards scattered events were rejected. Both detectors were surrounded by a thin anticoincidence shield of plastic scintillators to reject charged particles [137]. This telescope was flown in two balloon campaigns from Palestine/Texas in 1973 and 1974 ; Fig. 11 shows photographs of the telescope hanging on the launch vehicle "Tiny Tim" just prior to launch and the balloon size immediately after launch and at float. The purpose of these flights was to measure the energy spectrum of the diffuse cosmic background radiation and to study the energy spectrum and the angular distribution of the atmospheric gamma-ray component. For the latter goal, the telescope axis could be turned around by $180^{\circ}$ in the second campaign. The telescope was not sufficiently sensitive to detect sources. Therefore, the analysis of the data was performed in such a way that only a field-of-view was defined, and no use of the imaging properties within this field was made (see Fig. 10). 


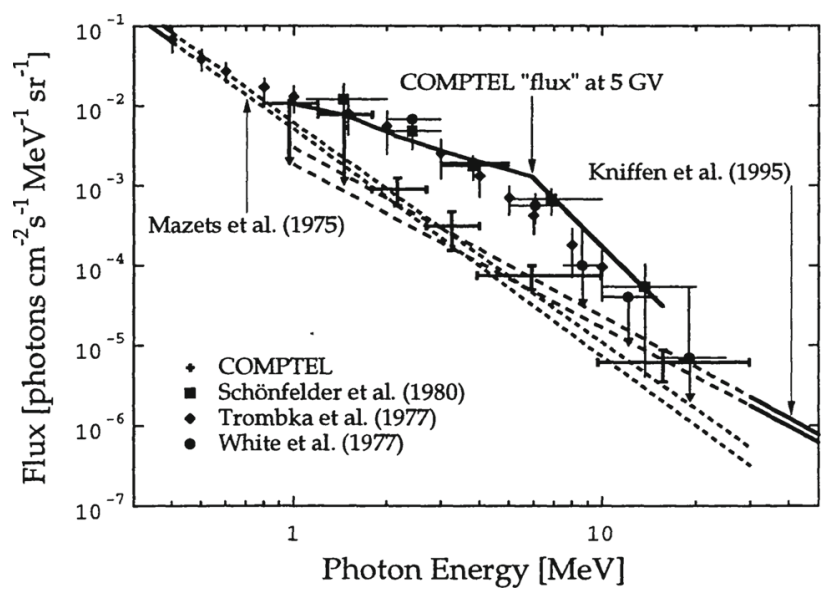

Fig. 13 COMPTEL data show that the magnitude of the MeV bump depended on the cut-off rigidity: when extrapolating to infinite cut-off rigidity, the bump vanished [90]

One of the main aims of these flights was a confirmation of the so-called MeV bump discovered by Apollo 15 [157] in the cosmic background spectrum, which considerably exceeded the interpolation from lower to higher energies. Theories about the origin of this bump were a hot topic in the 1970s, among others a nearly forgotten, but prescient explanation of the origin as cosmic-ray-induced background [46]. A compilation of the existing measurements of the energy spectrum between $100 \mathrm{keV}$ and $200 \mathrm{MeV}$ is shown in Fig. 12 [134]. The results from our balloon flights are included in this figure and confirmed the results from Apollo 15. Twenty years later, however, COMPTEL showed that the measured bump was an artefact caused by unaccounted for instrumental background radiation. COMPTEL data showed that the size of the bump was a function of the cut-off rigidity at which the observation was performed. If data at cut-off rigidity of $5 \mathrm{GeV}$ (the value at Palestine/Texas) are analysed, the Apollo-bump is reproduced. In contrast, when observations at much higher cut-off rigidities were analysed, the bump vanished completely (see Fig. 13, [90]). A detailed description of the investigations can be found in Kappadath et al. [89] and Weidenspointner et al. $[168,170]$. The Apollo 15 measurements had been performed practically at zero cut-off rigidity on the way to the Moon.

The angular resolution of atmospheric gamma-rays at $2.5 \mathrm{~g} / \mathrm{cm}^{2}$ rest atmosphere as measured in the 1974 balloon campaign showed a maximum at zenith angles of $120^{\circ}$ in the $1-3 \mathrm{MeV}$ interval. At higher energies, the maximum is shifted to lower angles, e.g. $100^{\circ}-110^{\circ}$ at $3-10 \mathrm{MeV}$ [51]. The vertical component of downward moving atmospheric gamma-rays in the 1-20 MeV range follows a power-law spectrum, which can be explained as bremsstrahlung emission of primary and secondary cosmic-ray electrons [138].

Figure 14 shows the schematics of the larger balloon telescope together with a photograph of it. It was launched from Palestine in 1977 and 1979, and from Uberaba/Brazil in 1982. The telescope consisted of 16 cells of $15 \times 15$ $\times 15 \mathrm{~cm}^{3}$ of liquid scintillator NE $213 \mathrm{~A}$ and $32 \mathrm{NaI}\left(\right.$ each $\left.15 \times 15 \times 7.5 \mathrm{~cm}^{3}\right)$ scintillators. The liquid scintillator in the upper detector was used instead of plastic scintillator in order to recognise and reject neutron-induced events by means of pulse-shape discrimination. A detailed description of the telescope is given by Schönfelder et al. [139].

The telescope was sensitive enough to detect sources. Therefore, in the analysis of the data, the imaging properties of the telescope within its field-of view were used by applying the event circle method described in chapter 1.2. In the 1977 and 1979 flights, the anticentre with the Crab nebula and its pulsar were studied [52]. In the 1982 flight (which lasted only $4.5 \mathrm{~h}$ due to a balloon failure after that time) two important results were obtained: firstly, gamma-ray emission from the radio galaxy Cen-A could be observed above $700 \mathrm{keV}$ [164], and secondly, an image in the light of the $1.809 \mathrm{MeV}$ gamma-ray line from ${ }^{26} \mathrm{Al}$ could be derived, which had its maximum brightness at $\mathrm{l}=1.4, \mathrm{~b}=-3.4$ degrees, consistent with a source at $\mathrm{l}=\mathrm{b}=0^{\circ}$ (Fig. 15 from von Ballmoos et al. [165]). It was the first image in the light of this line after its discovery by HEAO-C from the general direction of the Galactic centre region [105]. This result was the main topic of Roland Diehl's PhD thesis and determined his further scientific career. Altogether six PhD students had participated in the 5 balloon campaigns. These were Giselher Lichti, Uwe Graser, Franz Graml, Franz-Peter Penningsfeld, Peter von Ballmoos, and Roland Diehl. All six finally earned their degree with the results of these flights (e.g. Fig. 16).

With the flight in Brazil in 1982, the balloon-flight era ended. In 1977, NASA had issued the call for proposals for the planned Gamma-Ray Observatory, which later was called the Compton Gamma-Ray Observatory (CGRO). MPE was involved in 2 of the instrument proposals. For the energy range from $30 \mathrm{MeV}$ to $100 \mathrm{GeV}$, EGRET was proposed jointly by the Goddard Space Flight Center, MPE, and Stanford University. Co-Principal Investigators with equal rights were Carl Fichtel, Klaus Pinkau, and Robert Hofstadter. The second proposal was the Compton Telescope (COMPTEL) for the energy range 1-30 MeV with MPE as PI-institute, and the Laboratory for Space 
Fig. 14 Schematics (left) and a photograph (right) of the second balloon-borne Compton telescope of MPE. [From Schönfelder et al. [139]]
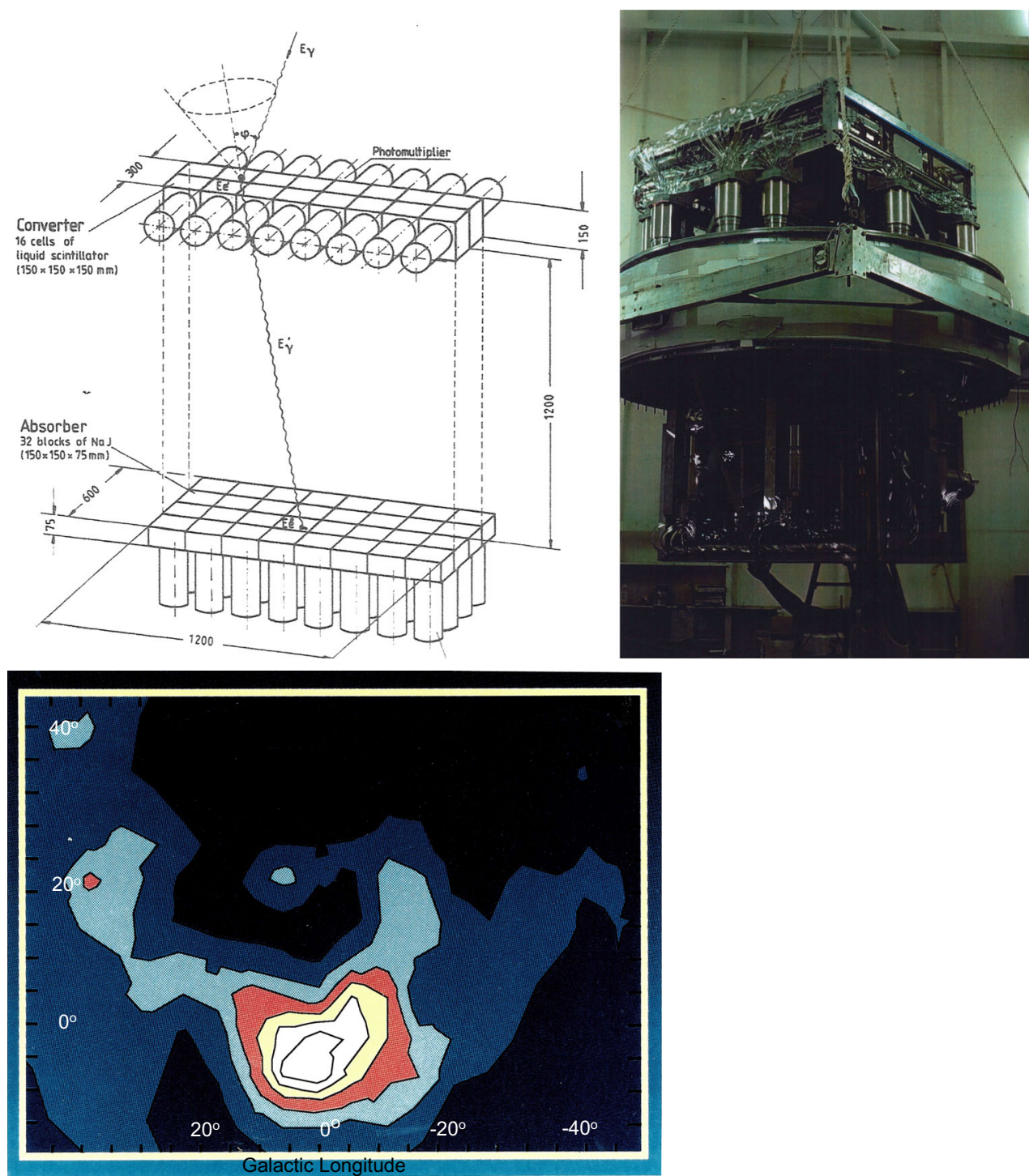

Fig. 15 Map of the Galactic centre region in the light of the $1.809 \mathrm{MeV}$ gamma-ray line from radioactive ${ }^{26} \mathrm{Al}$ as obtained by the Compton telescope flight from Uberaba in 1982 [165]

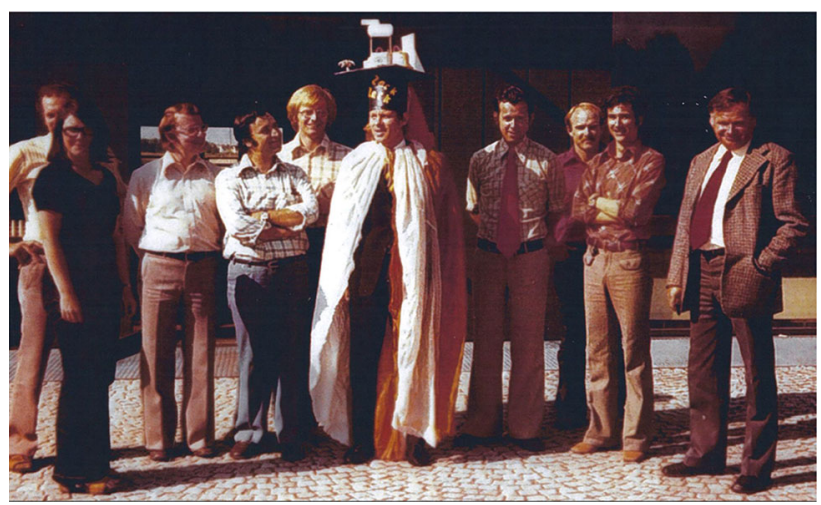

Fig. 16 Giselher Lichti in 1975 just after his PhD defence. He was Schönfelder's first PhD student. From left to right: Hönig, E. Mitchel, G. Gruner, K. Atallah, G. Kanbach, G. Lichti, V. Schönfelder, J. Daugherty, F. Graml, K. Pinkau 
Research of the University of Leiden in the Netherlands, the University of New Hampshire in the USA, and the Space Science Department of ESA in the Netherlands as Co-PI institutes. COMPTEL-PI was Volker Schönfelder, Co-PI's were Boudewijn N. Swanenburg, Jack Lockwood, and Brian G. Taylor. Both proposals were accepted by NASA in 1978 with the consequence that MPE was involved in 2 of the 4 experiments aboard CGRO, which finally became the most important gamma-ray astronomy mission of the twentieth century.

Also in 1977, a proposal (PI: Kevin Hurley, SSL Berkeley) was submitted to place GRB experiments on the twin "International Solar Polar spacecraft", one from NASA, the other from ESA. NASA cancelled its participation in 1981, leading to a 3-year launch delay, and the Challenger disaster delayed the launch another 4.5 years, until late 1990. The ESA mission was then renamed "Ulysses", due to the indirect flight path using a Jupiter fly by. Gamma-ray bursts were detected with one of the two instruments, a hard X-ray detector built by MBB and Dornier under the direction of Michael Sommer at MPE. The name of the instrument, HUS, is derived from the names HUrley and Sommer. The other instrument, a soft X-ray detector, was built by SRON. The hard X-ray detector was based on a $\mathrm{CsI}(\mathrm{Tl})$ scintillator, read out by two photomultiplier tubes (PMTs), and covered the 15-150 keV band at a sensitivity of about $10^{-7} \mathrm{erg} / \mathrm{cm}^{2} / \mathrm{s}[73]$. Ulysses has detected nearly 1800 GRBs until its deactivation in June 2009. A particularly noteworthy aspect is the (to our knowledge) first fit of the Band function [10] to a multi-instrument (Ulysses and COMPTEL) GRB spectrum [54], several years before this became the standard in the GRB community, e.g. Briggs et al. [22]. Ulysses has made significant contributions to the study of Soft Gamma Repeaters and solar flares.

But before that time, in the 1980s, the largest efforts in the MPE gamma-ray astronomy group went into the building, testing, and calibration of the two CGRO instruments, as well as into the preparation of the data analysis and the science programs. The launch of CGRO originally had been planned with one of the Space Shuttles for the mid-1980s. However, it got considerably delayed after the explosion of the Space Shuttle Challenger on 28.1.1986. Fortunately, the time till launch of CGRO in 1991 could be bridged at MPE by a more thorough analysis and interpretation of the existing balloon flight data and by a participation in the Solar Maximum Mission.

The Sun had become an interesting object for gamma-ray observations in the early 1970s. The first gamma-ray line measurements from the Sun were taken in 1972 with OSO-VII from the solar flare of August 7, 1972. Apart from the continuum emission, 4 gamma-ray lines were clearly detected: the annihilation line at 511 keV, the 2.2 $\mathrm{MeV}$ neutron capture line, and nuclear interaction lines at 4.4 and $6.1 \mathrm{MeV}$ from excited Carbon and Oxygen nuclei [32]. When the PI Ed Chupp visited MPE, he had stimulated interest in solar gamma-ray observations with some members of the gamma-ray group, and this was the reason that MPE participated in the GammaRay Spectrometer (GRS) on the Solar Maximum Mission (SMM), with Gottfried Kanbach, Klaus Pinkau, Claus Reppin, and Erich Rieger. SMM was launched in February 1980 and stayed in orbit for 9 years [47]. GRS was an array of 7 gain-controlled 7.6-cm-diameter and 7.6-cm-thick NaI-detectors. A shield around the detectors defined a field-of view of $135^{\circ} \mathrm{FWHM}$ in the solar direction. MPE provided the mechanical structure of GRS. GRS detected a wealth of solar flare gamma-ray emission in lines and continuum up to $10 \mathrm{MeV}$. A summary of all 258 flares with gamma-ray emission observed by GRS/SMM and the properties (time profiles and energy spectra) of the strongest of these is given in in Vestrand et al. [162]. A surprising discovery was the finding (with 139 flares already) that, instead of being randomly distributed in time, these flares have a tendency to occur in groups with a mean spacing of $\sim 154$ days over the 4 year observing interval [128]. This period has also been found in proton flares [8] as well as the Mt. Wilson sunspot index [9] for this time period. While correlations to the 1.3-year periodicity in the solar rotation rate [97] and others have been proposed, the Rieger period is far from understood even today.

In addition, GRS also made important studies of cosmic gamma-ray lines, e.g. of the 511 keV annihilation line and the $1.809 \mathrm{MeV}{ }^{26} \mathrm{Al}$ line, and, in particular, it succeeded in an impressive detection of nucleosynthesis lines from ${ }^{56}$ Co decay from the SN 1987a in the Large Magellanic Cloud. Likely the most curious discovery was a new type of transient in 1980 ([129], and references therein), the explanation of which was embargoed for nearly 10 years for US national security: namely its man-made origin, caused by the near-encounters (of order $600 \mathrm{~km}$ ) of the nuclear reactors believed to be carried by the Soviet Radar Ocean Reconnaissance Satellites (ROR-SATs).

\subsection{Results based on EGRET and COMPTEL onboard NASA's Compton observatory}

\subsubsection{The Compton gamma-ray observatory}

The launch of NASA's Gamma-Ray Observatory took place on 5 April 1991, and the observatory was named "Compton Gamma-Ray Observatory" (CGRO) in honour of Dr. Arthur Holly Compton, who won the Nobel prize in physics for work on scattering of high-energy photons by electrons. The Space Shuttle Atlantis brought CGRO into an orbit with an altitude of $450 \mathrm{~km}$ and an inclination of $28^{\circ}$. Prior to this event there had been numerous meetings of the PI's and the Project Scientist with technical staff members and also once with three of the five astronauts (Fig. 17). The personal climate among all these people was excellent. There had never been any dispute, and this remained so till the end of the mission. 


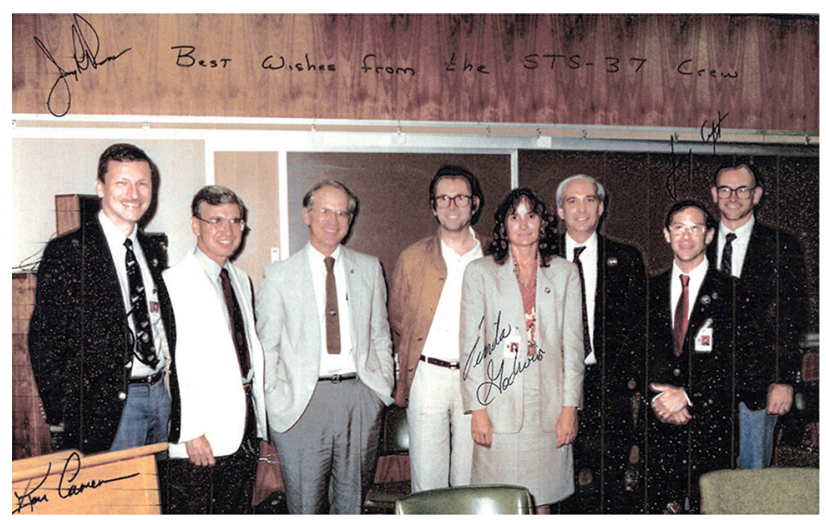

Fig. 17 The 4 principal investigators and the project scientist meet 3 of the 5 astronauts prior to launch: Shuttle captain astronaut Steve Nagel, EGRET Co-PI Carl E. Fichtel, Project Scientist Don Kniffen, COMPTEL PI Volker Schönfelder, astronaut Linda Godwin, BATSE PI Gerald J. Fishman, astronaut Jay Apt, OSSE PI Jim Kurfess (from left to right)

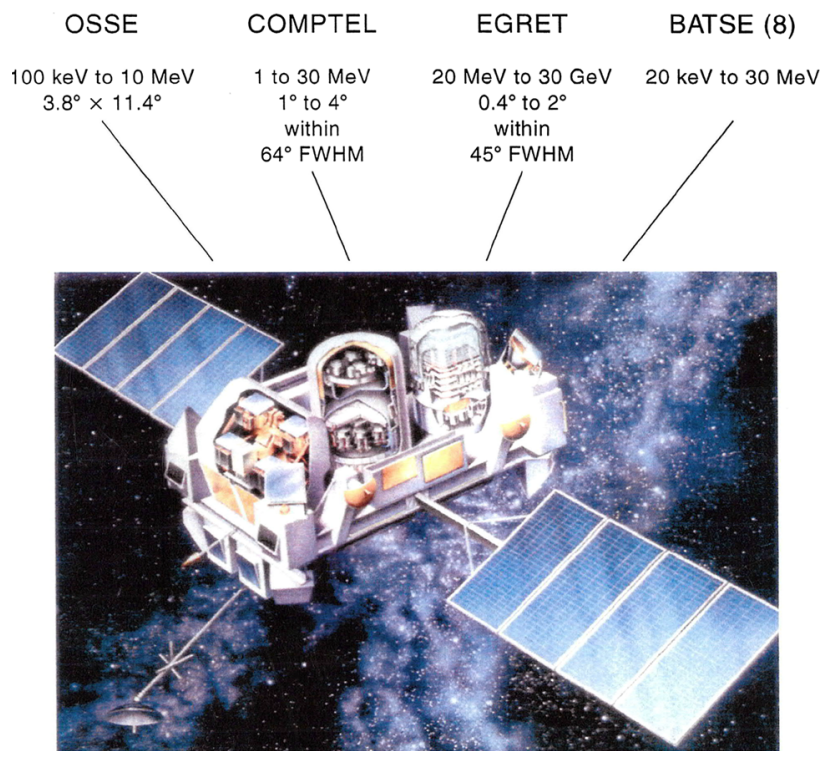

Fig. 18 Schematic view of the Compton gamma-ray observatory with instruments for the $100 \mathrm{keV}$ to $30 \mathrm{GeV}$ range

The Compton Gamma-Ray Observatory belonged to the series of NASA's "Great Observatories" (the first one was the Hubble Space Telescope launched in April 1990). After CGRO, the Chandra X-Ray Observatory was the third (launched in 1999), and the Spitzer Infrared Observatory (launched in 2003) the fourth in this series. Figure 18 shows a schematic view of the Compton Observatory. The platform carries the 4 instruments OSSE, COMPTEL, EGRET, and BATSE. Each of these instruments covers only a limited energy range, but together they cover the energy range from $25 \mathrm{keV}$ to $30 \mathrm{GeV}$.

- OSSE was a collimated scintillation spectrometer for the low-energy range.

- COMPTEL covered the energy range from $700 \mathrm{keV}$ to $30 \mathrm{MeV}$ and consisted of 7 cells of liquid scintillator NE 213 in the upper detector and 14 blocks of NaI crystals in the lower detector. Each cell was viewed by 8 photomultipliers, and each block by 7 photomultipliers. From the relative amplitudes of all photomultipliers viewing one cell or one block, the locations of the interactions could be determined to within about $2 \mathrm{~cm}$ using the Anger Camera principle. Within its wide field-of-view of $64^{\circ}$ (FWHM), COMPTEL had an angular resolution of $1^{\circ}-4^{\circ}$, depending on the energy.

- EGRET was a spark chamber experiment for measuring gamma-rays above $~ 30$ MeV by the pair production process. The pairs or cascades of very high-energy gamma-rays are absorbed in a 20-cm-thick NAI crystal below the spark chamber. Figure 19 shows schematic views of COMPTEL and EGRET. Detailed descriptions of both instruments can be found in $[80,135]$. 

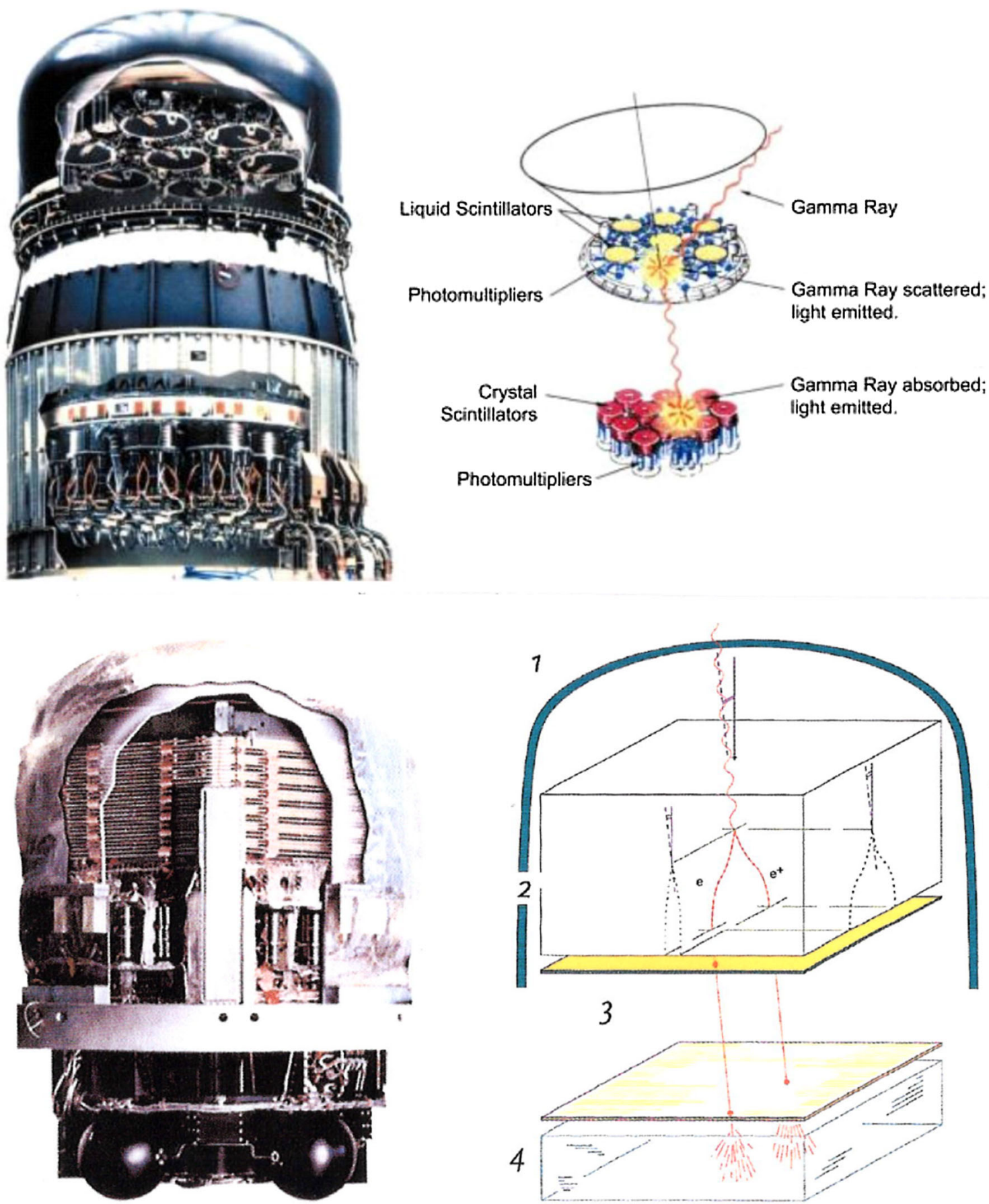

Fig. 19 Instrument schematics and the measurement principles of COMPTEL (top) and EGRET (bottom)

- BATSE consisted of 8 detectors, one at every corner of the platform, and each consisting of a large-area scintillation detector and a smaller, but thicker spectroscopy detector. It was mainly designed to observe and study cosmic gamma-ray bursts.

Figure 20 shows a photograph of the Observatory during its integration at the industrial company TRW. The total weight of the observatory was 17 tons. It was the heaviest astronomical spacecraft which was launched up to that time. When the Space Shuttle Atlantis had reached its orbit, there was a problem with the observatory, because the antenna was stuck and could not be released. Two of the astronauts had to make an unplanned space walk (Fig. 21). One of the astronauts just touched the antenna, and as a result, it got free. This rescued the entire 


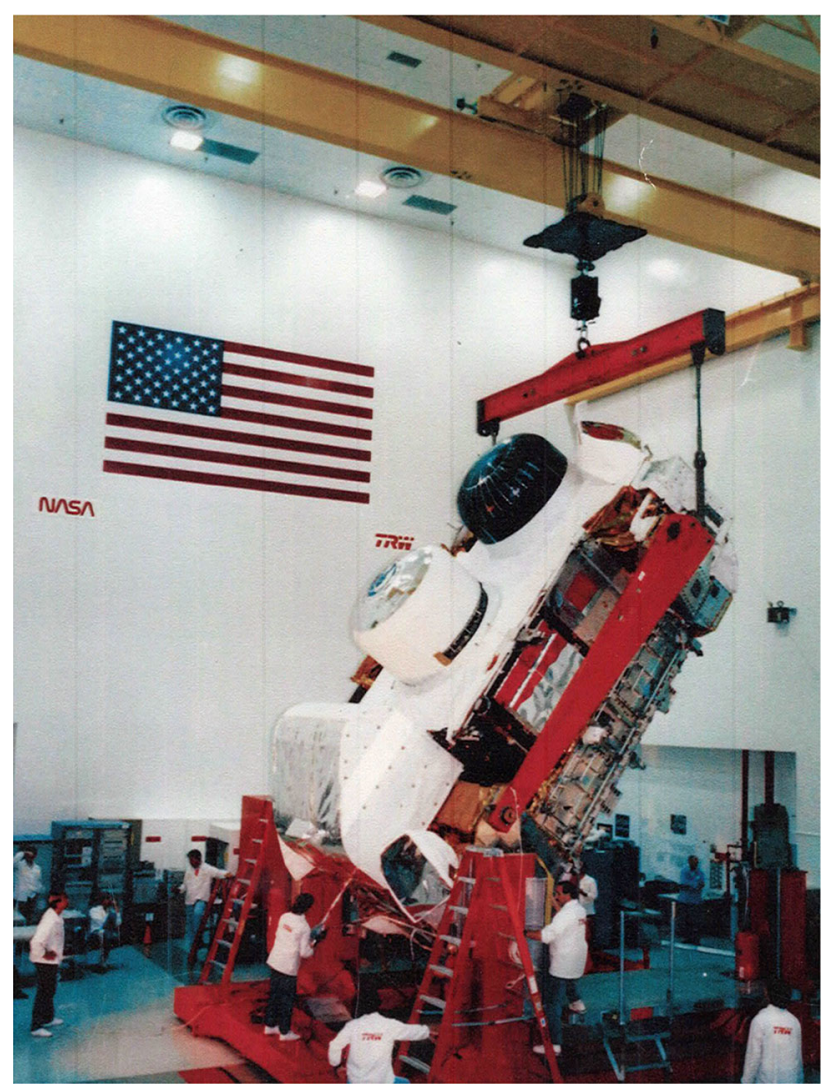

Fig. 20 The CGRO observatory during integration at the industrial company TRW

mission. After being back on Earth the astronauts got a reception by the President of the United States who honoured their achievement.

During the first 18 months of the mission a systematic sky survey was performed (primarily by the fixed-pointing instruments EGRET and COMPTEL). During the following years of the mission the most interesting regions of the sky were studied in more detail (see below).

\subsubsection{EGRET results}

Out of the EGRET sources which could be identified with known sources in other wavebands, 7 were gamma-ray pulsars (isolated neutron stars with strong magnetic fields), about 80 were blazar-type AGN, and one was the Large Magellanic Cloud galaxy. Six of the seven EGRET-detected pulsars were also radio pulsars (the seventh being Geminga). These are exactly those with the largest $\dot{E} / \mathrm{d}^{2}$-values (being an apparent luminosity, where $\dot{E}$ is their rotational energy loss and d the distance). It therefore seemed to be only a question of instrument sensitivity, until more pulsars will be detected at gamma-ray energies. Indeed, this statement has been confirmed by the later Fermi mission. These seven gamma-ray pulsars all have their maximum luminosity at gamma-ray energies. For the six pulsars also seen in the radio band, the gamma-ray luminosity is $4-5$ orders of magnitude higher than the radio luminosity.

Active Galactic Nuclei (AGN) contain supermassive black holes of $10^{6}$ to $10^{9} \mathrm{M}_{\odot}$ in the centre of their galaxies. An accretion disk from surrounding matter is formed around the black hole, and very often one or two relativistic jets are observed perpendicular to the disk. In the canonical interpretation, quasars, blazars, Seyferts, and radio galaxies are different representatives of the class of AGN [159], only the viewing direction of the observer is different. Blazars are those AGN where the observer looks directly into one of the jets. For many of the gamma-ray emitting blazars variability of the order of weeks or months is typical. Even one-day variability has been observed, constraining the emitting region to a very small volume of the size of the solar system. Since EGRET saw gammaray emission only from blazar-type AGN which have jets pointing to the observer, beaming plays the dominant role in the detection. With Lorentz factors of typically $3-20$, the beaming-corrected $\gamma$-ray luminosities are about $10^{46} \mathrm{erg} / \mathrm{s}$ which is about $10^{7}$ times higher than the $\gamma$-ray luminosity of the Milky Way $\left(\sim 10^{39} \mathrm{erg} / \mathrm{s}\right)$.

The detection of gamma-ray emission from the Large Magellanic Cloud has shown that cosmic-rays and interstellar matter interact with each other in this normal galaxy like in our own Milky Way. Gamma-ray emission 


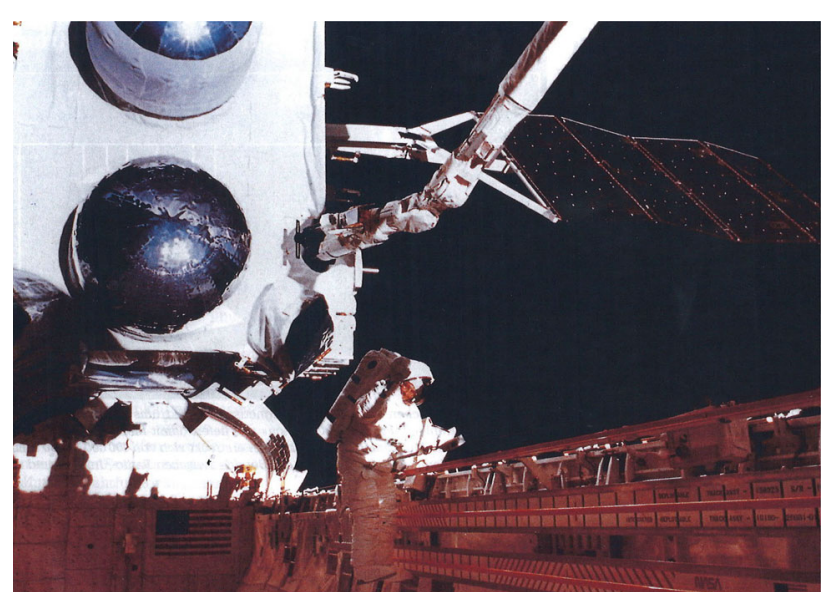

Fig. 21 One of the astronauts (Jay Apt) had to make an unplanned space walk to release the stuck antenna of the Compton Gamma-Ray Observatory

from the Small Magellanic Cloud could not be detected by EGRET. But EGRETs upper limit to its gamma-ray luminosity gave an answer to the long-standing question, whether cosmic rays are galactic or metagalactic. In the early 1970s this was heavily disputed between Burbidge and Ginzburg [21,50]. The answer from EGRETs limit was that cosmic rays are galactic [149]. About 10 years later, Fermi confirmed this conclusion by a real measurement of the SMC-flux [1]. In addition to the sources listed above, EGRET has observed gamma-ray emission from the Sun not only during solar flares (e.g. [83]), but also from the quiet Sun, and from quite a number of cosmic gamma-ray burst sources, which showed surprising properties like GeV-emission still an hour after the onset of the burst [74].

\subsubsection{COMPTEL results}

Out of the 32 COMPTEL gamma-ray sources in the first COMPTEL catalogue [133,140], one was a supernova remnant (SNR) with continuum emission (the Crab nebula), three were galactic radio pulsars (Crab, Vela, and PSR 1509-58), two were galactic accreting black hole systems (Cyg X-1 and Nova Persei 1992), ten were Active Galactic Nuclei, nine were unidentified objects, and six were gamma-ray line sources or possible line sources. In addition, COMPTEL detected and located 31 gamma-ray bursts which happened to occur in the COMPTEL field-of-view. Finally, COMPTEL has also detected gamma-ray emission in continuum and lines from solar flares. Like EGRET, it also observed temporally extended gamma-ray emission from solar flares lasting more than an hour. For instance, the neutron capture line at $2.2 \mathrm{MeV}$ was seen by COMPTEL from several flares over this time interval [126]. Neutrons could be detected and localised by COMPTEL in the same double-scatter mode. The neutron identification is possible by means of the pulse shape discrimination in the upper detector. An all-sky map in the light of high-energy neutrons shows only one single source, the Sun!

COMPTEL has seen three of the seven pulsars detected by EGRET. For the other four EGRET pulsars, the COMPTEL sensitivity was not good enough. Neutron stars are also found in binary systems with a normal star as companion. In these systems, gravitational energy is released by mass accretion from the normal star to the neutron star - in principle similar to the scenario described above for AGN. Such binary systems exist also with white dwarfs and black holes as compact objects instead of the neutron star. Systems with white dwarfs or neutron stars are typical X-ray sources. Systems with a black hole are able to produce gamma radiation up to at least 10 $\mathrm{MeV}$ as observed by COMPTEL from Cyg X-1, the archetype of such a binary. The radiation is supposed to come from the most inner part of the accretion disk around the black hole where the thermal soft X-rays of the disk are Compton-up-scattered by a hot, sometimes relativistic, plasma. The energy spectrum of Cyg X-1 is shown in Fig. 22 from keV energies up to $10 \mathrm{MeV}$ at two different times [113]. The high keV intensity, the so-called high/soft state, represents a state of high accretion rate. The low/hard state is dominated by the Comptonised component. The high-intensity state is relatively rare (about $10 \%$ of the time). There is a direct correlation between the keV and $>\mathrm{MeV}$ flux, and an anticorrelation between the keV- and the $100 \mathrm{keV}$ flux. Different accretion rates obviously trigger different emission geometries (changes of the inner disk radius) and mechanisms (variable Comptonisation, different quasi-periodic oscillations). This changes not only the intensity, but also the overall spectral shape. The other stellar black hole source, Nova Persei 1992 (GRO J0422+32), was detected by COMPTEL only during its strong X-ray outburst, between 1 and $2 \mathrm{MeV}$, near the COMPTEL detection threshold [160]. The resulting spectral shape as measured by OSSE from 50 to $750 \mathrm{keV}$ and by COMPTEL between 1 and $2 \mathrm{MeV}$ is consistent with that of the Cyg X-1 spectrum. 


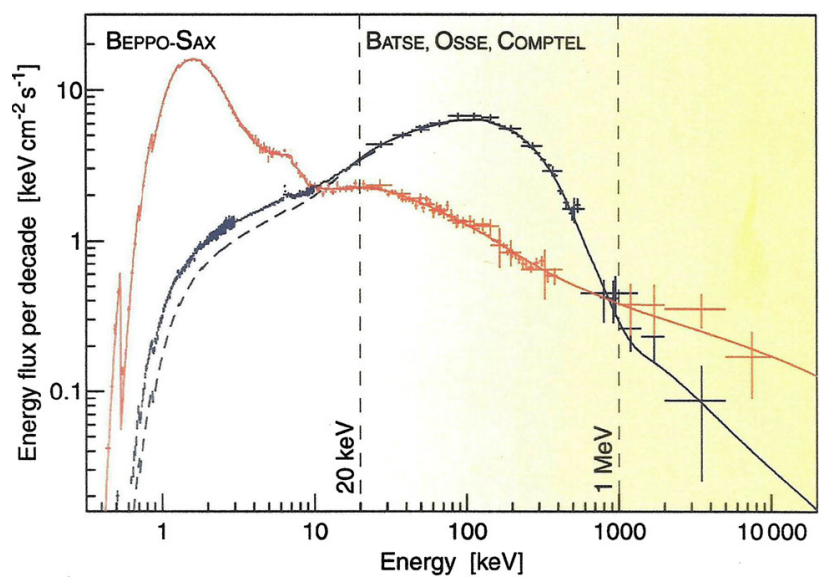

Fig. 22 Energy spectrum of Cyg X-1 in 2 different intensity states: high/soft (red) and low/hard (blue). [Adapted from McConnell et al. [113]]

Table 2 List of radioactive nuclei with gamma-ray line emission

\begin{tabular}{lll}
\hline Decay chain & Mean life (years) & Line energy $(\mathrm{MeV})$ \\
\hline${ }^{56} \mathrm{Ni} \rightarrow{ }^{56} \mathrm{Co} \rightarrow{ }^{56} \mathrm{Fe}$ & 0.31 & $\mathrm{e}^{+}, 0.847,1.238$ \\
${ }^{57} \mathrm{Ni} \rightarrow{ }^{57} \mathrm{Co} \rightarrow{ }^{57} \mathrm{Fe}$ & 1.1 & $0.014,0.122$ \\
${ }^{22} \mathrm{Na} \rightarrow{ }^{22} \mathrm{Ne}$ & 3.8 & $\mathrm{e}^{+}, 1.275$ \\
${ }^{44} \mathrm{Ti} \rightarrow{ }^{44} \mathrm{Sc} \rightarrow{ }^{44} \mathrm{Ca}$ & 78 & $\mathrm{e}^{+}, 0.068,0.078,1.156$ \\
${ }^{60} \mathrm{Fe} \rightarrow{ }^{60} \mathrm{Co} \rightarrow{ }^{60} \mathrm{Ni}$ & $2.2 \times 10^{6}$ & $0.059,1.173,1.332$ \\
${ }^{26} \mathrm{Al} \rightarrow{ }^{26} \mathrm{Mg}$ & $1.1 \times 10^{6}$ & $\mathrm{e}^{+}, 1.809$ \\
\hline
\end{tabular}

COMPTEL has detected ten AGN, nine of which are EGRET blazars [34]. The other one is the radio galaxy Cen A [147], which is probably only seen because it is the nearest of all AGN.

Pioneering observations have been made by COMPTEL in the field of gamma-ray line spectroscopy, which is a powerful tool to explore cosmic element formation processes in novae, supernovae, stellar interiors, and interstellar cosmic-ray interactions (e.g. [38]). Most of the chemical elements are formed by nuclear interactions in the interior of stars or during supernova (SN) explosions. During these processes not only stable, but also radioactive isotopes are produced. Some of them are gamma-ray emitters, and these can be used to study the cosmic element production processes. Table 2 lists the most important isotopes from nucleosynthetic decay chains, which lead to gamma-ray emission. The decay times range from a few months to more than a million years. The short-lived isotopes allow us to study individual nucleosynthesis sites, and the long-lived ones provide global information about nucleosynthesis processes integrated over a long time period. The ${ }^{56} \mathrm{Co}$ lines from the type II supernova 1987A in the Large Magellanic Cloud had already been detected prior to the launch of the Compton Observatory [101], and OSSE finally succeeded in detecting the ${ }^{57}$ Co lines from this SN as well [100].

The highlight in the field of long-lived radioactive isotopes is COMPTEL's all-sky map in the light of the 1.809 MeV line from radioactive ${ }^{26} \mathrm{Al}$ (Fig. 23, [39,118]). Already discovered by the HEAO-C satellite in 1982 [105] as the first cosmic radioactivity, and given its half life-time of about $10^{6}$ years (short in comparison with the age of our galaxy), it clearly demonstrated that nucleosynthesis is still actively taken place in the Galaxy. Detailed imaging spectroscopy thus offers an unprecedented opportunity to identify these nucleosynthesis sites. COMPTEL's first-ever all-sky map in the light of a radioactivity gamma-ray line provided information on the spatial distribution of ${ }^{26} \mathrm{Al}$ production in the Milky Way during the last million years. From Fig. 23 we learned that the galactic plane is a strong source of $1.809 \mathrm{MeV}$ line emission, especially around the Galactic Center and in the Cygnus, Carina, Vela and Orion regions. All these regions contain young star-forming regions with high supernova activity. This correlation initiated first detailed studies on the basis of stellar-group population synthesis evolution models, among others in combination with 1-dimensional models of expanding superbubbles around the OB associations [124], or the isotope production in star-forming regions to obtain the light curves of $\gamma$-ray line emission due to radioactive decay of ${ }^{26} \mathrm{Al},{ }^{60} \mathrm{Fe}$ and the $\mathrm{e}^{-} \mathrm{e}^{+}$annihilation line [31]. These findings, combined with multi-frequency model fitting, support the earlier hypothesis that massive stars and their descendent supernovae are the dominant sources of interstellar ${ }^{26} \mathrm{Al}$ as observed by COMPTEL (see more details about its origin from high spectral resolution measurements with the INTEGRAL mission in Sect. 3.3.1). An extensive review of this 
Fig. 23 COMPTEL all-sky map in the light of radioactive ${ }^{26} \mathrm{Al}[123]$

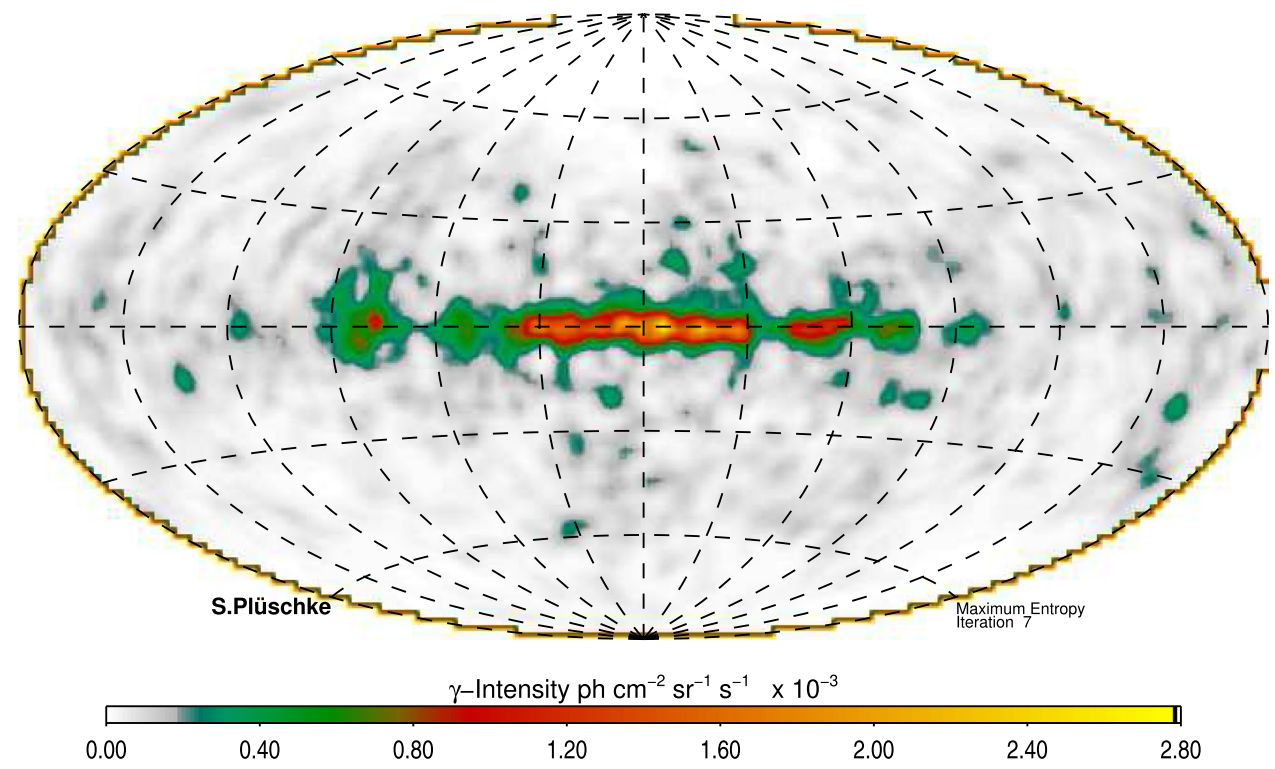

"new astronomy", including the history of the ${ }^{26} \mathrm{Al}$ line emission observations and the status of the theoretical understanding is given in Prantzos \& Diehl [125].

A radioactive gamma-ray line which was discovered by COMPTEL for the first time, is the $1.157 \mathrm{MeV}$ line from radioactive ${ }^{44} \mathrm{Ti}$. This isotope has a relatively short decay time of 90 years and is supposed to be produced only in supernovae. This line, therefore, has the potential to discover hidden, so far unknown supernova-remnants, which are not older than a few hundred years. COMPTEL first detected the $1.157 \mathrm{MeV}$ line from Cas A, the youngest (330 years old) known remnant in our Galaxy [76]. From the measured line flux a mass of $1-2 \times 10^{-4}$ solar masses of ${ }^{44} \mathrm{Ti}$ was derived to have been produced at the time of the explosion. This value is roughly consistent with expectations. Later, COMPTEL found the signature of ${ }^{44} \mathrm{Ti}$ line emission from a second sky region which was located near the Vela supernova remnant [77]. Since ${ }^{44} \mathrm{Ti}$ is expected to be produced only in supernova explosions, this source should be a supernova-remnant as well, but due to the short ${ }^{44} \mathrm{Ti}$ decay time, the source could not be the well-known, 12.000-year-old Vela SNR. Indeed, independently of the gamma-ray observations, Aschenbach [7], with ROSAT X-ray data, discovered a $2^{\circ}$ diameter SNR at the position of the ${ }^{44} \mathrm{Ti}$ line, in front of the southeast corner of the $8^{\circ}$ Vela SNR. This remnant, called Vela-Junior (RX 0852.0-4622), showed a particularly high temperature of $>3 \times 10^{7} \mathrm{~K}$, suggesting a young age of only $\sim 1000 \mathrm{yrs}$, consistent with the detection of ${ }^{44} \mathrm{Ti}$. Since this discovery, much more information on the remnant has been obtained from X-ray and radio observations with the result that the ${ }^{44} \mathrm{Ti}$ signal is presently considered unlikely, after its firm COMPTEL detection had already been withdrawn $[133,140]$. Curiously, even the accurate distance to Vela-Junior and its age are still heavily debated (e.g. [3]).

Only one week after the launch of CGRO, the type Ia supernova SN 1991T was discovered, one of the brightest and closest supernovae of that decade. COMPTEL observed the sky region of SN 1991Ts for two weeks each at 2 and 5.5 months, respectively. No emission from the ${ }^{56} \mathrm{Co}$ decay at $847 \mathrm{keV}$ and $1.238 \mathrm{MeV}$ was found, with $2 \sigma$ upper limits of $3 \times 10^{-5} \mathrm{ph} / \mathrm{cm}^{2} / \mathrm{s}$ [103,132]. These upper limits allowed to exclude two out of the more than a dozen proposed SN models.

\subsubsection{Synergy between CGRO instruments}

A less appreciated feature of the CGRO was the coordinated scientific analysis of data from different instruments, despite the fact that by construction they observed the same source(s) from the same place and the same time. One of the immediate similar findings is the fact that the EGRET and COMPTEL all-sky maps, created from the sum of the data of the first 18 months, look similar, despite their very different energy ranges (>100 MeV vs. 1-30 $\mathrm{MeV}$, respectively). Figure 24 displays the continuum all-sky maps of EGRET [48,79] and COMPTEL [132]. In both maps, the Milky Way is clearly seen against the rest of the sky, and several prominent sources are labelled in Fig. 24. The region around the Galactic centre is especially bright, with the central point-like high-energy emission speculated to be due to individual sources, presumably pulsars [111]. Most of the observed galactic emission is diffuse in nature and produced in interstellar space. In addition, a number of objects were also seen at mid and high latitudes. These were supposed to be predominantly of extragalactic origin. Many of those have been identified as a subclass of quasars - so-called blazars: their discovery by EGRET was one of the great surprises of the CGRO mission.

However, there are also marked differences between the two sky maps: some sources are only visible at high energies like the Geminga [110] and Vela pulsar [84], others only at low energies (e.g. Cas-A, Cyg X-1). Figure 25 
Fig. 24 All-sky continuum maps of COMPTEL (top) and EGRET (bottom)

\section{COMPTEL 1-30 MeV}

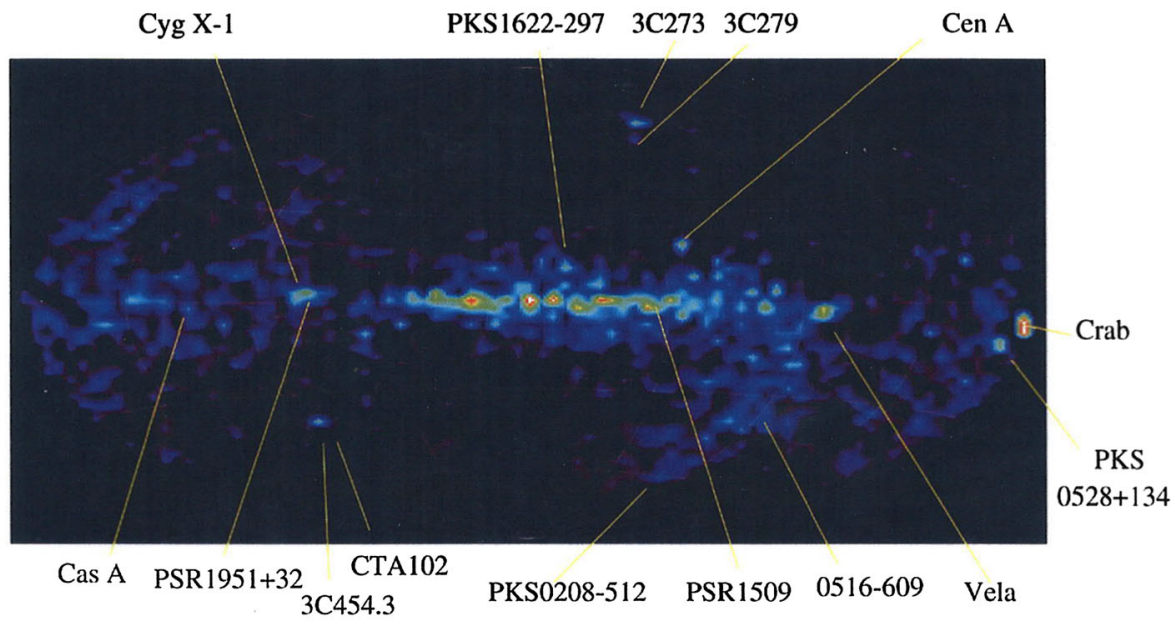

EGRET > $100 \mathrm{MeV}$

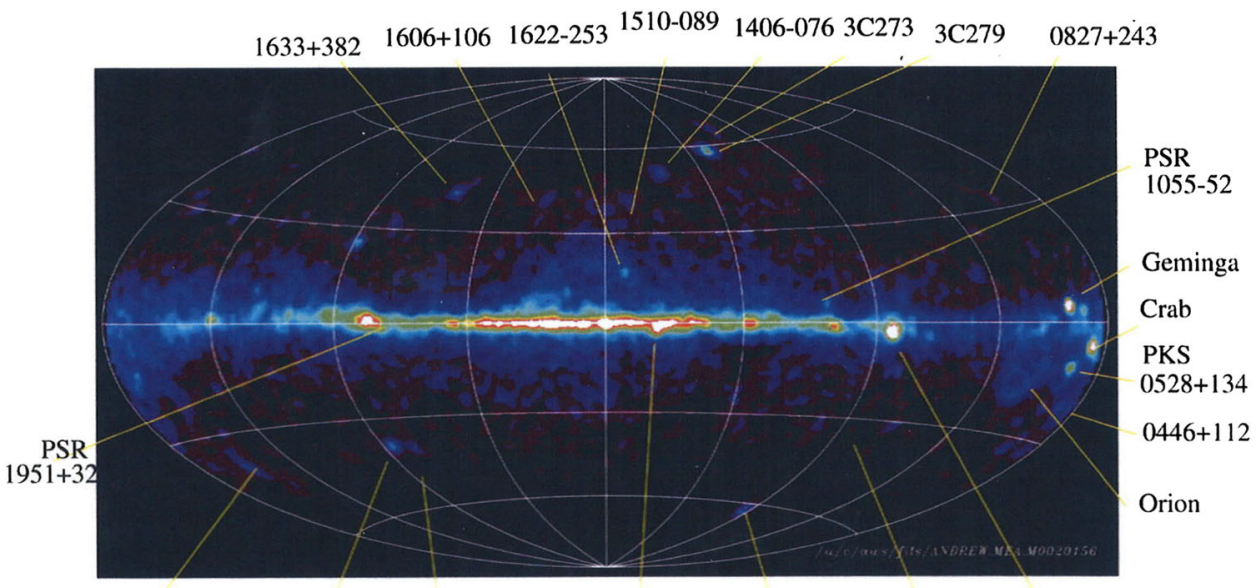

$\begin{array}{llllll}0235+164 & 3 \mathrm{C} 454.3 & 2230+114 & \text { PSR 1706-44 PKS 0208-512 LMC } & \text { Vela }\end{array}$

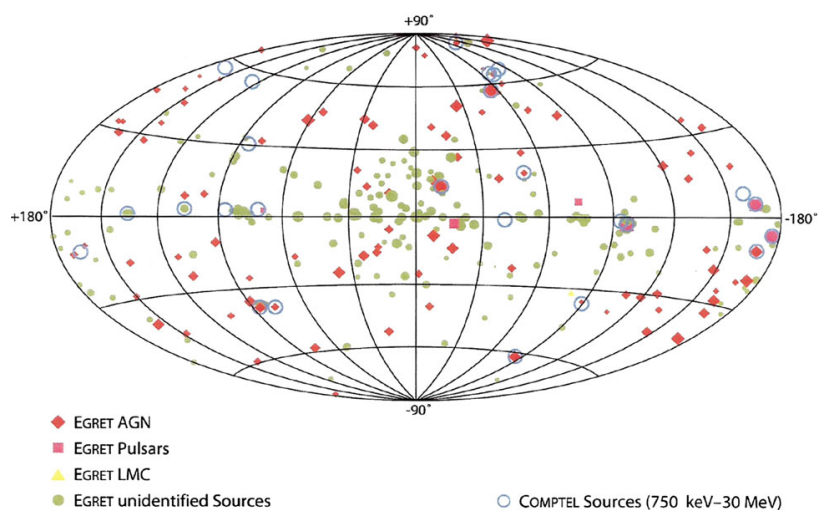

Fig. 25 All-sky maps of the COMPTEL and EGRET sources 


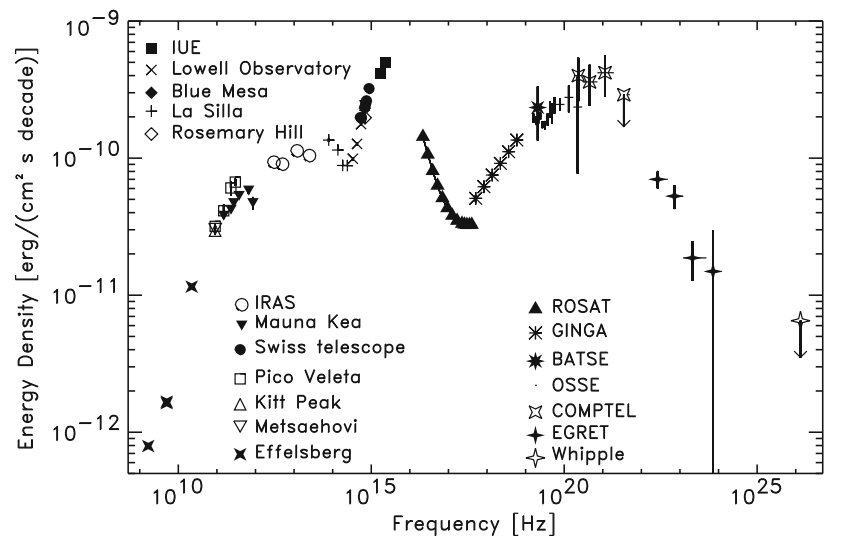

Fig. 26 Broad-band spectral energy distribution of the quasar 3C 273, build from quasi-simultaneous observations. [Adapted from Lichti et al. [104]]

is an all-sky map of the COMPTEL and EGRET sources. This map is derived from Fig. 24 after subtracting the diffuse galactic and cosmic background radiation [68,133,140]. In total, about 300 gamma-ray emitting objects were detected by both instruments together. Out of these, nearly 100 could be identified with known celestial objects. The remaining two-thirds remained unidentified until Fermi/LAT observations (see below).

The widely known multi-wavelength analysis of several CGRO instruments concerns blazars. When combining the blazar spectra simultaneously measured by OSSE, COMPTEL, and EGRET, a spectral turnover becomes visible at $\mathrm{MeV}$ energies for a number of sources. A nice example is 3C 273 (Fig. 26, [104]). In addition, COMPTEL found indications for an unexpected class of so-called $\mathrm{MeV}$ blazars among the nine AGN, which occasionally show bumps in the 1-10 MeV range above a simple extrapolation of the simultaneously measured EGRET-spectra to lower gamma-ray energies $[18,19]$. Finally, it should be mentioned that no Seyfert galaxy has been observed at gamma-ray energies, though there had been reports in the 1970s from balloon flights about possible detections at $\mathrm{MeV}$ energies. OSSE has searched for $\mathrm{MeV}$ gamma-ray emission of about 25 Seyferts, but without success. All Seyfert spectra cut-off at about $300 \mathrm{keV}$, consistent with upper limits, determined by COMPTEL at MeV energies.

In addition to the point sources listed above, COMPTEL has also analysed in detail diffuse continuum emission from interstellar space in the Milky Way as well as the diffuse cosmic background radiation. The latter has already been discussed in detail in chapter 3.1.3 (see Fig. 13). The main conclusion from these studies was that the famous $\mathrm{MeV}$ bump originally discovered by Apollo XV was an artefact caused by background radiation. A combined spectrum of the real cosmic background spectrum from $1 \mathrm{keV}$ to $100 \mathrm{GeV}$ including data from ASCA, HEAO-A2, HEAO-A4, COMPTEL, SAS-2, and EGRET was presented in Sreekumar et al. [145]. In their Fig. 6 (reproduced here in Fig. 27) an attempt is made to explain the total measured spectrum as superposition of contributions from unresolved Seyferts, SN type Ia, and blazars.

From the combined analysis of OSSE, COMPTEL, and EGRET data on the interstellar gamma-ray emission between $50 \mathrm{keV}$ and $10 \mathrm{GeV}$ some new questions arose. When in the 1970s only measurements from SAS-2 and COS-B were available around $100 \mathrm{MeV}$, the origin of the interstellar continuum gamma-ray emission seemed to be explained as a mixture of $\pi^{\circ}$-decay from cosmic-ray nuclei interactions, bremsstrahlung, and inverse Compton emission of cosmic-ray electrons. With CGRO, additional measurements at lower and higher energies showed that the origin of the diffuse galactic emission was far from understood [152]. Around $100 \mathrm{MeV}$, the measurements were still in agreement with the earlier theoretical ideas, but at high energies above $1 \mathrm{GeV}$ the measured intensity was higher than expected. It had been suggested that the energy spectra of cosmic-ray nuclei and electrons - as measured near Earth - are not representative for the entire galaxy, but that their spectra are generally harder (more protons at higher energies) than locally measured. In this case the $\mathrm{GeV}$-excess could be explained. Also, at lower energies - below about $30 \mathrm{MeV}$ - the measured intensity was higher than expected. This part of the spectrum was supposed to be explained by cosmic-ray electron interactions. Here, a higher electron intensity would be needed, which, however, would be in conflict with the measured radio synchrotron intensity. A suggested alternative was that the measured intensity below $30 \mathrm{MeV}$ is not only of purely diffuse origin, but that a component of unresolvedso far unknown - point sources has to be added. Later Fermi observations indeed show that the diffuse gamma-ray background in the $100 \mathrm{MeV}$ to $1 \mathrm{TeV}$ can be explained by emission from unresolved misaligned AGN, blazars, and star-forming galaxies [36]. 
Fig. 27 Energy spectrum of the diffuse cosmic background radiation containing the COMPTEL and EGRET

measurements, modelled as the sum of Seyfert 1 (dot-dashed), Seyfert 2 (dashed), steep-spectrum quasars

(dot-dot-dot-dashed), Type Ia supernovae (dot), and blazars (dashed) which are modelled with a broken power-law with photon indices of -1.7 and -2.1 , breaking at $\sim 4 \mathrm{MeV}$. [From Sreekumar et al. [145]]
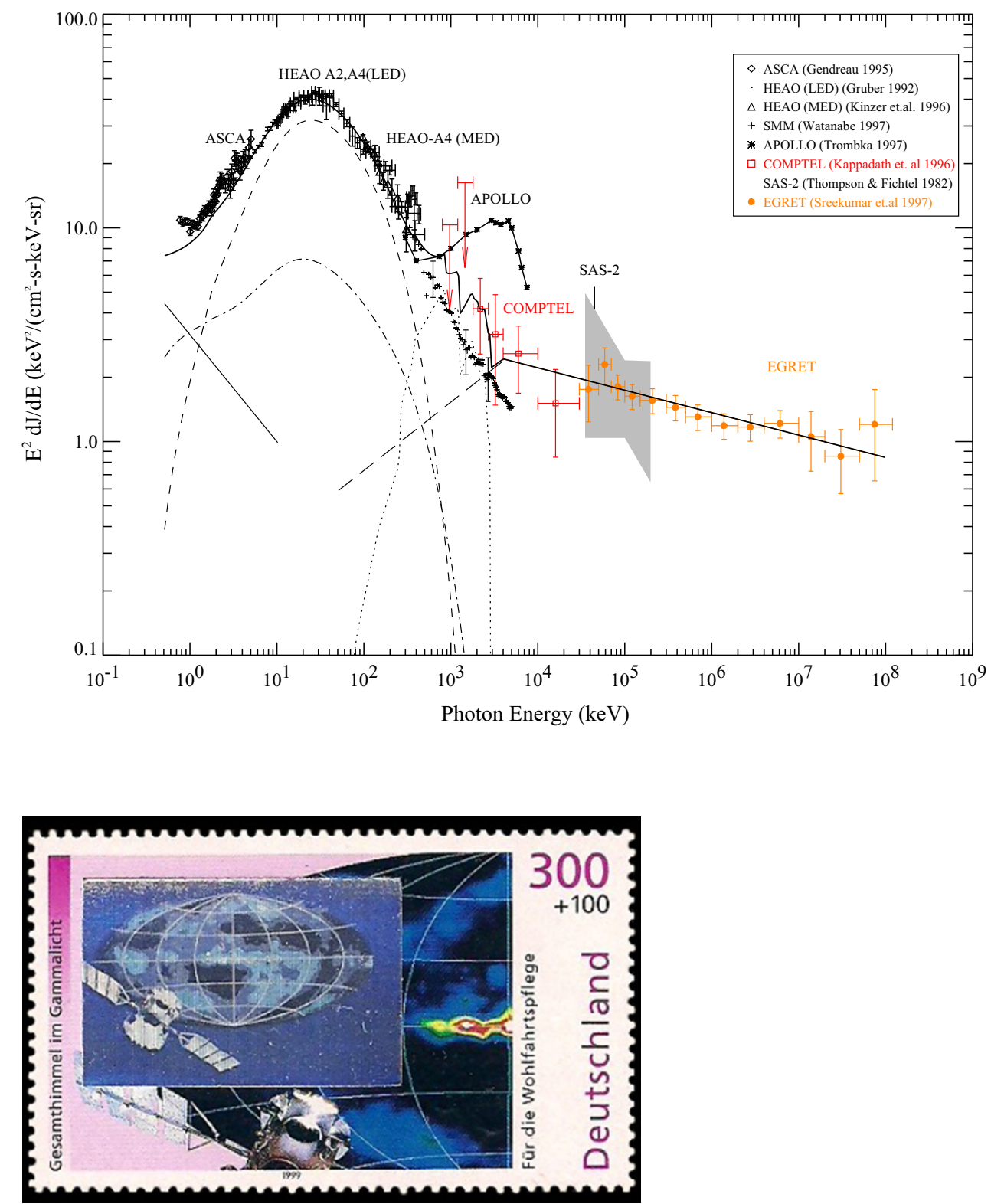

Fig. 28 German stamp from 1999 (value of 300 Pfennig = 3.00 DM) showing the COMPTEL all-sky map. Its validity as stamp ceased in 2002 after the introduction of the Euro as common European currency

\subsubsection{Public attention to the Compton mission in Germany}

At the end of this chapter on results of the Compton mission a few words shall be added about the public attention which EGRET and especially COMPTEL received in Germany. First, there were quite a number of articles in popular scientific Journals. Especially the question of the origin of the chemical elements - one of the main topics of COMPTEL - was of special interest, also in the light of religious aspects. Second, the instrument size and complexity of COMPTEL created technical interest, and consequently the full-size structure model of COMPTEL with one original D-1 and D-2 detector module was prepared for exhibition in the Deutsches Museum in Munich. Third, a stamp with the COMPTEL sky map was issued by the German post office (Fig. 28). Also, for the entire field of gamma-ray astronomy, our work at the Max-Planck Institute was very fruitful: During Schönfelder's professional life, a total of $27 \mathrm{PhD}$ - or Master students got their degree from work with COMPTEL or EGRET. A few of them finally got a professorship position in Germany, or abroad. They carried on our experience from the Compton Observatory into future missions. According to NASA/ADS, about 1200/1100/350/250 refereed papers were published on BATSE/EGRET/COMPTEL/OSSE-results, respectively, and the average over all refereed CGRO publications is 40 citations/paper, quite amazing even for present-day standards. 


\subsection{Gamma-ray observations with INTEGRAL and Fermi}

Already during the operational phase of GRO we started in the mid-1990s to prepare for future missions. This concerned two missions actively discussed, namely GLAST/Fermi in the USA, and INTEGRAL in Europe. In addition, we also started a conceptually new design, combining the Compton detection mode with the low-energy pair creation mode, leading to the MEGA prototype.

\subsubsection{INTEGRAL}

In 1993, INTEGRAL (International Gamma-Ray Astrophysics Laboratory) was selected by ESA as a medium-size mission within its Horizon 2000 program. INTEGRAL carries 4 different instruments: the 2 main instruments are the Spectrometer on INTEGRAL (SPI) and the Imager on-board the INTEGRAL Satellite (IBIS), which are complemented by 2 small monitors, the Joint European X-Ray Monitor JEM-X, and the Optical Monitoring Camera OMC.

SPI (20 keV to $8 \mathrm{MeV}$ ) is devoted to high-resolution spectroscopy with modest imaging capability. Within its fully coded field-of-view of $16^{\circ}$, its angular resolution is about $2.5 \mathrm{FWHM}$ [161]. SPI is able to measure the profiles of gamma-ray lines to an accuracy of about $2-3 \mathrm{keV}$. The knowledge of the line profile allows a detailed study of the physical conditions of the sky regions in which the lines are produced, like temperature, expansion velocity, ionisation degree, and emission geometries. A similar experiment - called GRSE - was originally planned for the Compton Observatory, but was never realised.

The imager IBIS (15 keV to $10 \mathrm{MeV}$ ) has an excellent angular resolution of 12 arcmin within its fully coded field-of-view of $8^{\circ}$ [158]. The 2 monitor instruments JEM-X and OMC allow parallel monitoring of the gamma-ray sources in the adjacent $\mathrm{X}$-ray $(3 \mathrm{keV}$ to $35 \mathrm{keV})$ band and at optical wavelengths.

SPI, IBIS, and JEM-X are all three based on the coded aperture imaging technique. SPI uses Germanium detectors, IBIS CdTe and CsI-detectors, and JEM-X micro-strip Xenon gas detectors. OMC consists of a passively cooled CCD in the focal plane of a 50-mm lens.

Because of the interesting results obtained with COMPTEL in the field of gamma-ray line spectroscopy, MPE decided to participate in INTEGRAL-SPI. The two main institutes responsible for SPI are the Centre d'Etude Spatiale des Rayonnements in Toulouse, France, and MPE in Garching, Germany, with the two initial Co-Principal Investigators G. Vedrenne and V. Schönfelder. Other scientific groups contributing to SPI are from Saclay, France, and from Spain, Belgium, Italy, UK, and the USA.

Figure 29 shows a schematic view of SPI. The core of the telescope is the Germanium detector at the bottom of the telescope which consists of 19 individual crystals. The entire detector is contained in a cryostat which is cooled down to 80-85 K. The bottom of the Ge-detector and the sides are surrounded by a massive BGO-anticoincidence shield (ACS) which defines the field-of-view by shielding against background radiation. At the top of the telescope $-171 \mathrm{~cm}$ above the Ge-detector plane - is the passive coded aperture mask made of tungsten.

Figure 30 shows the final schematic view of INTEGRAL with all its instruments. This final concept differs somewhat from that proposed to ESA in 1993. Most of the changes were made after a major involvement of the USA could not be realised, and the UK had decided not to participate in the building of the scientific payload. The INTEGRAL launch took place in October 2002 from Baikonur in Kasachstan with a Russian Proton rocket, putting INTEGRAL into a highly excentric 72-h orbit. A photograph taken during the mounting of INTEGRAL onto the rocket is shown in Fig. 31.

With MPE's strong gamma-ray line astrophysics tradition from COMPTEL, this remained the focus for SPI studies. This was further justified by the major new measurement capability of SPI, namely its spectral resolution of 100-1000 (e.g. $3.1 \mathrm{keV} \mathrm{FWHM} \mathrm{at} 1809 \mathrm{keV}$ ), opening valuable opportunities to determining absolute and direct measurements of fluxes, Doppler shifts and broadenings, as well as kinematically or relativistically distorted line shapes from a large variety of sources.

Astrophysical gamma-ray measurements up to $\sim 50-100 \mathrm{MeV}$ are strongly affected by background, both by external radiation as well as secondary, induced background in the satellite and instrument. Given the high spectral resolution of SPI, one of the first important tasks was to attempt to understand the measured background spectrum including gamma-ray lines. A first spectral and temporal characterisation of the data revealed more than 300 emission lines, and provided identifications for nearly 220 of these [171]. Over the years, a spectral parameter database was developed, based on the long-term monitoring of the SPI germanium detectors [43]. This was finally used to build a self-consistent and broadly applicable background model for typical science cases of INTEGRAL/SPI [142] which accounts for the many differently variable background components. An attempt to relate some of these major components to source types is shown in Fig. 32.

An immediate continuation of MPE's gamma-ray line tradition was the study of the ${ }^{26} \mathrm{Al} 1.8 \mathrm{MeV}$ line. This line originates from the radioactive decay to $\mathrm{Mg}$, predominantly in massive stars, and its decay half-life time of 700.000 yrs is well suited to map the star formation and wind activity of massive stars over the last few million years. In particular, the half-life time is (i) long enough for ${ }^{26} \mathrm{Al}$ to escape from the star where it is produced in 


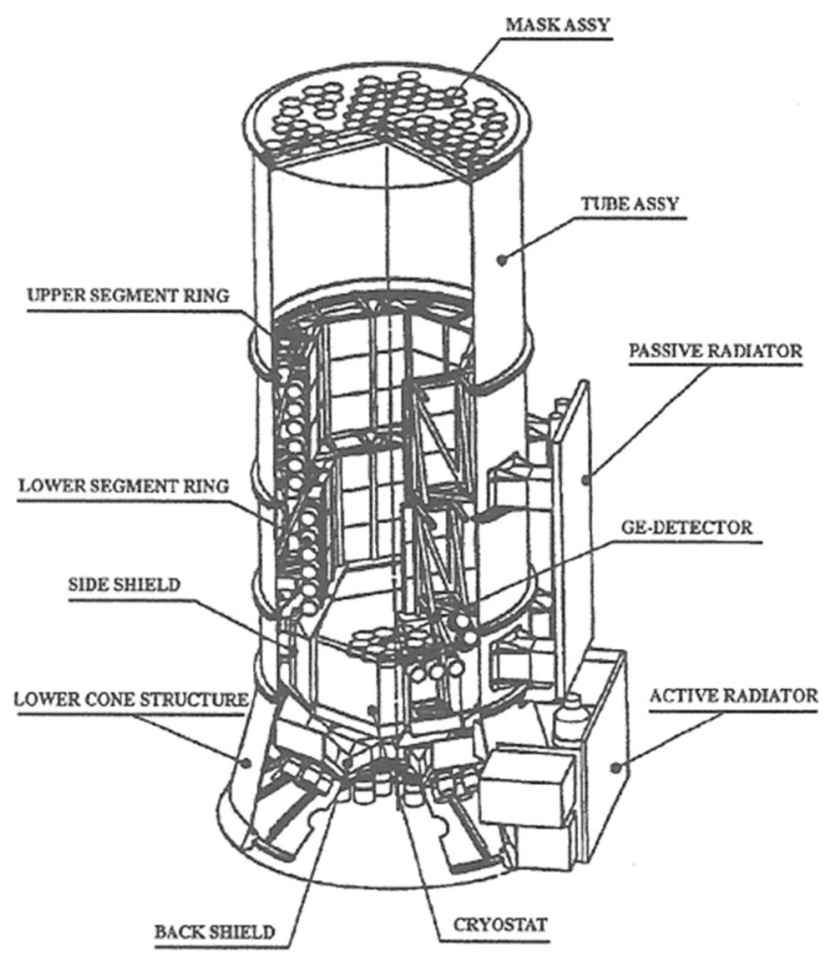

Fig. 29 Schematics of the INTEGRAL spectrometer SPI

Fig. 30 Schematics of the INTEGRAL satellite with its instruments

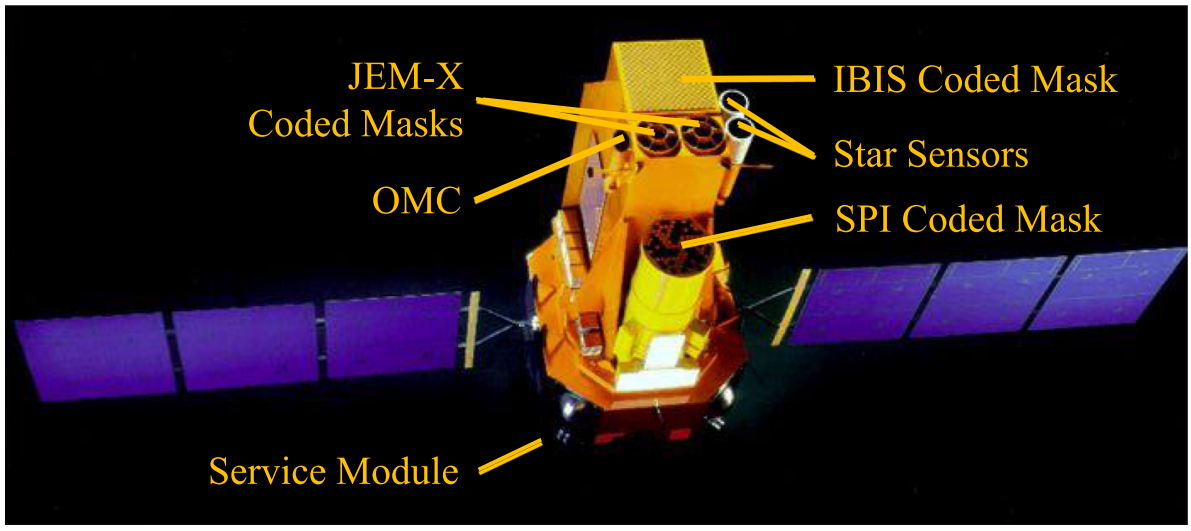

both stellar winds and supernovae, and (ii) short enough to not be completely homogenised in the Galaxy before it decays. This makes it an ideal tracer of the dynamics of ongoing nucleosynthesis in the Galaxy. Indeed, the high spectral resolution of SPI now allowed to measure the 'galactic rotation' of the ${ }^{26} \mathrm{Al}$ emission, demonstrating its large-scale, Galaxy-wide distribution [40]. Surprising, an excess velocity of more than $100 \mathrm{~km} / \mathrm{s}$ is measured (Fig. 33) which can be explained by a global wind blow-out into the forward direction away from spiral arms into the inter-arm region [96]. Simulations show that the density at the nucleosynthesis site is possibly lower than that of the typical interstellar medium: in that case the high wind expansion velocities of $\sim 1000 \mathrm{~km} / \mathrm{s}$ prevail for a longer time before the radioactive material is decelerated by the interstellar gas [93]. This has interesting implications for angular-momentum transfer in the disk-halo system and consequently also radial gas flows.

In February 2014, a type Ia supernova (called SN 2014J) exploded in a nearby galaxy, allowing unprecedented $\gamma$-ray measurements. The ${ }^{56} \mathrm{Ni}$ freshly produced in the supernova is understood to power the optical light curve, because it emits $\gamma$-rays upon its radioactive decay first to ${ }^{56} \mathrm{Co}$ and then to ${ }^{56} \mathrm{Fe}$. Already a few days after the explosion the characteristic $158 \mathrm{keV}$ and $812 \mathrm{keV}$ lines of the ${ }^{56} \mathrm{Ni}$ decay appeared [41]. This was much earlier than model predictions of at least 3 months, since ${ }^{56} \mathrm{Ni}$ is commonly believed to be buried deeply in the expanding supernova cloud. This early appearance suggests both an asymmetric explosion event and the production of ${ }^{56} \mathrm{Ni}$ farther out in the ejecta than predicted. A plausible explanation is that a belt of helium from the companion star is accreted by the white dwarf, where this material explodes and then triggers the supernova event [41]. The 847 keV and $1238 \mathrm{keV}$ lines from the ${ }^{56}$ Co decay were detected after $2-3$ months with significant Doppler broadening [42], 


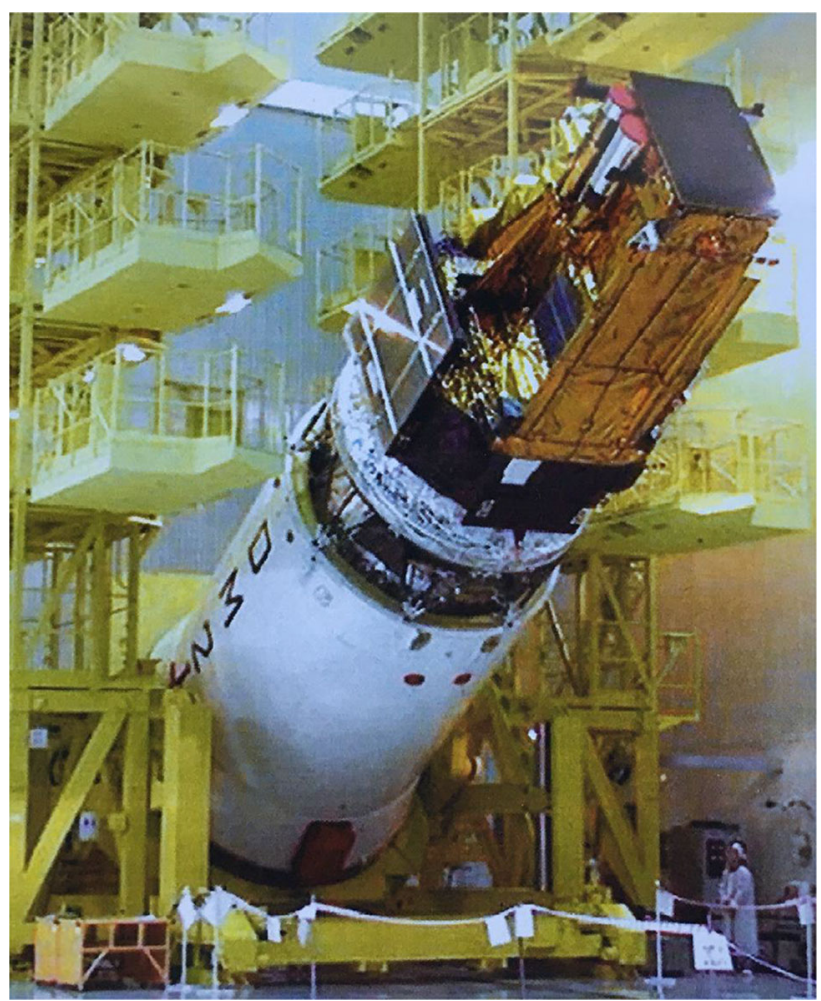

Fig. 31 During the assembly of INTEGRAL onto the Proton rocket in Baikonur (Kasachstan)

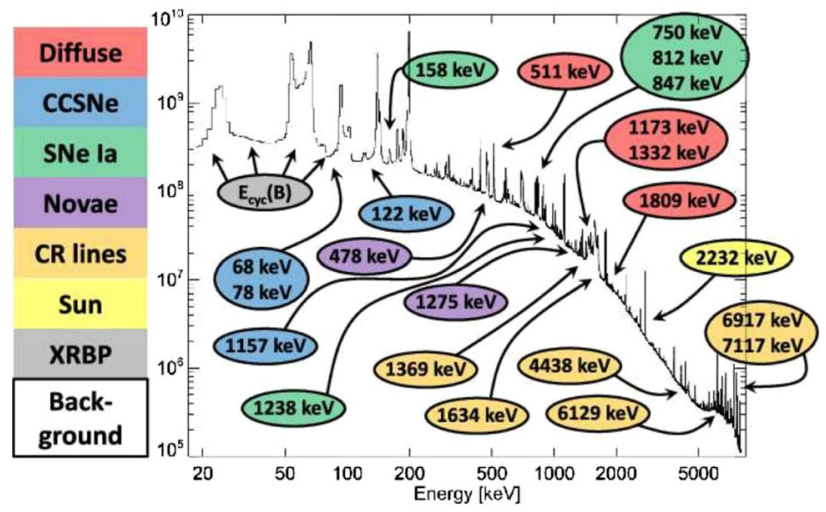

Fig. 32 INTEGRAL/SPI background spectrum (black histogram) from 16 mission years. In colours, prominent $\gamma$-ray lines from a variety of astrophysical sites are indicated. Note that there is substantial overlap between $\gamma$-ray lines from different source types. [From Siegert et al. [142]]

consistent with model expectations for the kinematics of the ${ }^{56} \mathrm{Ni}$ ejecta. The temporal evolution of the emission spectrum turned out to be complex and irregular until the supernova became fully transparent to $\gamma$-rays, another surprise which this first-of-its-kind INTEGRAL observation revealed [42]. This direction-dependent $\gamma$-ray emission implies that our model concepts are not yet physically consistent, and affected by additional, partly variable components. This could have serious implications for cosmology, where SN Ia are used as standard candles.

Another area of active research was the origin of the $511 \mathrm{keV}$ electron-positron annihilation emission. This was mapped already with OSSE on CGRO, but its spatial distribution, even in 2-dimensions on the sky, remained under debate. Improved mapping with SPI constrained the extent of the emission in the Galactic bulge [173], leading to the discovery of a distinct asymmetry in the $511 \mathrm{keV}$ line emission (Fig. 34) coming from the inner Galactic disk ( 10-50 ${ }^{\circ}$ from the Galactic Centre) [174]. This asymmetry resembles an asymmetry in the distribution of low mass X-ray binaries with strong emission at photon energies $>20 \mathrm{keV}$ ('hard' LMXBs), indicating that they may be the dominant origin of the positrons. This implied that up to a few times $10^{41}$ positrons/second escape from a typical hard LMXB. This was spectacularly proven with INTEGRAL observations of a strong flaring activity of 


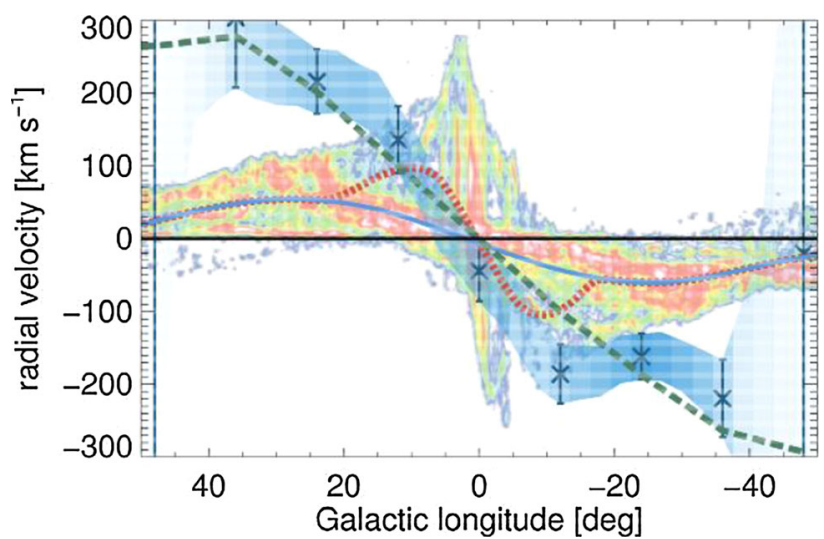

Fig. 33 Longitude-velocity diagram comparing ${ }^{26} \mathrm{Al}$ velocities (crosses) with other object distributions in our Galaxy, i.e. molecular CO gas in the ridge (colour scale overlay), free electron density (blue), a 1:3 ratio between bar and disk (red), and a new leading-edge blow-out of $\sim 200 \mathrm{~km} \mathrm{~s}^{-1}$. [From Kretschmer et al. [96]]
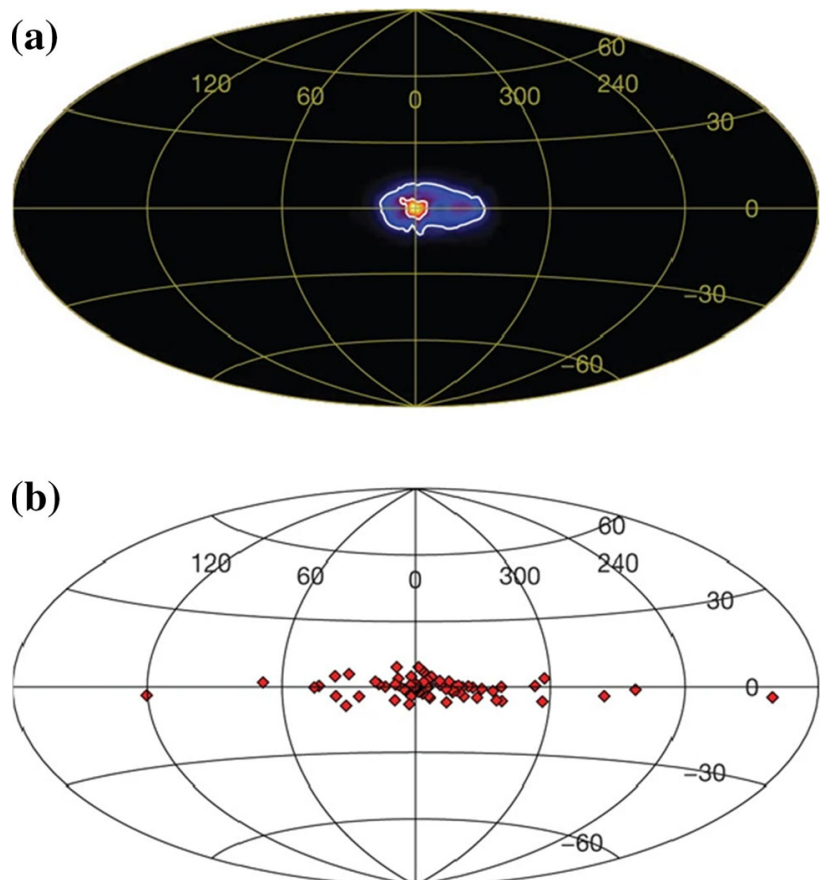

Fig. 34 The $511 \mathrm{keV}$ line map as observed with INTEGRAL/SPI (top) and the sky distribution of hard LMXBs detected at energies above $20 \mathrm{keV}$ with INTEGRAL/IBIS, showing the resemblance to that of the $511 \mathrm{keV}$ annihilation line. [From Weidenspointner et al. [174]]

the microquasar V404 Cygni in 2015. The observed temporary strong $511 \mathrm{keV}$ flux (Fig. 35) corresponded to a positron creation rate of $10^{42} \mathrm{~s}^{-1}$, a few percent of the total flaring luminosity [141]. About 10 active microquasars at any time would already reproduce the steady-state annihilation rate in the inner Galaxy. With duty cycles of the order of $10^{-3}$ as observed for V404 Cygni, $\sim 10^{3}-10^{4}$ accreting black hole binaries of this type would be required, consistent with population synthesis estimates. This makes microquasars a viable source of the electron-positron plasma responsible for the bright diffuse emission of annihilation $\gamma$-rays in the bulge region of our Galaxy [141], thus eliminating the need for more exotic explanations, such as those involving dark matter.

Already earlier, Martin et al. [106] had concluded that positrons from nucleosynthesis reactions alone cannot account for the observed annihilation emission, thus requiring an additional component to explain the strong bulge contribution. Using the GALPROP propagation code [150] to simulate the transport and annihilation of radioactivity positrons in our Galaxy, several possible propagation scenarios were explored to cover the large uncertainties on the transport of $\sim \mathrm{MeV}$ positrons in the interstellar medium. Comparing the predicted intensity distributions to the INTEGRAL/SPI observations, Martin et al. [106] estimated that at least half of the positrons 


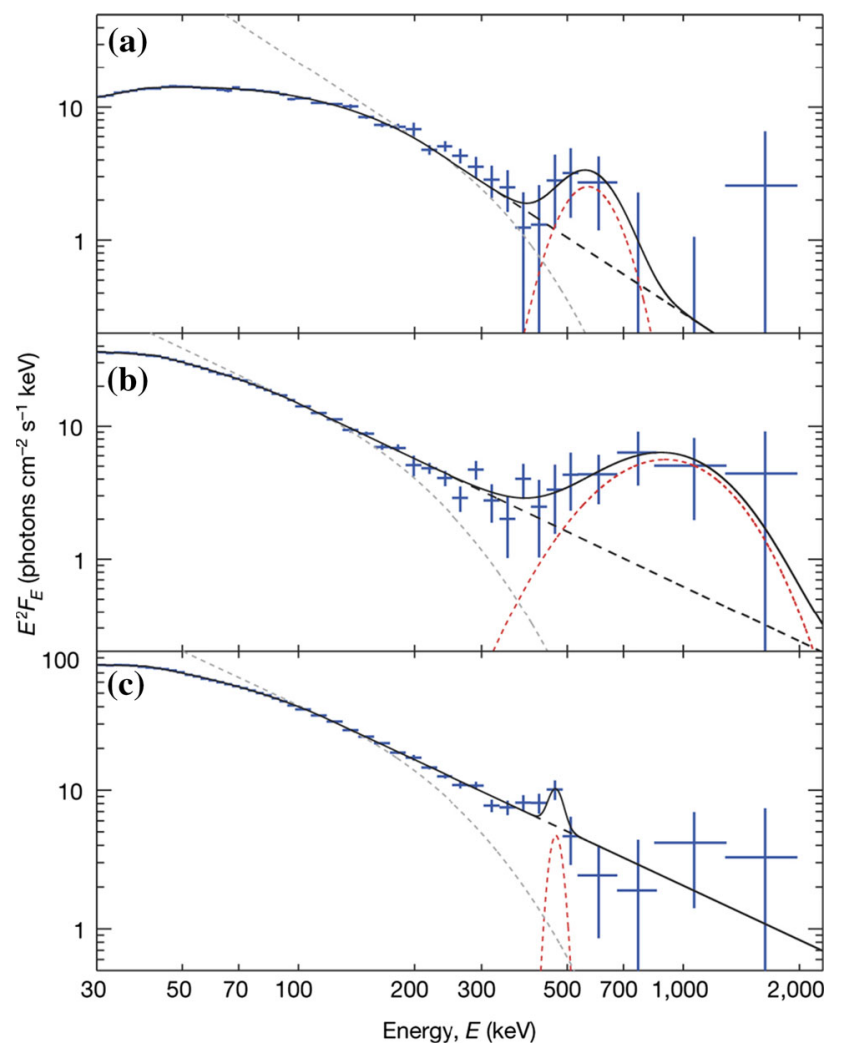

Fig. 35 Spectral evolution of V404 Cygni. INTEGRAL/SPI spectra in the soft $\gamma$-ray band in three different flaring epochs $(\mathbf{a}-\mathbf{c})$. Data (blue) are fitted as the sum of the Comptonisation continuum (black dashed curve) and annihilation radiation from a relativistic hot plasma (red dashed curve). The standard thermal Comptonisation model (grey dashed curve) fits the data up to $\sim 200 \mathrm{keV}$; it declines exponentially at higher energies and falls short of the observed flux. Our conservative, modified continuum model follows a power-law instead. [From Siegert et al. [141]]

annihilate close to their sources, and up to $40 \%$ of them escape the Galaxy, thus hinting at a source in the central region of the Galaxy.

The excess positron emission at a few hundred keV to a few MeV from V404 Cygni provides also hints towards the excess $\left(\sim 10 \mathrm{keV}\right.$ photons $\mathrm{cm}^{-2} \mathrm{~s}^{-1}$ at $1 \mathrm{MeV}$, [64]) found in the diffuse $\gamma$-ray continuum spectrum of the Galaxy's ridge emission [154]. This excess is not accounted for by models of cosmic-ray interactions with interstellar gas [64], nor by inverse-Compton emission. A few active sources at an average distance of $5 \mathrm{kpc}$ would be needed to explain the diffuse $\mathrm{MeV}$ excess. Thus, the same population of microquasars (with the same duty cycle and number of sources) could also be the origin of the observed inner Galaxy's excess in $\mathrm{MeV}$ continuum [141].

While the ACS of SPI is primarily used for vetoing events not coming through the mask, it also acts as a sensitive GRB detector (e.g. [127]). A spectacular simultaneous SPI/ACS and GROND observation was accomplished for the prompt emission of GRB 130925A, where the optical/near-infrared flux is temporally resolved into more than a dozen data points. The optical/near-infrared light curve mimics the structure in the prompt $\gamma$-rays, but is substantially delayed. Most importantly, the decay of the optical/near-infrared light decays very fast, providing the first observational evidence for a limb-brightened jet structure [61].

The INTEGRAL mission was originally planned for a duration of 3 years with a possible extension of another 2 years. But due to its excellent performance and its unique contribution to nuclear astrophysics [37] the mission has always been extended and is still in operation and performing well in the year 2021. Early players in MPE's INTEGRAL participation were V. Schönfelder, R. Diehl, R. Georgii, and C. Wunderer. A total of $21 \mathrm{PhD}$ and Master thesis were written on INTEGRAL data at MPE so far.

\subsubsection{GLAST/Fermi}

Though MPE scientists Mayer-Hasselwander, Kanbach, and Strong had been involved in the early design phase of GLAST (Gamma-ray Large Area Space Telescope; after launch renamed after Enrico Fermi) in the mid-1990s, no hardware involvement of MPE took place. However, MPE had partnered with the Marshal Space Flight Center (MSFC) to develop the Gamma-Ray Burst Monitor (GBM), with the original PI Chip Meegan (MSFC) and co-PI 
Giselher Lichti (MPE; after launch on 11 June 2008 taken over for 10 years by Jochen Greiner due to GL's retirement). GBM consists of $12 \mathrm{NaI}(\mathrm{Tl})$ scintillators read out by one PMT each and covering the $8 \mathrm{keV}-1 \mathrm{MeV}$ range, and two BGO scintillators read out by two PMTs each, covering the $200 \mathrm{keV}-40 \mathrm{MeV}$ band [114]. The $\mathrm{NaI}(\mathrm{Tl})$ are grouped in triples, looking at different directions and placed at the four corners of the spacecraft, thus allowing to monitor the full not Earth-occulted sky and derive positions of transient objects based on the count rate differences in the different detectors. The calibration of each individual detector was performed with radioactive sources (from $14.4 \mathrm{keV}$ to $4.4 \mathrm{MeV}$ ) at MPE, and additional low-energy (10-60 keV) calibration at the synchrotron radiation facility of BESSY (Germany), and high-energy calibration of the BGO detectors (4.417.6 MeV) at the SLAC National Accelerator Laboratory (USA) [16]. Subsequent system-level calibration was performed at the laboratories of the National Space Science and Technology Center (NSSTC) and of the Marshall Space Flight Center (MSFC) at Huntsville (USA).

MPE's prime science interest was in gamma-ray bursts, leaving the many other science topics (terrestrial gammaray flashes, soft gamma-repeaters, pulsars, X-ray bursts, solar flares) to our Huntsville collaborators. Bread-andbutter work has been done over the years in compiling GRB catalogues as measured with GBM, both on general properties $[17,67,166,167]$ as well as special catalogues, such as on time-resolved spectra [177] or on short-duration GRBs [26]. Special emphasis was put on creating synergy with optical/near-infrared observations with GROND (see Sect. 3.6.2), in particular with regard to unusual properties (typically higher luminosities) of Fermi/LAT GRBs [112], or those with measured redshifts which allows to study the rest-frame properties of GRBs [57,65]. Simultaneous optical observations of the prompt $\gamma$-ray emission $[45,61,66]$ provided interesting constraints on the broad-band spectral shape, the Lorentz factor, or the emission geometry. Detailed studies have been performed also on the shape of the prompt emission spectra, both on the sharpness of the peaks [176] as well as the conclusive answers provided by spectral fits with physical models $[12,24,28,175]$ rather than empirical functions (like band, or cut-off power laws). Combined GBM and POLAR measurements of GRB 170111A provided further support for a synchrotron origin by revealing a smooth polarisation angle swing in concert with a declining peak energy [27].

Another focus of investigations was to understand the large systematic errors in the GRB localisations from GBM, which could finally be understood to be of procedural nature and could be removed by the combined fitting for the spectrum and position [13,25]. An interesting application of this new analysis was our demonstration that the claimed Fermi/GBM 'emission' shortly after the binary black hole merger gravitational wave event GW 150914 was not a genuine astrophysical event and thus a chance coincidence with a sub-threshold $\gamma$-ray fluctuation [63]. In addition, a multi-year effort went into understanding the quickly varying energy-dependent background radiation as seen with the GBM detectors and reproducing it with a physically motivated multi-component model [15]. This paved the way for both, developing a pipeline for off-line transient detection [99], and measuring with unprecedented accuracy and wide coverage $(20-500 \mathrm{keV})$ the cosmic gamma-ray background as well as the Earth albedo emission [14].

An exciting event was the short-duration GRB 170817A, for which contemporaneous gravitational waves were detected with LIGO/Virgo. It was the closest known GRB detected so far and was extreme in its combination of flux, spectral softness, and temporal structure. In particular the combination of low luminosity but similar peak energy compared to standard short GRBs is difficult to explain with current models (both invoking synchrotron radiation from electrons accelerated in shocks and photospheric emission), challenging our understanding of the GRB emission process [11]. We identified a group of similar GRBs and suggested that a good fraction of the previously detected faint short GRBs is not at large redshifts, but local, at redshift smaller than 0.1, seen off-axis (Fig. 36, [23]). We incorporated off-axis emission in the estimate of the rates of short GRBs and predicted that the majority of future GW-detections of NS-NS mergers will be accompanied by faint $\gamma$-ray emission [23], contrary to previous thinking and still open for an observational test. Overall, this event was a confirmation of MPE's previous successful scientific strategy, as the only two gamma-ray measurements were taken with detectors built under MPE leadership (on INTEGRAL-SPI/ACS and Fermi/GBM), and additionally important kilonova observations could be provided with MPE's GROND imager [144].

So far, 43 of the 204 publications in refereed journals with GBM data are with MPE contribution, and $8 \mathrm{PhD}$ and Master theses have been written at MPE.

\subsection{MEGA}

In the mid-1990s, Gottfried Kanbach, together with the PhD students R. Andritschke, A. Zoglauer, P. Bloser, and F. Schopper, started the development of a next-generation telescope after COMPTEL, called MEGA, for Medium-Energy Gamma-ray Astronomy [6], see Fig. 37. The upper detector consists of a number of double-sided layers of silicon strip detectors, allowing the measurement of the track of the Compton electron. Thus, the arrival direction of the infalling gamma-ray is now restricted to a small segment on the event circle (see Fig. 2), resulting in a much better localisation and background rejection. A prototype of such a telescope [87] has been calibrated with radioactive sources and with a $100 \%$ polarised beam at the Duke University in Durham, North Carolina, at energies between 0.7 and $49 \mathrm{MeV}$, and at angles of incidence between $0^{\circ}$ and $180^{\circ}$ [179]. Such a telescope would be 


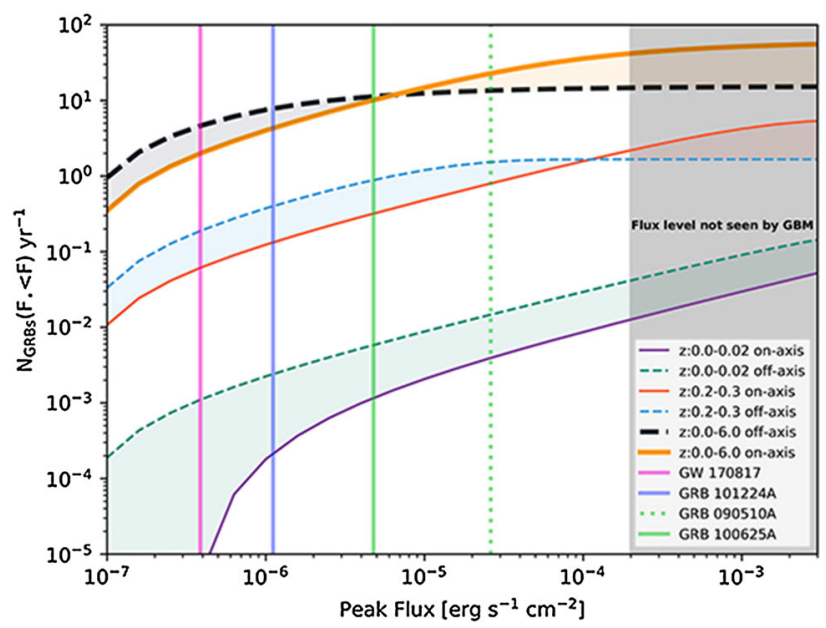

Fig. 36 The cumulative short-GRB rate distribution is shown as a function of flux, calculated by assuming a jet structure and luminosity function and then convolving this with the binary neutron star merger rate for various redshift integration ranges. The GRBs with redshift in our sample are marked as vertical lines for comparison. The dashed and solid line per redshift bin (connected with shaded area) denotes the relative frequency of detectable GRBs emitting on- vs. off-axis. At low redshift, the population of detectable short GRBs is dominated by off-axis bursts. The shaded region on the right indicates the flux values for which GBM has not (yet) observed a GRB. [From Burgess et al. [23]]

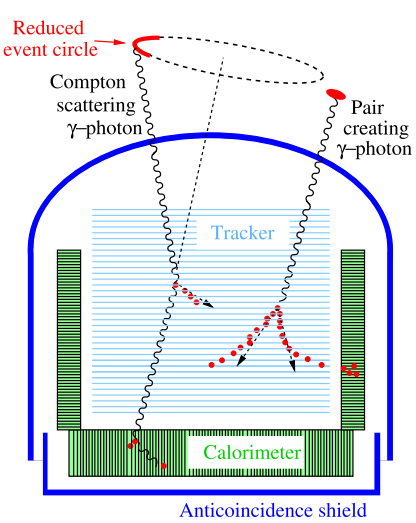

Fig. 37 MEGA, the proposed follow-up mission of COMPTEL which formed the basis for the GRIPS and ASTROGAM proposals to ESA, and AMEGO to NASA. Left: schematics of the dual-detection method [6]; middle:
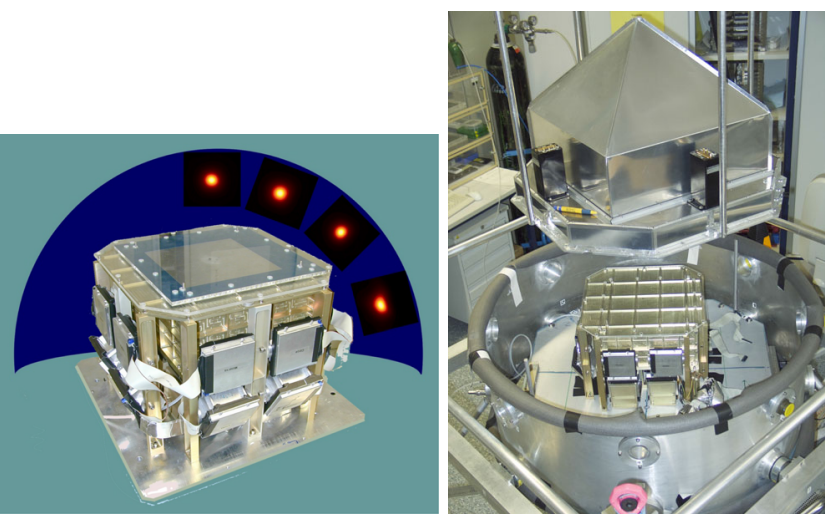

the prototype together with the reconstructed images of the $49 \mathrm{MeV}$ calibration beam at Duke University at incidence angles of $0^{\circ}, 30^{\circ}, 60^{\circ}$ and $80^{\circ}$ [5]; right: set-up for a balloon flight with anti-coincidence system [86]

about 10-times more sensitive than COMPTEL and have a 2-times better angular resolution. The telescope would also be able to detect higher energy gamma-rays up to $\sim 80 \mathrm{MeV}$ by the pair production process as indicated in Fig. 37. A proposal to DLR in 2001 for a so-called German "Kleinsatelliten" (small-satellite) mission was ranked second after the astrometry mission proposal DIVA, with ROSITA placed third. The small-satellite program was later cancelled without a single launch, leaving MEGA without any flight opportunity.

\subsection{Towards the future: hard- and software and proposals}

On 30 October 2003 the MPE Directors (R. Bender, R. Genzel, G. Hasinger, G. Morfill) decided in a memorandum the following: 1. not to develop any further MEGA hardware beyond the presently existing prototype, 2 . no substantial MPE support for a balloon flight of the existing MEGA prototype, and 3. that the other members of the existing collaboration may use the MEGA prototype for a balloon flight. With the retirement of Volker Schönfelder in October 2004, Jochen Greiner became the head of the gamma-ray group. The group resources were consequently concentrated on concept studies, development of detectors with improved properties (e.g. for Simbol-X as well as sub-nanosecond time resolution for a next-generation, post-MEGA MeV telescope), and data analysis of the missions in operation (Swift, INTEGRAL). A new focus was put on the study of gamma-ray bursts, i.e. the preparation of the Fermi/GBM flight, and the development of GROND (see below). 
Fig. 38 Basic layout of the MEGAlib software package. [From Zoglauer et al. [180]]

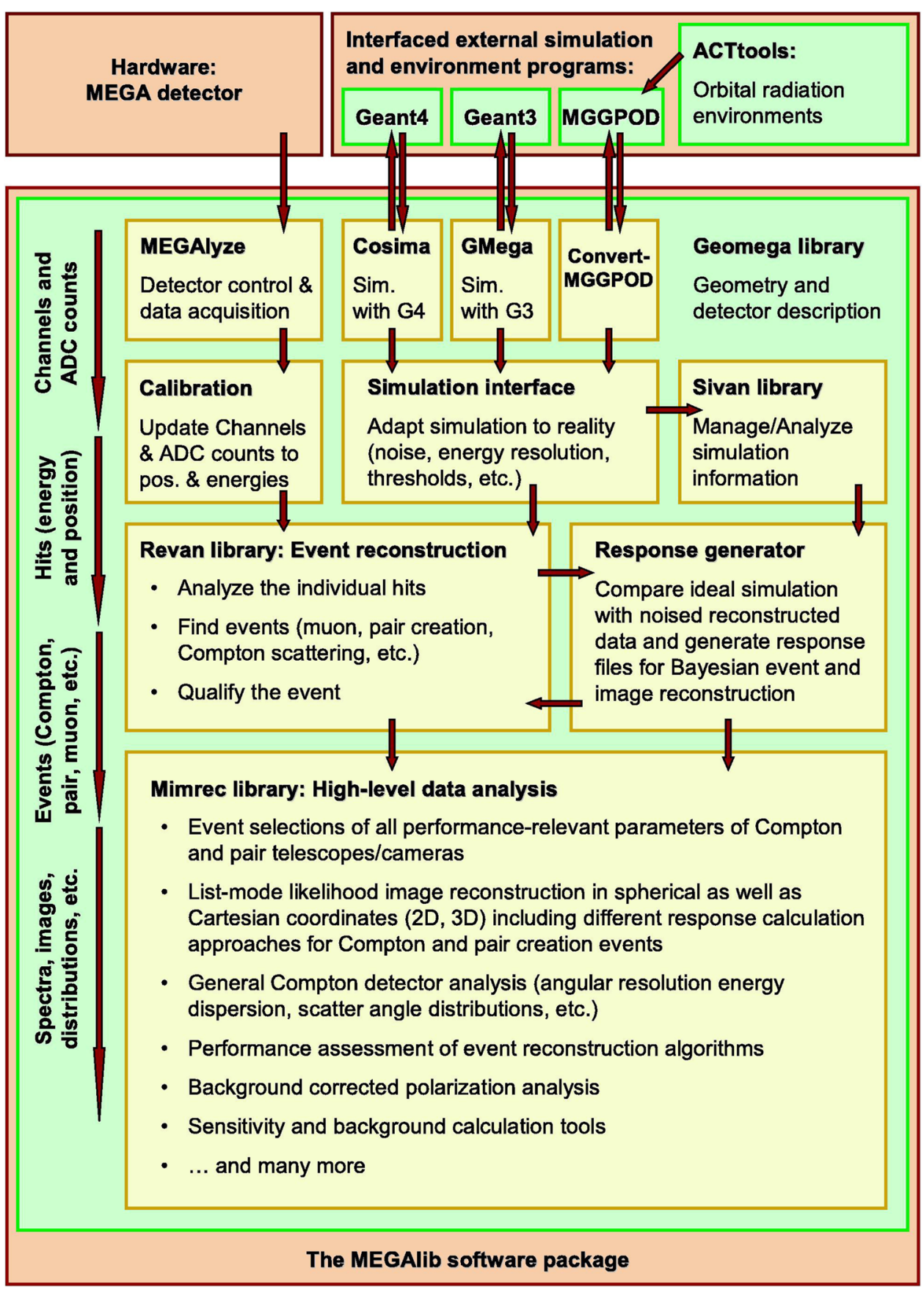

A side aspect of the MEGA effort, also with considerable impact worldwide, was the development of two software packages: MGGPOD and MEGALib. MGGPOD is a user-friendly suite of Monte Carlo codes built around the widely used GEANT package, to simulate ab-initio the physical processes relevant for the production of instrumental backgrounds [169,172]. These include the build-up and delayed decay of radioactive isotopes as well as the prompt de-excitation of excited nuclei, both of which give rise to a plethora of instrumental $\gamma$-ray background lines in addition to continuum backgrounds. MGGPOD has been used for simulating numerous instruments, among others Wind/TGRS, INTEGRAL/SPI, RHESSI, the Advanced Compton Telescope and EXIST concepts, the Brasilian MIRAX/HXI instrument, or Hitomi.

The Medium Energy Gamma-ray Astronomy Library (MEGALib) software package [178, 180] is designed to be applicable to various Compton telescope designs, including tracking, multiple Compton interactions, or timeof-flight based Compton telescopes. It encompasses the complete data analysis chain for a Compton telescope, which consists of four basic steps (Fig. 38): (a) data acquisition, either by measurements with a real detector or via simulation, (b) calibration of real data or the introduction of measurement uncertainties to the simulations, 
respectively, (c) event reconstruction, and (d) high-level data analysis including image reconstruction, polarisation analysis, sensitivity calculation, etc. MEGALib has evolved into an universal package for simulating Compton telescopes and today is used for basically every Compton instrument study.

Motivated by these improvements, both in hardware as well as software, a version even larger than MEGA, with 64 layers each consisting of a mosaic of $8 \times 8$ double-sided Si strip detectors, was proposed to ESA in 2007 within the M2 call [56] under the name GRIPS (Gamma-Ray Imaging, Polarimetry and Spectroscopy), and with slight modifications also for the M3, M4 (since then renamed Astrogam) and M5 calls [35], but so far was not selected for further consideration. MEGA and its successors also form the basis for AMEGO, the recent Astrophysics Probe mission concept of NASA. A split-off of the 2010 GRIPS version (ESA M3 proposal 2010, Greiner et al. [60]), which had focused on gamma-ray bursts and thus included an X-ray as well as near-infrared telescope with rapid slewing capability, was the THESEUS proposal [4] for ESA's M5 mission, which also was not selected for flight.

With the arrival of the new director for the X-ray and gamma-ray group in 2010, Kirpal Nandra, the MPE support for all gamma-ray activities was stopped, and INTEGRAL and Fermi science left as formally undesired projects to the enthusiasm of few individuals.

\subsection{Complementary low-energy instruments}

\subsubsection{High time resolution studies with OPTIMA}

The OPtical TIMing Analyzer (OPTIMA), initiated by Gottfried Kanbach, is a portable, stand-alone high-speed photopolarimeter $[88,148]$. Originally designed for observations of pulsars, it has found exciting applications in other highly time-variable sources like magnetars, cataclysmic variables, or black hole X-ray binaries. OPTIMA records the arrival times of individual optical photons passing either through a fibre unit for photometry or a twin Wollaston prisms for polarimetry. The single photon-timing accuracy is $5 \mathrm{~ns}$ relative to a GPS clock. It operates in the 450-900 $\mathrm{nm}$ range and reaches about 20th mag in $1 \mathrm{~s}$ exposure at a 2-3 $\mathrm{m}$ class telescope. OPTIMA has been operated successfully in visitor-mode at the following observatories: $1.3 \mathrm{~m}$ Skinakas (Greece), $1.9 \mathrm{~m}$ SAAO (Australia), 2.2 m MPG/ESO La Silla (Chile), $2.56 \mathrm{~m}$ NOT La Palma, and $3.5 \mathrm{~m}$ Calar Alto (Spain).

Among the first highlights was the coordinated observation of the black-hole X-ray binary XTE J1118+480 with RXTE, which revealed strong and puzzling correlations between the optical and X-ray emission [85]. While the optical light grossly follows the X-rays, the most puzzling feature of the cross-correlation is a dip at $2-5 \mathrm{~s}$ before zero lag. This drop of the optical brightness before the onset of an X-ray increase poses a challenge for any model of the optical emission which in the simplest picture is due to reprocessing of the X-ray emission.

The linear polarisation of the Crab pulsar was measured at $11 \mu \mathrm{s}$ time resolution, providing about 3000 phase intervals per pulsar rotation. The degree of optical polarisation and the position angle correlate in surprising details with the optical and radio light curves. Different models can reproduce some of the observed features (Fig. 39), but none provides a fully convincing explanation for all features, thus requiring more elaborate theoretical models $[78,143]$.

We know rapid, second-scale X-ray flares from X-ray bursters, and gamma-ray flares from GRBs or Soft Gamma Repeaters (SGR). When the Swift satellite triggered on a new transient (in fact originally mistaken for a GRB), OPTIMA observations started within a few minutes and followed the evolution of the optical counterpart of this transient (SWIFT J195509.6+261406). Surprisingly, extremely bright and rapid optical flaring was seen (Fig. 40, [146]). The shortest timescales of $0.3-0.4 \mathrm{~s}$ correspond to a maximum size of the emitting region of $\sim 10^{10} \mathrm{~cm}$. The flares have maximum extinction-corrected I-band isotropic luminosities of $1-2 \times 10^{35}(\mathrm{~d} / 5 \mathrm{kpc})^{2} \mathrm{erg} \mathrm{s}^{-1}$. Combined with the above size limit, thermal emission is very unlikely to cause these optical flares. The optical flares are phenomenologically similar to high-energy flares of SGRs, which are thought to be neutron stars with extremely high magnetic fields (magnetars). This suggests similar processes in operation, but with the peak of the emission at lower frequencies. Unfortunately, only little simultaneous X-ray coverage was available, making it impossible to check for a strict correlation of optical and X-ray flaring vs. flaring where some physical parameter changes the peak energy of the emission.

\subsubsection{GRB afterglow studies with GROND}

The primary goal of building the GRb Optical/Nearinfrared Detector GROND [55] was to rapidly identify GRB afterglows and measure their redshift. This led to the concept of a camera which allows simultaneous observations in multiple filters throughout the optical and near-infrared (NIR) region. The simultaneity is dictated by the fact that a typical GRB afterglow initially fades by about 2-3 mag within 5-10 min after the GRB, and by another 3 mag in the following $50 \mathrm{~min}$. Together with frequent deviations from a plain power-law decay, this renders cycling through different filters useless. Furthermore, with the advent of Swift's detection of $\sim 100$ GRBs/year, follow-up of each GRB with an $8 \mathrm{~m}$ telescope became impractical, and some knowledge-based pre-selection was needed. Four bands were implemented in the visual, plus three (standard $J H K_{s}$ ) bands in the near-infrared (NIR). The 

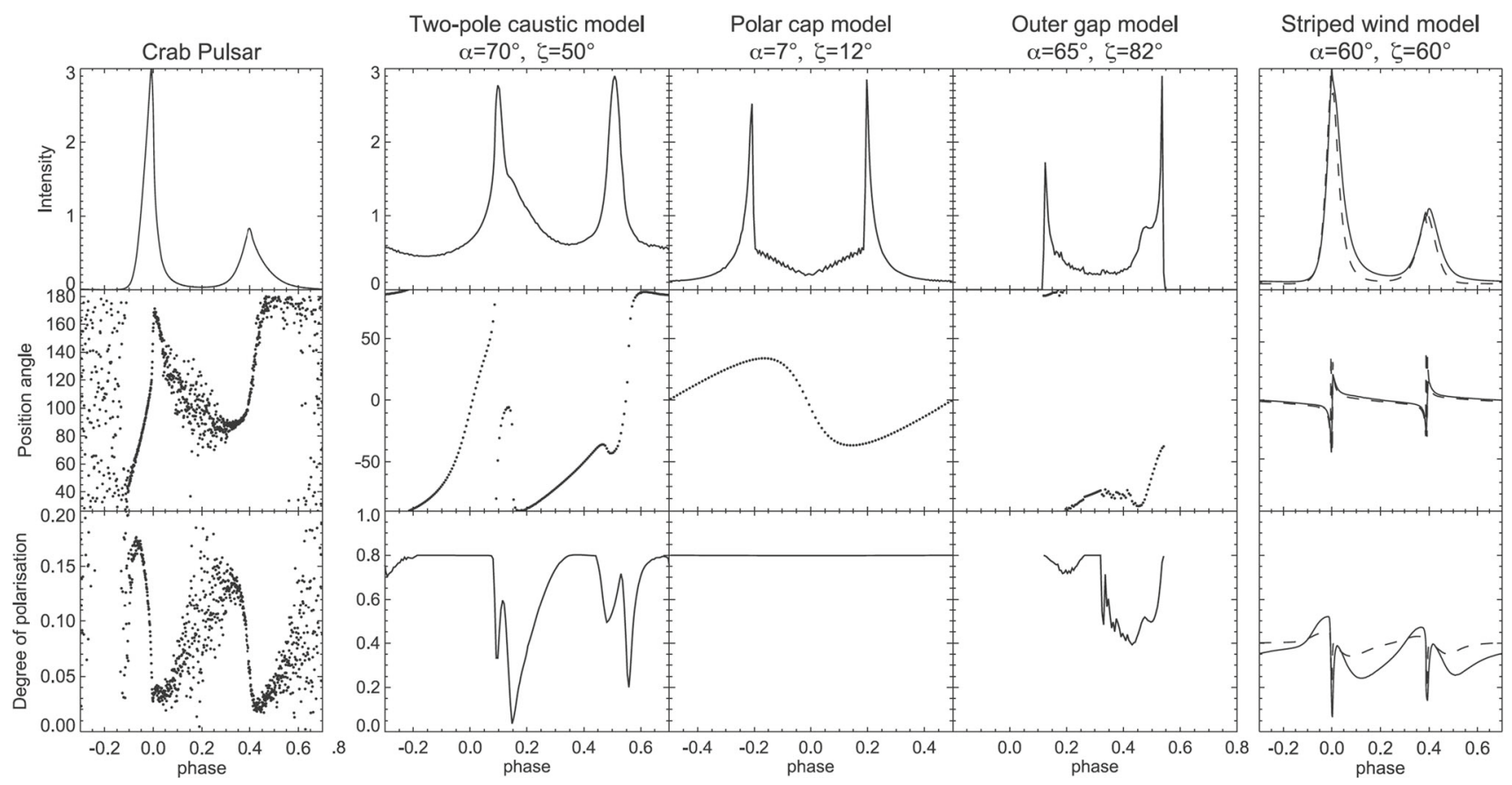

Fig. 39 Comparison between observations and models for the optical polarisation of the Crab pulsar. The left-hand column shows (DC subtracted) the light curve (Stokes I), position angle, and polarisation degree as a function of pul-

sar phase (from top to bottom, respectively). The other four columns show the predictions of four models. [From Slowikowska et al. [143]]

Fig. 40

OPTIMA-measured light curve of Swift

$\mathrm{J} 195509.6+261406$ at $4 \mu \mathrm{s}$ resolution, rebinned at 10 $\mathrm{s}$, showing the unique optical variability. [From Stefanescu et al. [146]]

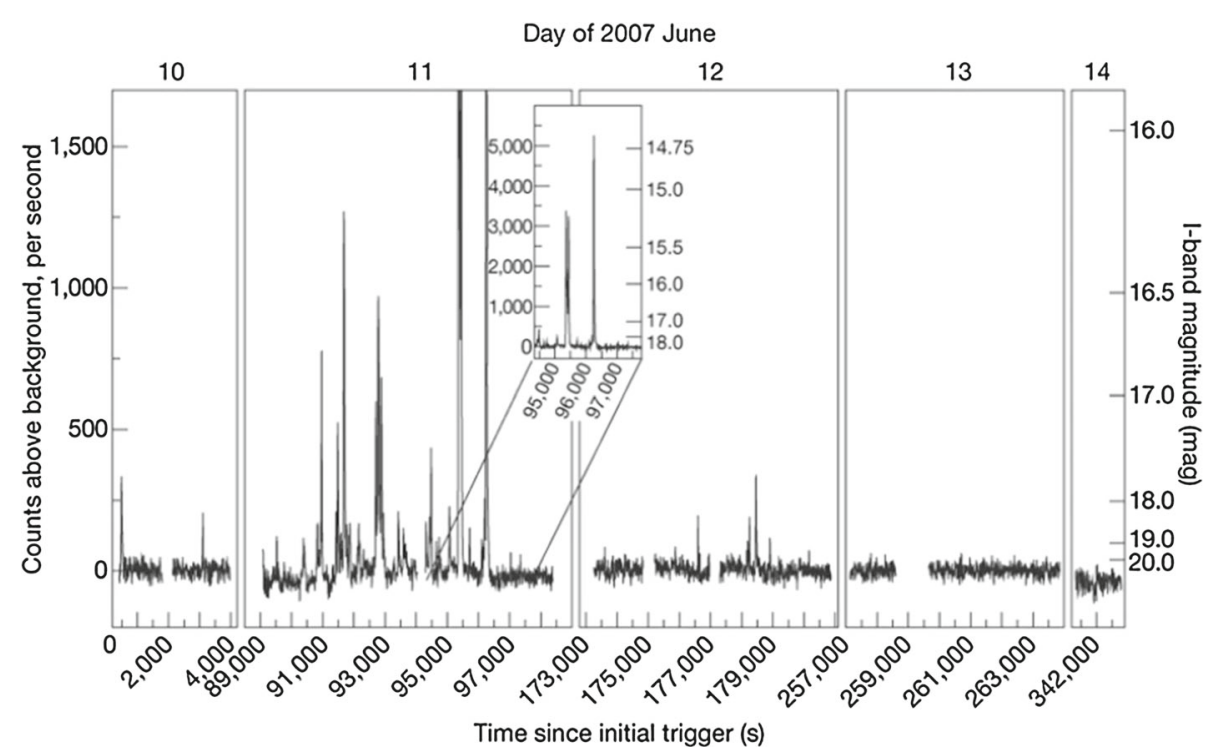

separation of the different photometric bands was achieved using dichroic beamsplitters in the converging beam (Fig. 41). More technical details can be found in Greiner et al. [55]. GROND was commissioned at the MPG 2.2 m telescope at La Silla (ESO, Chile) in April/May 2007, and a routine GRB follow-up program was performed until its termination in Oct. 2016. During this time, over 500 GRB afterglows were observed, among those $82 \%$ of all Swift-detected GRBs observable from Chile.

One year after the commissioning, the afterglow of GRB 080913 at $z=6.7$ (see Fig. 42) was discovered with GROND [58] and spectroscopically confirmed with ESO/VLT spectroscopy, thus serving as the "proof-of-concept" for using simultaneous multi-band photometry to accurately measure photometric redshifts of GRBs. Another early and surprising result was the very dusty GRB 070802 at a redshift of $z=2.45$. The SED deviated clearly from the typical synchrotron power-law shape, showing increasing curvature towards the bluest band and a lowflux 'outlier' in the $i^{\prime}$-band (Fig. 43). We interpreted the $i^{\prime}$-band drop as the $2175 \AA$ extinction feature in the GRB 
Fig. 41 Scheme of the optical beam path of GROND with the optical components and the detectors labelled. [From Greiner et al. [55]]
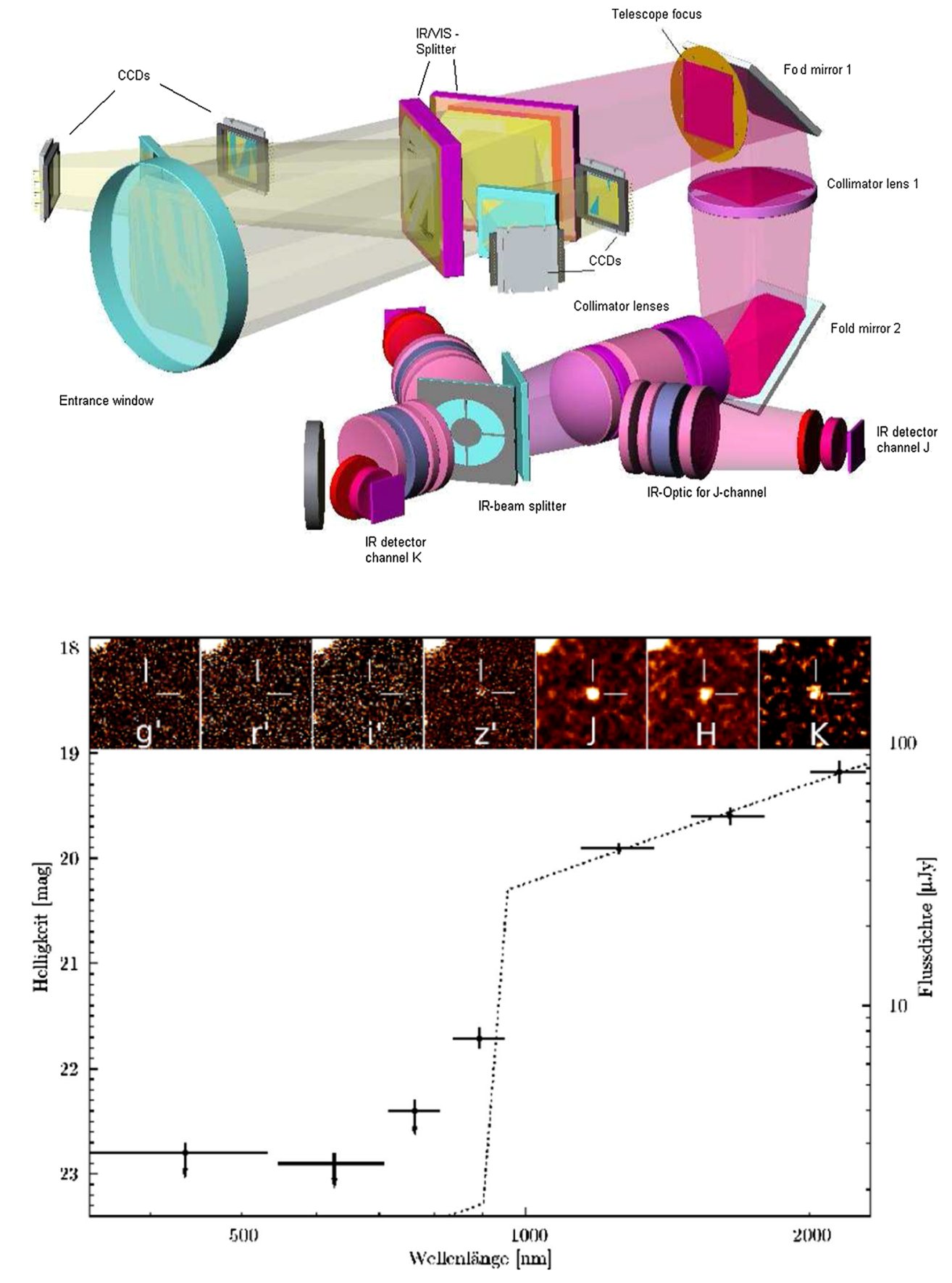

Fig. 42 GROND spectral energy distribution and the corresponding image cut-outs (top row) for GRB 080913 at $z=6.7$ [58]. The GROND observation started about 6 min after the Swift/BAT trigger, and the

photometric redshift was available $35 \mathrm{~min}$ after the trigger. This information was used to trigger FORS spectroscopy at ESO/VLT which confirmed the GROND photo- $z$ to be accurate to within $5 \%$ [From Greiner et al. [58]]

host galaxy [98]. This was one of the first and clearest detections of the $2175 \AA$ feature at high redshift and was later confirmed by optical spectroscopy [44] with VLT/X-shooter.

The biggest impact of GROND observations of GRBs was undoubtedly the initiation of systematic afterglow follow-up in the near-infrared $\left(J H K_{s}\right)$, enabling (i) the discovery of a larger fraction of high-redshift GRBs as well as (ii) studying the prevalence of dust along GRB sightlines, i.e. quantifying the incidence of 'dark' bursts [59]. Other scientific highlights include (iii) studies of Fermi-detected GRBs [112] (including the redshift estimate of GRB 080916C), (iv) measurements of the SEDs of non-canonical light curve variability such as flares or intensity jumps, (v) the discovery and detailed study of a large fraction of all GRB-SNe, most prominently the SN 2011kl related to the ultra-long GRB 111209A [62], (vi) tests of the simplest fireball scenario based on the evolution of afterglow SEDs, (vii) the investigation of the jet structure and/or off-axis viewing geometry based on achromatic afterglow light curves, and (viii) the characterisation of about half of all optically detected short GRB afterglows [117]. An overview on non-GRB science with GROND is given in Greiner [53]. Over the years, $26 \mathrm{PhD}$ and Master 
Fig. 43 GROND $g^{\prime} r^{\prime} i^{\prime} z^{\prime} J H K_{s}$ spectral energy distribution of the afterglow of GRB 070802, showing a clear drop of the $i^{\prime}$-band, interpreted as the redshifted $2175 \AA$ bump in the GRB host galaxy [From Krühler et al. [98]]

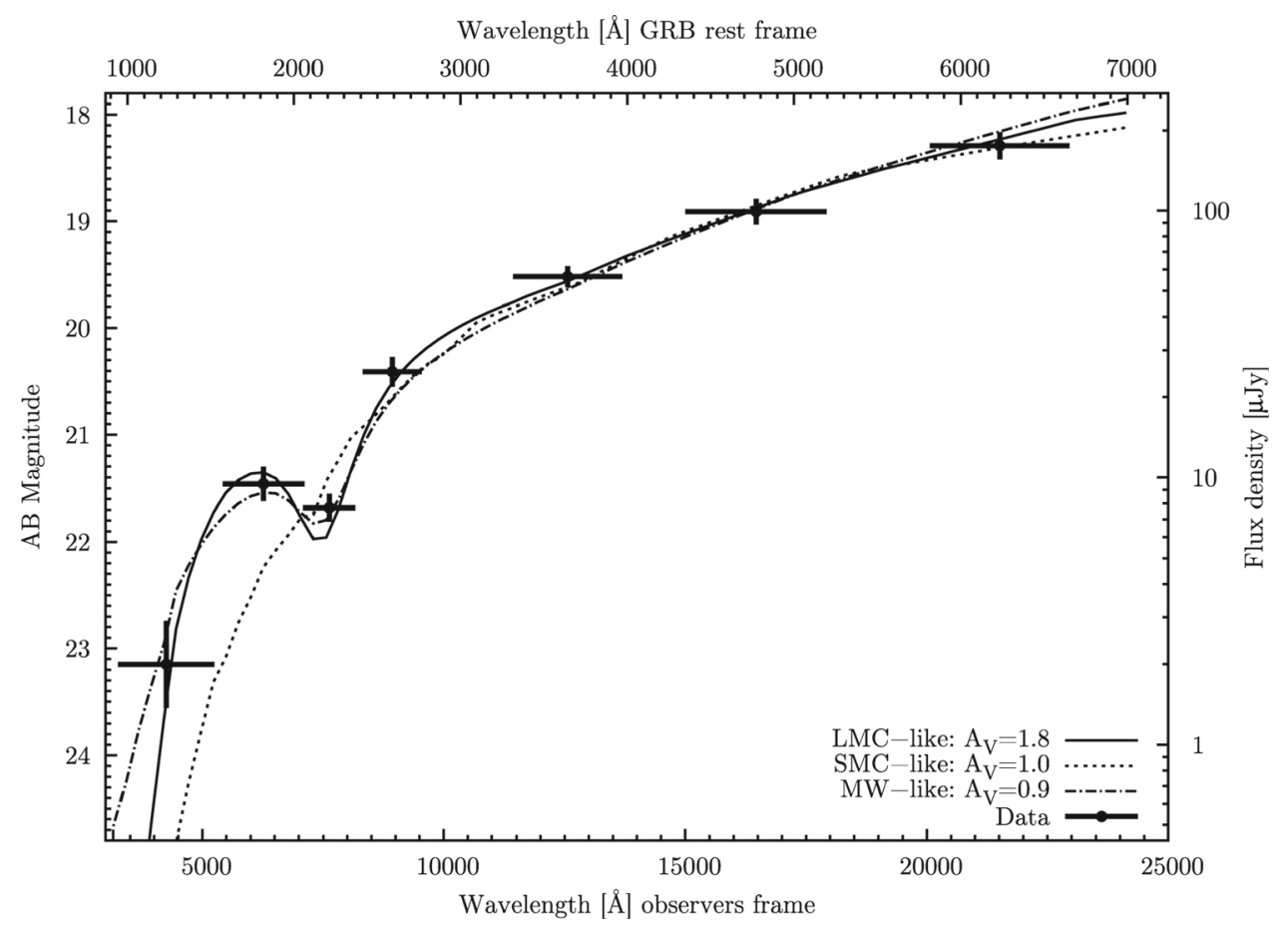

theses were written using GROND data. Upon directorial decision, the systematic GRB afterglow program with GROND was terminated in October 2016.

\section{Conclusions}

During the last 50-60 years, gamma-ray astronomy has been explored as a new field of astronomy, and spectacular discoveries have been made. First, complete sky surveys have been accomplished in the 1990's by the two instruments EGRET and COMPTEL aboard the Compton Gamma-Ray Observatory of NASA. We have learnt that the sky is rich in high-energy phenomena. It was found that the most compact and energetic objects are gamma-ray emitters. These are neutron stars, stellar and massive black holes, SN explosions and their remnants, and cosmic rays via their interactions with matter and fields. In addition it was found that part of the gamma-ray sky is continuously changing. With gamma-rays we see the most violent and energetic parts of the Universe. It has been recognised that objects exist in the Universe like pulsars, gamma-ray blazars, and the famous gamma-ray burst sources which all have their maximum of luminosity in the gamma-ray range. It is practically impossible to understand these objects without knowing their gamma-ray properties. Highlights with a leading role of MPE include:

- the invention of the Compton telescope for MeV gamma-ray astronomy [137]

- the measurement of the angular distribution of the atmospheric neutron flux with the 1971 Palestine balloon flights, confirming the origin of the high-energy protons in the Earth's inner radiation belt due to decaying neutrons [81],

- the first comprehensive characterisation of the Vela pulsar in gamma-rays with COS-B [82],

- the large-scale distribution of gamma radiation along the Galactic disk observed by COS-B [109],

- the latitude-dependent distribution of the total gas column density as measured with COS-B and its relation to the molecular-hydrogen column density [151],

- the discovery of a 154-day periodicity in the occurrence of hard solar flares [128],

- the first gamma-ray detection of Cen-A with the Uberaba balloon flight [164],

- EGRET measurements up to $1 \mathrm{GeV}$ of the spectacular long solar gamma-ray flare on 11 June 1991 [83],

- the first detailed pulse profile measurements of the Vela pulsar with EGRET [84],

- the discovery of ${ }^{44} \mathrm{Ti}$ emission from Cas-A with COMPTEL [76],

- the first all-sky continuum map in the 1-30 MeV range with COMPTEL containing emission from point sources and extended regions [132],

- the prove that the MeV bump in the diffuse cosmic background spectrum discovered by Apollo XV was an artefact caused by background radiation $[89,168,170]$ 
- the measurement of high-energy gamma-ray emission from the Galactic Center with EGRET [111],

- an improved propagation of cosmic-ray nucleons in the Galaxy [150],

- the study of MeV emission from the nearest AGN, Cen A, with COMPTEL [147],

- the first catalogue of MeV sources from COMPTEL [133,140],

- the correlated fast X-ray and optical variability found in the black-hole binary XTE J1118+480 with OPTIMA [85],

- the GALPROP model for diffuse Galactic continuum gamma-rays $[152,153]$,

- the dissection of the spatial and kinematic properties of the $1.8 \mathrm{MeV}{ }^{26} \mathrm{Al}$ line with INTEGRAL [40], preceded by the first $1.8 \mathrm{MeV}$ all-sky map from COMPTEL [39,118,123],

- the detailed mapping of the Galactic $511 \mathrm{keV}$ emission from the Galactic bulge [173,174],

- the measurement of cosmic X-ray background and Earth albedo spectra with Swift BAT[2],

- the discovery of ultra-fast optical flaring from a Galactic transient, a likely magnetar, with OPTIMA [146],

- the improved measurements of high redshifts of GRBs via the initiation of systematic NIR observations with GROND [58],

- the surprising spin-resolved optical polarisation of the Crab with OPTIMA [143],

- the finding of a high fraction of Compton-thick AGN with a hard X-ray survey using Swift/BAT [29],

- the clarification of the nature of dark GRBs with GROND [59],

- the unusual early ${ }^{56} \mathrm{Ni}$ decay gamma-rays from supernova SN2014J with INTEGRAL [41],

- the evidence for a magnetar origin of ultra-long GRBs with the GROND discovery of a supernova (SN 2011kl) related to GRB 111209A [62],

- the discovery of strong $511 \mathrm{keV}$ positron annihilation emission in the outburst of the microquasar V404 Cyg with INTEGRAL [141].

The large number of spectacular discoveries in gamma-ray astronomy will keep astronomers and astrophysicists busy for many years to come. The Max-Planck Institute for Extraterrestrial Physics has significantly contributed to the development of gamma-ray astronomy during the last half century.

Acknowledgements While we have made extensive cross-checks with our colleagues, this is a subjective review, and other people might have different re-collections or interpretations of the historical events. We acknowledge helpful discussions with Klaus Pinkau, Joachim Trümper, Günther Hasinger, Roland Diehl, Gottfried Kanbach, and Giselher Lichti. We thank Werner Collmar for providing an electronic version of Fig. 26. JG is very grateful for Bettina Niebisch's major extension work in Fig. 3 and the correction of the Compton mode sub-panel in Fig. 2.

Funding Open Access funding enabled and organized by Projekt DEAL.

Open Access This article is licensed under a Creative Commons Attribution 4.0 International License, which permits use, sharing, adaptation, distribution and reproduction in any medium or format, as long as you give appropriate credit to the original author(s) and the source, provide a link to the Creative Commons licence, and indicate if changes were made. The images or other third party material in this article are included in the article's Creative Commons licence, unless indicated otherwise in a credit line to the material. If material is not included in the article's Creative Commons licence and your intended use is not permitted by statutory regulation or exceeds the permitted use, you will need to obtain permission directly from the copyright holder. To view a copy of this licence, visit http://creativecommons.org/licenses/by/4.0/.

\section{References}

1. Abdo, A.A., Ackermann M., Ajello, M. et al. 2010, A\&A 523, A46

2. Ajello M., Greiner J., Sato G. et al. 2008, ApJ 689, 666

3. Allen G.E., Chow K., DeLaney T., et al. 2014, ApJ 798, 82

4. Amati L., O’Brien P., Götz D., et al. 2018, Adv. Space Res. 62, 191

5. Andritschke R., Zoglauer A., Kanbach G. et al., 2004, in Proc. 5th INTEGRAL Workshop, Feb. 2004, Munich, Eds. V. Schönfelder, G. Lichti \& C. Winkler, ESA SP-552, p. 761

6. Andritschke R., et al., 2005, Exp. Astron. 20, 395

7. Aschenbach, B., 1998, Nature 396, 141

8. Bai T., Cliver E.W., 1990, ApJ 363, 299

9. Ballester J.L., Oliver R., Carbonell M., 2002, ApJ 566, 505

10. Band D., Matteson J., Ford L., et al. 1993, ApJ 413, 281

11. Begue D., Burgess J.M., Greiner J., 2017, ApJ 851, 19

12. Berlato F., Burgess J.M., Greiner J. et al. 2021, A\&A (subm.)

13. Berlato F., Greiner J., Burgess J.M., 2019, ApJ 873, 60 
14. Biltzinger B., et al. 2022, A\&A (subm)

15. Biltzinger B., Kunzweiler F., Greiner J., Toelge K., Burgess J.M., 2020, A\&A 640, 8

16. Bissaldi E., von Kienlin A., Lichti, G., et al. 2009, Exp. Astron. 24, 47

17. Bissaldi E., von Kienlin A., Kouveliotou C., et al. 2011, ApJ 733, 97

18. Bloemen, H. et al., 1995, A\&A 293, L 1

19. Blom, H., et al., 1995, A\&A 298, 233

20. Bothe, W., Kolhörster, W., 1929, Zeitschrift f. Physik 56, 751

21. Brecher K., Burbidge G.R., 1972, ApJ 174, 253

22. Briggs M.S., Band D.L., Kippen R.M., et al. 1999, ApJ 524, 82

23. Burgess J.M., Greiner J., Begue D. et al., 2017 arXiv:1710.05823, after massive editorial delays finally published in Frontiers Astron. Sp. Sci. 7 (2020), 40

24. Burgess J.M., 2019, A\&A 629, 69

25. Burgess J.M., Yu H.-F., Greiner J., Mortlock D., 2018, MN 476, 1427

26. Burgess J.M., Greiner J., Begue D., Berlato F., 2019a, MN 490, 927

27. Burgess J.M., Kole M., Berlato F., et al. 2019b, A\&A 627, 105

28. Burgess J.M., Begue D., Greiner J. et al. 2020, Nature Astron. 4, 174

29. Burlon, D., Ajello, M., Greiner, J. et al. 2011, ApJ 728, 58

30. Caraveo P.A., Bennett K., Bignami G.F., et al. 1980, A\&A 91, L3

31. Cerviño M., Diehl R., Kretschmer K., Plüschke S. 2002, New Astron. Rev. 46, 541

32. Chupp, E.L. et al, 1973, Nature 241, 333

33. Clark G.W., et al. 1968, ApJ 153, L203

34. Collmar, W. et al., 1999, Astrophs. Letters \& Communications 38, 445

35. De Angelis, A., Tatischeff, V., Tavani, M., et al. 2017, Exp. Astron. 44, 25

36. di Mauro, M., 2018, in 14th Marcel Grossmann Meeting, July 2015, Rome, Eds. M. Bianchi et al., World Scientific Publ. ISBN \#9789813226609, p. 3098

37. Diehl R., Hartmann D.H., Prantzos N., 2018a, "Astrophysics with radioactive Isotopes", Astrophysics and Space Science Library 453, Springer (https://doi.org/10.1007/978-3-319-91929-4)

38. Diehl R., Timmes F.X, 1998, PASP 110, 637

39. Diehl R., Dupraz C., Bennett K., et al. 1995, A\&A 298, 445

40. Diehl R., Halloin H., Siegert T. et al. 2006, Nature 439, 45

41. Diehl R., Siegert T., Hillebrandt W. et al. 2014, Science 345, 1162

42. Diehl R., Siegert T., Hillebrandt W. et al. 2015, A\&A 574, A72

43. Diehl R., Siegert T., Greiner J. et al. 2018b, A\&A 611, A12

44. Eliasdóttir Á., Fynbo J.P.U., Hjorth J., et al. 2009, ApJ 697, 1725

45. Elliott J., Yu H.-F., Schmidl S., et al. 2014, A\&A 562, A100

46. Fishman G.J., 1972, ApJ 171, 163

47. Forrest, D.J. et al, 1980, Solar Physics 65, 15

48. Gehrels, N., Fichtel C.E., Fishman G.J., Kurfess J.D., Schönfelder V., 1993, Sci. American 269 (Dec. 1993$), 68$

49. Giacconi R., Gursky H., Paolini F.R., Rossi B.B., 1962, PRL 9, 439

50. Ginzburg V.L., 1972, Nature 239, 8

51. Graser, U., Schönfelder, V., 1977, J. Geophys. Res. 82, 1055

52. Graser, U., Schönfelder, V., 1982, ApJ 263, 677

53. Greiner J., 2019, PASP 131, 015002

54. Greiner J., Sommer M., Bade N. et al. 1995, A\&A 302, 121

55. Greiner J., Bornemann W., Clemens C., et al. 2008, PASP, 120, 405

56. Greiner J., Iyudin, A., Kanbach, G., et al. 2009a, Exp. Astron. 23, 91

57. Greiner J., Clemens C., Krühler T., et al. 2009b, A\&A 498, 89

58. Greiner J., Krühler T., Fynbo J.P.U, et al. 2009c, ApJ, 693, 1610

59. Greiner J., Krühler T., Klose S. et al., 2011, A\&A, 526, A30

60. Greiner J., Mannheim K., Aharonian F., et al. 2012, Exp. Astron. 34, 551

61. Greiner J., Yu H.-F., Krühler T., et al. 2014, A\&A 568, A75

62. Greiner J., Mazzali P.A., Kann D.A. et al. 2015, Nature 523, 189

63. Greiner J., Burgess J.M., Savchenko V., Yu H.-F., 2016, ApJ 827, L38

64. Grenier I.A., Black J.H., Strong A.W., 2015, ARA\&A 53, 199

65. Gruber D., Greiner J., von Kienlin A. et al. 2011a, A\&A 531, A20

66. Gruber D., Krühler T., Foley S. et al. 2011b, A\&A 528, A15

67. Gruber D., Goldstein A., Weller von Ahlefeld V. et al. 2014, ApJS 211, 12

68. Hartmann, R.C. et al., 1999, ApJS 123, 79

69. Hayakawa, S., 1952, Prog. Theor. Phys. 8, 571

70. Haymes, R.C., et al. 1968, ApJ 151, L12

71. Herterich, K., Pinkau, K., Rothermel, H., Sommer, M., 1973, in Proc. 13th Int. Cosmic Ray Conf., Denver, vol 1 (OG sessions), p. 21

72. Hess, V., 1912, Physikal. Zeitschrift, 13. Jahrgang, No 21/22, 1. Nov. 1912, S. 1084-1091 
73. Hurley K., Sommer M., Atteia J.-L., et al. 1992, A\&A Suppl. 92, 401

74. Hurley K., Dingus, B.L., Mukherjee, R. et al. 1994, Nature 372, 652

75. Hutchinson, G.W., 1952, Phil. Mag. 43, 847

76. Iyudin, A.F. et al., 1994, A\&A 284, L1

77. Iyudin, A.F. et al., 1998, Nature 396, 142

78. Kanbach G., Slowikowska A., Kellner S., Steinle H., 2005a, in "Astrophysical Sources of High Energy Particles and Radiation", eds. T. Bulik et al., AIP Conf. Proc. 801, 306

79. Kanbach, G., Schönfelder, V., Zehnder, A., 2010, in "Observing Photons in Space", eds. M.C.E. Huber et al., ISSI Sci Rep. Ser., ISBN 978-92-9221-938-8, p. 57

80. Kanbach, G., et al., 1988, SSR 49, 69

81. Kanbach, G., Reppin, C. ; Schönfelder, V., 1974, J. Geophys. Res. 79, 5159

82. Kanbach, G., Bennett, K., Bignami, G.F., et al., 1980, A\&A 90, 163

83. Kanbach G., Bertsch D.L., Fichtel C.E., et al. 1993, A\&A Suppl. 97, 349

84. Kanbach G., Arzoumanian Z., Bertsch D.L., et al. 1994, A\&A 289, 855

85. Kanbach G., Straubmeier C., Spruit H.C., Belloni T., 2001, Nature 414, 180

86. Kanbach G., Andritschke R., Schopper F., et al. 2004, New Astron. Rev. 48, 275

87. Kanbach G., Andritschke R., Zoglauer A., et al. 2005b, NIM A541, 310

88. Kanbach G., Stefanescu A., Duscha S., 2008, ASSL 351, 153

89. Kappadath, S.C., et al, 1997, in Proc. of 4th Compton Symp., AIP Conf. Proc 410, 1218

90. Kappadath, S.C., et al, 1996, A\&AS 120, 619

91. Kniffen, D.A., Fichtel, C.E., 1981, ApJ 250, 389

92. Kolhörster, W., 1913, Physikal. Zeitschrift No 22/23, 14. Jahrgang, 15.11.1913, S. 1153-1156

93. Krause M.G.H., Diehl R., Bagetakos Y. et al. 2015, A\&A 578, A113

94. Kraushaar, W.L., et al., 1965, ApJ 141, 845

95. Kraushaar, W.L., et al., 1972, ApJ 177, 341

96. Kretschmer K., Diehl R., Krause M.G.H. et al. 2013, A\&A 559, A99

97. Krivova N.N., Solanki S.K., 2002, A\&A 394, 701

98. Krühler T., Küpcü Yoldaş A., Greiner J., et al. 2008, ApJ 685, 376

99. Kunzweiler F., Biltzinger B., Greiner J., Burgess J.M., 2021, A\&A

100. Kurfess J., 1992, ApJ, 399, L137

101. Leising, M.D., Share, G. H., 1990, ApJ 357, 638

102. Leonard P.J., Gehrels N., 2009, https://heasarc.gsfc.nasa.gov/docs/history, version 1.0.8

103. Lichti, G.G., Bennett K., den Herder J.W. et al. 1994, A\&A 292, 569

104. Lichti, G.G., Balonek, T., Courvoisier, T. et al. 1995, A\&A 298, 711

105. Mahoney W.A., Ling J.C., Jacobson A.S., Lingenfelter R., 1982, ApJ 262, 742

106. Martin P., Strong A.W., Jean P., Alexis A., Diehl R., 2012, A\&A 543, A3

107. Mayer-Hasselwander, H.A., et al., 1972, ApJ 175, L23

108. Mayer-Hasselwander, H.A., Bennett K., Bignami G.F., et al., 1980, Ann. NY Acad. Sci. 336, 211

109. Mayer-Hasselwander, H.A., Bennett K., Bignami G.F., et al., 1982, A\&A 105, 164

110. Mayer-Hasselwander H.A., Bertsch D.L., Brazier K.T.S., et al. 1994, ApJ 421, 276

111. Mayer-Hasselwander H.A., Bertsch D.L., Dingus B.L., et al. 1998, A\&A 335, 161

112. McBreen S., Krühler T., Rau A. et al. 2010, A\&A 516, A71

113. McConnell M.L., Zdziarski A.A., Bennett K., et al. 2002, ApJ 572, 984

114. Meegan C., Lichti G., Bhat P.N., et al. 2009, ApJ 702, 791

115. Millikan R.A., Cameron G.H., 1928, PRL 32, 533

116. Morrison, P., 1958, Nuovo Cimento 7, 858

117. Nicuesa Guelbenzu A., Klose S., Greiner J., et al. 2012, A\&A 548, A101

118. Oberlack, U., et al., 1996, A\&AS 120, 311

119. Peterson L., et al., 1966, in Proc. of 6th Int. Sp. Sci. Symp., Space Res. VI, ed. R.L. Smith-Rose, Spartan Books, p. 53 (ASIN: B000KFUA8W)

120. Pinkau, K. 1966, Zeitschr. f. Naturforschung 21a, 2100

121. Pinkau, K., 1996, A\&AS 120, 43

122. Pinkau, K., 2009, Exp. Astron. 5, 157

123. Plüschke S., Diehl R., Schönfelder V., et al. 2001, In: "Exploring the gamma-ray Universe", Proc. of the Fourth INTEGRAL Workshop, 4-8 September 2000, Alicante, Spain. Editor: B. Battrick, ESA SP-459, p. 55-58

124. Plüschke S., Cerviño M., Diehl R., Kretschmer K., Hartmann D.H., Knödlseder J., 2002, New Astron. Rev. 46,535

125. Prantzos N., Diehl R., 1996, Phys. Rep. 267, 1

126. Rank, G. et al., 2001, A\&A 378, 1046

127. Rau A., Kienlin A.V., Hurley K., Lichti G.G., 2005, A\&A 438, 1175

128. Rieger E., Share G.H., Forrest D.J., et al. 1984, Nature 312, 623

129. Rieger E., Vestrand W.T., Forrest D.J., et al. 1989, Science 244, 441

130. Rossi B., 1964, "Cosmic Rays", Mc Graw-Hill https://web.mit.edu/8.13/8.13c/references-fall/muons/ rossi-cosmic-rays-1964.pdf 
131. Rossi B., 1965, in "Space Research V", Proc. 5th Int. Space Sci. Symp., Florence, May 12-16, 1964, Eds. D.G. King-Hele, P. Muller and G. Righini, North-Holland Publ. Comp., Amsterdam, New York, p. 1

132. Schönfelder, V., Bennett, K., Bloemen, H. et al., 1995, Annals of New York Acad. of Sc., vol. 759, p. 226

133. Schönfelder, V., Bloemen, H., Collmar, W., et al 2000b, in "The Fifth Compton Symp.", AIP Conf. Proc. 510, 54

134. Schönfelder, V. 1977, in Proc. of 15th Int. Cosmic Ray Conf., Plovdiv, 10, 53

135. Schönfelder, V., et al. 1993, ApJ Suppl. 86, 657

136. Schönfelder, V., 2002, AN 323, 524

137. Schönfelder, V., Hirner, A., Schneider, K., 1973, Nucl. Instr. \& Methods 107, 385

138. Schönfelder, V., Graml, F., Penningsfeld, F.-P., 1980, ApJ 240, 350

139. Schönfelder, V., Graser, U., Diehl, R., 1982, A\&A 110, 138

140. Schönfelder, V., Bennett, K., Blom, J.J., et al., 2000a, A\&AS 143, 145

141. Siegert T., Diehl R., Greiner J. et al. 2016, Nature 531, 341

142. Siegert T., Diehl R., Weinberger C. et al. 2019, A\&A 626, A73

143. Slowikowska A., Kanbach G., Kramer M., Stefanescu A., 2009, MN 397, 103

144. Smartt S.J., Chen T.-W., Jerkstrand A., et al. 2017, Nature 551, 75

145. Sreekumar, P., Bertsch, D.L., Dingus, B.L., et al. 1998, ApJ 494, 523-534

146. Stefanescu A., Kanbach G. Slowikowska A., et al. 2008, Nature 455, 503

147. Steinle, H., Bennett, K., Bloemen, H. et al., 1998, A\&A 338, 97

148. Straubmeier C., Kanbach G., Schrey F., 2001, Exp. Astron. 11, 157

149. Strong, A.W., Moskalenko, I.V., 2001, in "The Universe in Gamma-Rays", ed. V. Schönfelder, Astron. \& Astrophys. Library, Springer, p. 229

150. Strong A.W., Moskalenko I.V., 1998, ApJ 509, 212

151. Strong, A.W., Bignami, G.F., Bloemen, J.B.G.M. et al. 1982, A\&A 515, 404

152. Strong, A.W., Moskalenko, I.V., Reimer, O., 2000, ApJ 537, 763

153. Strong, A.W., Moskalenko, I.V., Reimer, O., 2004, ApJ 613, 962

154. Strong, A.W., Diehl R., Halloin H., et al. 2005, A\&A 444, 495

155. Swanenburg, B.N., Bennett, K., Bignami, G.F., et al, 1981, ApJ 243, L69

156. Trimble V., 1994, AIP Conf. Proc. 304, 40

157. Trombka, J.L., et al, 1973, ApJ 181, 737

158. Ubertini P., Lebrun F., DiCocco G. et al. 1999, Ap. Lett. \& Comm. 39, 331

159. Urry C.M., Padovani P., 1995, PASP 107, 803

160. van Dijk R., Bennett K., Collmar W. et al., 1995, A\&A 296, L33

161. Vedrenne G., Schönfelder V., Albernhe F. et al. 1999, Ap. Lett. \& Comm. 39, 325

162. Vestrand W.T., Share G.H., Murphy R.J., et al. 1999, ApJ Suppl. 120409

163. Voges W., Pinkau K., 1974, Proc. Conf. on Transient Cosmic Gamma- and X-ray Sources, ed. I.B. Strong, Los Alamos, Report LA-5505-C, p. 49

164. von Ballmoos P., Diehl R., Schönfelder, V., 1987a, ApJ 312, 134

165. von Ballmoos P., Diehl R., Schönfelder, V., 1987b, ApJ 318, 654

166. von Kienlin A., Meegan C.A., Paciesas W.S. et al. 2014, ApJS 211, 13

167. von Kienlin A., Meegan C.A., Paciesas W.S. et al. 2020, ApJ 893, 46

168. Weidenspointner, G., et al, 2000, in Proc. of 5th Compton Symp., AIP Conf. Proc. 510, 462

169. Weidenspointner, G., Harris M., Ferguson C., et al. 2010, Astrophysics Source Code Library, https://ascl.net/1010.081

170. Weidenspointner, G., et al., 2001, A\&A 368, 347

171. Weidenspointner, G., Kiener J, Gros M., et al., 2003, A\&A 411, L113

172. Weidenspointner, G., Harris M.J., Sturner S., Teegarden B.J., Ferguson C. 2005, ApJS 156, 69

173. Weidenspointner, G., Shrader C.R., Knödlseder J. et al., 2006, A\&A 450, 1013

174. Weidenspointner, G., Skinner G., Jean P., et al. 2008, Nature 451, 159

175. Yu H.-F., Greiner J., van Eerten H.J., et al. 2015a, A\&A 573, A81

176. Yu H.-F., van Eerten H.J., Greiner J., et al. 2015b, A\&A 583, A129

177. Yu H.-F., Preece R., Greiner J., et al. 2016, A\&A 588, A135

178. Zoglauer A., 2019, Astrophysics Source Code Library, https://ascl.net/1906.018

179. Zoglauer A., Andritschke R., Kanbach G., Bloser P.F., Litvinenko V.N., 2004, in Proc. 5th INTEGRAL Workshop, Feb. 2004, Munich, Eds. V. Schönfelder, G. Lichti \& C. Winkler, ESA SP-552, p. 921

180. Zoglauer A., Andritschke R., Schopper F., 2006, New Astron. Rev. 50, 629 